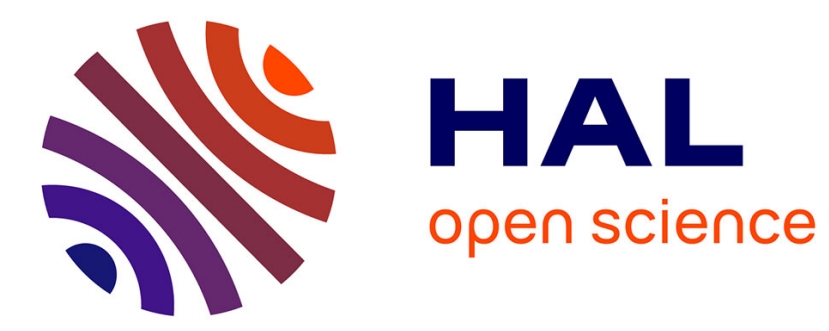

\title{
Les reliques de Lothéa (Quimperlé, Finistère) : une tombe aux connexions atlantiques entre Campaniforme et âge du Bronze ancien
}

Clément Nicolas, Pailler Yvan, Pierre Stéphan, Henri Gandois

\section{To cite this version:}

Clément Nicolas, Pailler Yvan, Pierre Stéphan, Henri Gandois. Les reliques de Lothéa (Quimperlé, Finistère): une tombe aux connexions atlantiques entre Campaniforme et âge du Bronze ancien. Gallia Préhistoire - Préhistoire de la France dans son contexte européen, 2013, 55, pp.181-227. 10.3406/galip.2013.2501 . hal-01093125

\section{HAL Id: hal-01093125 \\ https://hal.science/hal-01093125}

Submitted on 19 Dec 2019

HAL is a multi-disciplinary open access archive for the deposit and dissemination of scientific research documents, whether they are published or not. The documents may come from teaching and research institutions in France or abroad, or from public or private research centers.
L'archive ouverte pluridisciplinaire HAL, est destinée au dépôt et à la diffusion de documents scientifiques de niveau recherche, publiés ou non, émanant des établissements d'enseignement et de recherche français ou étrangers, des laboratoires publics ou privés.

\section{(ㅇ)(1) $\$$}

Distributed under a Creative Commons Attribution - NonCommercial - NoDerivatives| 4.0 


\title{
Les reliques de Lothéa (Quimperlé, Finistère) : une tombe aux connexions atlantiques entre Campaniforme et Âge du Bronze ancien
}

\author{
Clément NICOLAS *, Yvan PAILLER **, Pierre STEPHAN ${ }^{* * *}$, Henri GANDOIS ****
}

Mots-Cús. Bretagne, tumulus, mégalithisme, tombe individuelle, Néolithique final, Campaniforme, Âge du Bronze ancien, jadéitite, brassard d'archer, flèches, poignards, cuivre arsénié, chaînes de spirales, or, argent, relations atlantiques. Résumé. En 1843, Arthur Boutarel, inspecteur des forêts de Lorient, fouille un tumulus dans la forêt de Carnoët, près du hameau de Lothéa (Quimperlé, Finistère). La découverte de la relation de fouilles, restée inédite durant près de 170 ans, porte à une révision de cette tombe à la lumière des avancées de la recherche. Le tertre, par sa taille, appartient aux grands tumulus de l'Âge du Bronze ancien de Bretagne. Le caveau funéraire est construit selon les principes de l'architecture mégalithique et peut être considéré comme un lien entre les sépultures collectives du Néolithique et les tombes individuelles de l'Âge du Bronze. Le mobilier, particulièrement riche, révèle une triple composante avec des éléments attribuables au Néolithique final (pendeloque en jadéitite), au Campaniforme (brassard d'archer, flèches à pédoncule et ailerons équarris) et au tout début du Bronze ancien (poignards, hache). Trois grands poignards de type Quimperlé témoignent de relations atlantiques par leurs formes et leur alliage fait de cuivre et d'arsenic. Ces relations à longue distance sont confirmées par la présence dans la tombe de deux chaînes de spirales, une en or et une en argent, qui proviennent selon toute vraisemblance du nord-ouest de la péninsule Ibérique.
Keywords. Brittany, barrow, megalithism, individual grave, late Neolithic, Beaker, Early Bronze Age, jadeitite, wristguard, arrowheads, daggers, arsenical copper, spiraled chains, gold, silver, Atlantic connections. Aвsтract. In 1843, Arthur Boutarel, the Lorient forest inspector, excavates a barrow in Carnoët forest near the hamlet of Lothéa (Quimperlé, Finistère, France). The discovery of the excavation notes, unpublished for over near 170 years, enables this grave to be reviewed in the light of recent research. The mound, by its dimensions, belongs to the class of Breton large early Bronze Age barrows. The grave is of megalithic construction and can be considered as a link between Neolithic collective tombs and Bronze Age individual graves. The grave-goods, particularly rich, show a triple component with artefacts attributed to the Late Neolithic (jadeitite hatchet-pendant), the Beaker culture (wristguard, squared barbed-and-tanged arrowheads) and the beginnings of the Early Bronze Age (daggers, axe). Three long daggers of Quimperlé type provide evidence for Atlantic connections by their shape and alloy, consisting of copper and arsenic. These long-distance relations are confirmed by the presence in the grave of two spiraled chains, one gold and the other silver, which most probably come from north-western Iberia.

translation: Magen O'Farrell

\footnotetext{
* Doctorant, université Paris 1 - Panthéon-Sorbonne CNRS, UMR8215 Trajectoires, Maison de l'archéologie et de l'ethnologie, 21, allée de l’Université, 92023 Nanterre cedex, clement.nicolas@mae.u-paris1.fr.

** INRAP CIF, centre archéologique de Tours CNRS, UMR8215 Trajectoires, Maison de l'archéologie et de l'ethnologie, 21, allée de l'Université, 92023 Nanterre cede et Géomer - Laboratoire LETG-Brest,UMR6554 CNRS, Institut Universitaire Européen de la Mer, place Nicolas Copernic, 29280 POUZANE, yvan.pailler@inrap.fr.

*** Chargé de recherche, Laboratoire LETG-Brest,UMR6554 CNRS, Institut Universitaire Européen de la Mer, place Nicolas Copernic, 29280 POUZANE, stephan.pierre00@gmail.com.

**** Doctorant, université Paris 1 - Panthéon-Sorbonne CNRS, UMR8215 Trajectoires, Maison de l'archéologie et de l'ethnologie, 21, allée de l’Université, 92023 Nanterre cedex, henri.gandois@gmail.com.
} 


\section{The relics of Lothéa (Quimperlé, Finistère) : a grave with atlantic connections between the Bell Beaker and Early Bronze Age}

On August 14, 1843, Arthur Boutarel, the Lorient forest inspector (Magny, 1856), undertook the excavation of the Lothéa barrow, located in the forest of Carnoët (Quimperlé, Finistère) (fig. 1, 20). This excavation was part of the Romantic and Celtic movement of the late $18^{\text {th }}$ century and early $19^{\text {th }}$ century during which archaeological excavations became more frequent (Cambry, 1799; Fréminville, 1832; Mérimée, 1837; Briard, 1970b; Chaigneau, 2009). This discovery became known only through indirect accounts, in a period in which the learned societies of Brittany were in their infancy (Giot, 1997). Since this time, several generations of archaeologists have attempted to learn more about the nature of this monument and the results of its excavation (Chatellier, 1844 and 1851; Fréminville, 1845; Lukis, 1846; Taylor et al., 1846; Le Men, 1868; Audran, 1874; Micault, 1881; Bertrand, 1884 and 1891; Société d'Emulation des Côtes-du-Nord, 1884; Serret, 1885 and 1901; Chatellier, 1889 and 1907; Aveneau de la Grancière, 1899; Mortillet, 1902 and 1903; Briard, L'Helgouac'h, 1957; Briard, Mohen, 1974; Briard et al., 1977b; Eluère, 1982 and 1986; Giot et al., 1979; Briard, 2000; Balquet, 2001) (fig. 2 to 5). The present article would be just one more among many if it was not inspired by the discovery made by one of us (Y. P.) of the previously unknown account by the excavator, Arthur Boutarel, to the Mediathèque de l'Architecte et du Patrimoine in Paris $\left(\mathrm{n}^{\circ}\right.$ 0080/026/0019). This account is accompanied by a report by Prosper Mérimée, Inspector General of Historic Monuments (Bercé, 1979, p. 323) and the excavation report concerning the second barrow excavated by Arthur Boutarel at Kerivoalen (Quimperlé).

Arthur Boutarel's description of the excavations relates the exact contents of the artifacts found in the Lothéa tomb, and is corroborated by various documents (tabl. I; fig. 3). It allows a distinction to be made between the objects found in the grave and the false "relics" later mistakenly attributed to it (fig. 6). In 1844, the archaeological artifacts of Lothéa were integrated into the collections of the Cluny Museum in Paris (Revue archéologique, 1844; Sommerard, 1847 and 1883). They were later transferred to the National Archaeology Museum in Saint-Germain-en-Laye (Reinach, 1898 and 1917; Mohen et al., 1994), except for one gold chain (Henry, 2008). Several objects have since disappeared: two pieces of a weapon and one rod in a copper alloy, a silver spiral chain, seven flint arrowheads, one quartzite pebble and one copper alloy axe head, probably stolen at the time of the discovery (Le Men, 1868).

The Lothéa barrow is not alone in its environment (Le Roux, 1981; Paulet, 2005; Galliou, 2010), belonging to a small concentration of Megalithic burial mounds and cists dated to the Late Neolithic, Bell Beaker and Early Bronze Age periods (Euzenot, 1868 and 1878; Le Pontois, 1890; Giot, 1960; Le Roux, 1974, 1975 and 1999; Brunet et al., 2004; Gouézin, 2007; Galliou, 2009; Pailler et al., 2010; Donnart, 2011) (fig. 1). During the Bell Beaker period, many of the Megalithic monuments were still used. Middle Neolithic passage graves and Late/Final Neolithic gallery graves were reused (Chatellier, 1883a; L'Helgouac'h, 1970; Treinen, 1970; Briard, 1983; Salanova, 2003). The presence of sites rich in cassiterite (Guiges, 1970) and a navigable river could explain this concentration of Early Bronze Age tombs near the coastal river of Laita.

The Lothéa barrow still displays traces of its excavation in 1843 , corresponding to two large trenches, 3 to $4 \mathrm{~m}$ wide (fig. 7 to 9). The volume of the barrow, including the excavation back dirt, is $1580 \pm$ $32 \mathrm{~m}^{3}$. Its diameter must have originally been $26 \mathrm{~m}$ and its height 4 $\mathrm{m}$. The burial chamber was composed of nine edge stones in local orthogneiss (Cogné et al., 1973) (fig. 10). It is oriented east-west and measures $2.5 \mathrm{~m}$ long, $2 \mathrm{~m}$ wide and $1.45 \mathrm{~m}$ high. The slab cover, which is now broken, was $2.2 \mathrm{~m}$ long, $1.8 \mathrm{~m}$ wide and $0.45 \mathrm{~m}$ thick. A cairn surrounded and covered the tomb. It is partially visible in section and is composed of small stones measuring 15 to $20 \mathrm{~cm}$. The cairn is approximately $1.7 \mathrm{~m}$ high and its diameter at least $8 \mathrm{~m}$ - making it one of the largest in Brittany along with those of Kervini (Briard, 1972) and La Motta (Butler, Waterbolk, 1974). The rest of the mound is composed of a homogeneous layer of yellow silty-clay.

The Kerivoalen barrow is better preserved and apparently less spread out and smaller than that of Lothéa. It is $19 \mathrm{~m}$ in diameter and $1.7 \mathrm{~m}$ high (fig. 9). It is also crossed by two trenches, north-south and east-west. The excavation of this barrow revealed no internal structure and yielded very few artifacts other than a few potsherds and charcoal originating from the mound sediments.

The tomb of Lothéa yielded several objects attributable to periods from the Late Neolithic to the Early Bronze Age;

- A gold spiral chain (Eluère 1986) (fig. 11, fig. 12, 1 and fig. 13, 1)

- A silver spiral chain (fig. 12, 2)

- Four daggers in arsenical copper decorated with one to three grooves parallel to the edges (tabl. II; fig. 11, fig. 14, 1 to 4). The arsenic, whether or not it was intentionally added, probably contributed to the preservation of the metal (Hundt, 1971; Papillon, 1997; Krause, 2003; Roussot-Laroque, 2008). The very good state of preservation of the daggers allows a few technological and functional observations to be made (Quilliec, 2007) (fig. 13, 2 to 6). Three of the long blades (fig. 14, 1 to 3) are of the Quimperlé type, and the fourth one (fig. 14, 4) of the Trévérec type (Micault, 1883; Gallay, 1981; Briard, 1984; Needham, 2000a and b). The Quimperlé type is thought to be one of the oldest of the Armoricain type daggers. The arsenical copper of the Lothéa daggers confirms their old dating since this material was used in Atlantic Europe during the Bell Beaker period and the beginning of the Early Bronze Age (Briard et al., 1998; Gandois, 2008 and 2011).

- A bronze heel (tabl. II; fig. 11, fig. 14, 5). This object was probably made by the lost-wax casting method, attested in the Early Bronze Age (Schwenzer, 2009) and known elsewhere in Brittany (Aveneau de la Grancière, 1898; Needham, 2000a). It is decorated with fishbone-shaped incisions under the under the mouth and near a slight median swelling. A circle in hollow dots emphasizes the two perforations. The proportion of tin (13.2\%) in this heel appears unusually high and it is possible that this object did not originate from the tomb of Lothéa (intrusive object, mixed collections?).

- A "copper rod" (fig. 14, 6). This object could correspond to an ingot (Tinevez, 2007; Le Bihan, Villard, 2010).

- A "bronze axe" with slight ridges (fig. 14, 7). This type of axe is found in many Early Bronze Age graves in Brittany (Briard, 1984). It is nonetheless not certain that it originally came from the tomb of Lothéa (Le Men, 1868).

- Seven flint arrowheads with a pointed tang and squared barbs 
(fig. 15, 3 to 5). This type of arrowhead is found in Brittany in Bell Beaker and Early Bronze Age contexts (Le Rouzic, 1898; Chatellier, 1907; Gaillard, 1906; Le Provost et al., 1972; Le Bihan, 1993; Le Bihan et al., 1994; Le Goffic, 1996 and 1999; Annic, 2000; Balquet, 2001; Nicolas, 2011 and 2012).

- An archer's wrist-guard with four holes in crimson schist (fig. 11, fig. 15, 1). Traces of use visible near the perforations (fig. 13, 8) were probably created by the attachment or wearing of the wrist-guard (Bonnardin, 2009; Fokkens et al., 2008). The Lothéa bracer is one of the very few in Brittany that have four perforations (Le Men 1877; Briard, L'Helgouach, 1957; Sangmeister, 1964; Treinen, 1970; Riquet et al., 1963; L'Helgouac'h, 1995; Le Roux, 1999). The stone wrist-guards of the Bell Beaker period are different from those of the Early Bronze Age, which are made from precious or exotic materials (Butler, Waterbolk, 1974; Briard, 1970a; Briard et al., 1994; Needham, 2000a).

- A hatchet-pendant in jadeite from Mont-Viso in Italy (Pétrequin et al., 2012) (fig. 11, fig. 15, 2). It was shaped by pecking (fig. 13, 7). It could date to the Late Neolithic (Bailloud, 1964; Jeudy et al., 1997; Bordreuil et al., 2008) and have been re-shaped during the Bell Beaker (Chatellier, 1883b, L'Helgouac'h, 1965; Tinevez, 1988) or Early Bronze Age periods (Lisle du Dréneuc, 1892; Rogers, 1947; Briard, 1970a and b; Briard et al., 1977a; Sheridan and Pailler, 2012).

- A quartzite pebble with no use traces (Briard, Mohen, 1974).

The information obtained from the artifacts discovered at Lothéa allows its attribution to a time between the Bell Beaker and Early Bronze Age periods. It is during this time that we observed the return of individual funerary monuments ${ }^{2}$, an architectural type rarely used since the Middle Neolithic I (Fontès, 1881; Boujot and Cassen, 1992), with the exception of two possible cases attributable to the Late Neolithic (Chatellier, 1881 and 1907; Giot, Cogné, 1955, Le Rouzic, 1965). The Lothéa barrow sheds new light on the single burials of the Bell Beaker period in Brittany, which have been the subject of many studies but no syntheses. Researchers have focused more on secondary burials in the collective tombs of the Neolithic (Chatellier, 1907, p. 46-47; Briard, 1989; Giot, 1990; L'Helgouac'h, 1995; Le Roux, 1999; Salanova, 2000 and 2011). A total of fourteen graves have now been recorded in Brittany (Le Men, 1877; Chatellier, archives, 1883a, 1887 and 1897; Micault, 1883; Gaillard, 1884; Cussé, 1886; Bénard, 1929; Briard and L'Helgouac'h, 1957; Giot, 1953; L'Helgouach et al., 1970; Treinen, 1970; Le Roux, 1974 and 1975; Briard, 1978, 1984 and 1989; Briard et al., 1982; Salanova, 1996 and 2000; Boujot et al., 2000; Sparfel a,d Pailler, 2009) (tabl. III ; fig. 16 and 17). These individual tombs can be attributed to the three phases of the Bell Beaker period recently defined for northern France (Salanova, 2011). The first phase (2500-2400 B.C.) includes graves with Maritime or AOC type pottery (Lanting and Van der Waals, 1976; Lanting, 2008; Salanova, 2011), the second (2400-2150 B.C.) Epi-Maritime type beakers, and the third (2150-1950 B.C.), Epi-Bell Beaker vases. This latter phase appears to be contemporary with the first Armorican tumuli (Needham, 2000a; Nicolas et al., in press) (fig. 18).

The artifacts from Lothéa reveal numerous exchanges of objects and ideas along the Atlantic coast. The most astonishing links were found between the gold and silver chains of Lothéa and specimens from Portugal and Spain (Labet, 1859; Chatellier, 1879; Halna du
Fretay, 1889; Soeiro, 1982; Hernando Gonzalo, 1983; Briard, 1984; Eluère, 1986; Delibes de Castro, Del Val Recio, 2005) (fig. 19, 1). These spiral chains, discovered in poorly dated contexts on the Iberian Peninsula, can be linked through their distribution to a series of gold ornaments from the Bell Beaker period discovered near the Tagus estuary and Galicia, and between the Gironde estuary and the Gulf of Morbihan (Le Rouzic, 1930; Eluère, 1982; Hernando Gonzalo, 1983; Ambruster, Parreira, 1993; Salanova, 2000; Ladra et al., 2003) (fig. 20). This distribution of gold work is identical to that of other Bell Beaker artifacts (beakers, Palmela points) (Salanova, 2000; Briard, Roussot-Laroque, 2002; Prieto Martinez, Salanova, 2009). In terms of their shape and their arsenical copper composition, the long daggers of Lothéa (Quimperlé type) are very similar to Quinta de Romeira blades and confirm the existence of relations between Brittany and the north-western zone of the Iberian Peninsula (Briard, Mohen, 1974; Delibes et al., 1982; Gomez de Soto, 1990; Ambruster, Parreira, 1993; Brandherm, 2003; Krause, 2003) (tabl. II; fig. 19, 3 to 6). Other types of long arsenical copper daggers show the particularities of Atlantic metallurgy and its influence on the continental world (Piggot, 1938; Briard, 1965; Gerloff, 1975; Hundt, 1971; Almagro Gorbea, 1972; Delibes et al., 1982; Guilaine, Briois, 1984; Gomez de Soto, 1990; Needham, 2000b; Brandherm, 2003). Finally, parallels can be drawn between the archer's wristguard from Lothéa and the Iberian specimens (Sangmeister, 1964) (fig. 19, 2).

The weapons and ornaments of Lothéa are part of the numerous technical and cultural changes that occurred at the end of the $3^{\text {rd }}$ millennium B.C. along the Atlantic coast of Europe (the transition from copper to bronze metallurgy, the "fission" of the Bell Beaker culture, the emergence of Early Bronze Age cultures) (Harrison, 1974; Almagro Gorbea, 1997; Needham, 2005; Brandherm, 2007; Strahm, 2007). The absolute chronology of these evolutions remains difficult to perceive due to certain poorly dated contexts, the imprecision of the ${ }^{14} \mathrm{C}$ calibration curve, and in some cases, the lack of radiocarbon dates (Voruz, 1996). This results in a more or less reliable chronological phasing for the different cultures of the Atlantic coast (Harrison, 1988; Almagro-Gorbea, 1997; Bailly, Salanova, 1999; Hughes, 2000; Needham, 2005; Sheridan, 2007; Lanting, 2008; Bettencourt, 2010; Salanova, 2011; Nicolas et al., in press) (fig. 18). Taking into account the few radiocarbon dates available for the artifacts connected with Lothéa, we can unambiguously attribute this tomb to the end of the $3^{\text {rd }}$ millennium B.C. Given the chronology of the Bell Beaker and Early Bronze Age periods in Brittany (Needham, 2000a; Salanova, 2011; Nicolas et al., in press), we are inclined to situate the barrow of Lothéa to sometime around the $22^{\text {nd }}$ century BC.

Though numerous questions remain unresolved, the re-discovery of A. Boutarel's account of his excavations has reminded us of the historical importance of the tomb of Lothéa. This grave must have contained the remains of an important person, heir to the Bell Beaker culture and founder of the elites that would then reign over Brittany (Briard, 1984). For this period, it attests to long distance movements of ideas, objects and people, who are today increasingly perceptible through archaeology (Tomalin, 1988; Briard, 1998; Needham, Woodward, 2008; Fitzpatrick, 2009; Cook et al., 2010; Chenery, Evans, 2011; Salanova et al., 2011). 


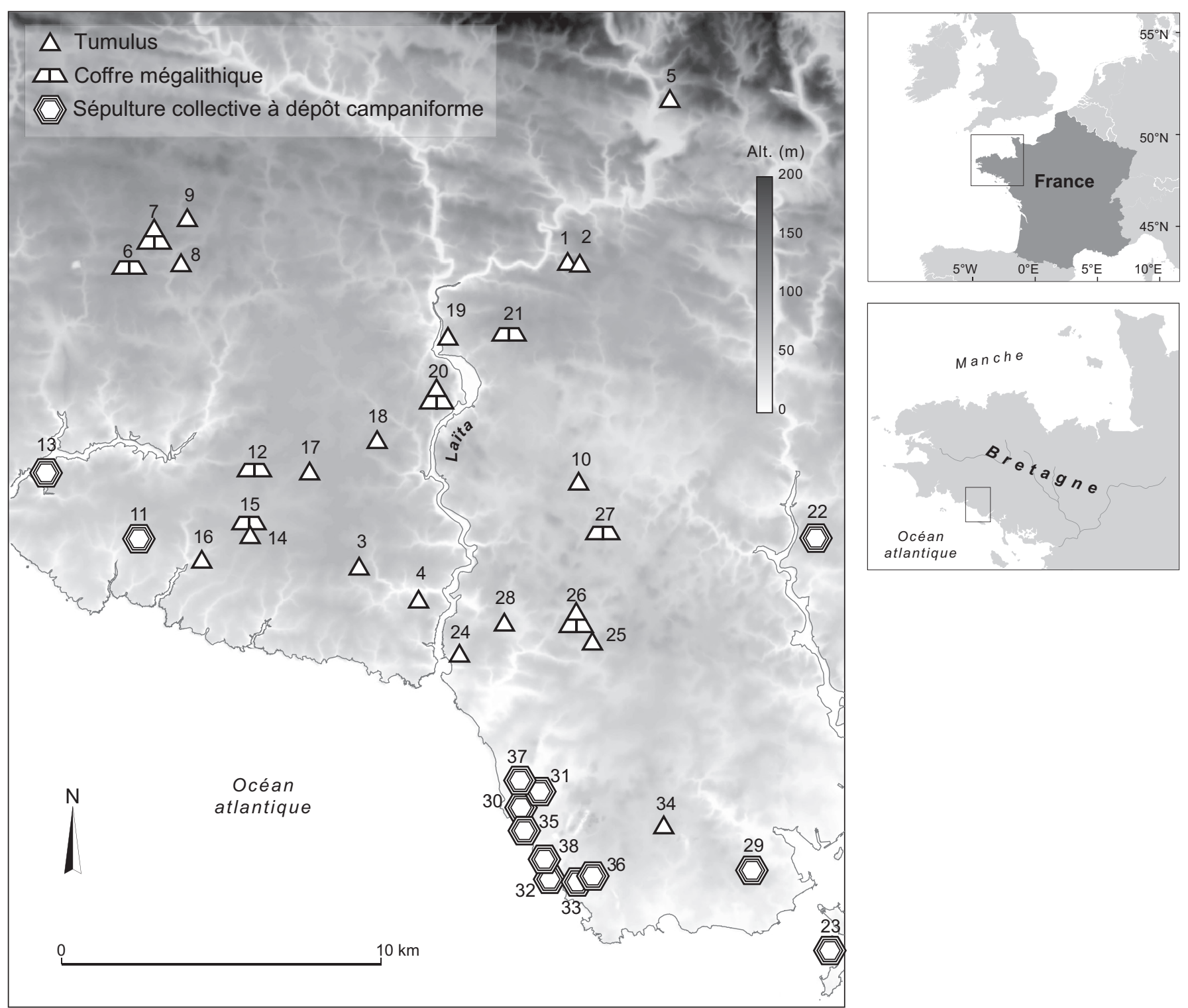

Fig. 1 - Sépultures collectives à dépôts campaniformes, tumulus et coffres mégalithiques des rives de la Laïta. Collective burials with Bell Beakers, barrows and megalithic cists around Laïta banks.

Finistère : $n^{\circ} 1$ et $2:$ Méné Béré, Arzano; $n^{\circ} 3:$ Kerloaz, Clohars-Carnoët; $n^{\circ} 4:$ Kervénou-Pouldu, Clohars-Carnoët; $n^{\circ} 5:$ Kervichel, Guilligomarc'h; $n^{\circ} 6$ : Goalichot, Le Trévoux; $n^{\circ} 7:$ Kerboulou, Le Trévoux ; $n^{\circ} 8:$ Laniscar, Le Trévoux; $n^{\circ} 9:$ Reunial, Le Trévoux ; $n^{\circ} 10$ : Ristinic, Locunolé ; $n^{\circ} 11$ : allée couverte de Kerandrèze, Moëlan-sur-Mer; $n^{\circ} 12:$ Kercadoret, Moëlan-sur-Mer; $n^{\circ} 13:$ allée couverte de Kermeur-Bihan, Moëlan-sur-Mer; $n^{\text {os }} 14$ et 15 : Mescleo, Moëlan-sur-Mer; $n^{\circ} 16$ : Penanprat, Moëlan-sur-Mer; $n^{\circ} 17$ : Quillimar, Moëlan-surMer; $n^{\circ} 18$ : Kerivoalen, Quimperlé; $n^{\circ} 19$ : Kerprivec, Quimperlé ; $n^{\circ} 20$ : Lothéa, Quimperlé ; $n^{\circ} 21$ : Roscasquen, Quimperlé ; Morbihan : $n^{\circ} 22$ : tombe à couloir de Nelhouët, Caudan; $n^{\circ} 23$ : sépulture à entrée latérale du Goërem, Gâvres; $n^{\circ} 24:$ Cruguel, Guidel; $n^{\circ} 25$ : Kermené, Guidel ; $n^{\circ} 26$ : Kerouarc'h, Guidel ; $n^{\circ} 27$ : Lez-Variel, Guidel; $n^{\circ} 28$ : Saint-Fiacre, Guidel; $n^{\circ} 29$ : dolmen de Kercavès, Ploemeur ; $n^{\circ} 30$ : dolmen de Beg er Lann, Ploemeur; $n^{\circ} 31$ : sépulture transeptée de Cruguellic, Ploemeur; $n^{\circ} 32$ : sépulture à entrée latérale (?) de Tuchen-Gouc'h, Ploemeur ; $n^{\circ} 33$ : tombe à couloir de Kouregan, Ploemeur ; $n^{\circ} 34$ : La Motte à Madame, Ploemeur; $n^{\circ} 35$ : dolmen de PortFétih, Ploemeur ; $n^{\circ} 36$ : dolmen de Roch-er-Penner, Ploemeur ; $n^{\circ} 37$ : tombe à couloir et chambres compartimentées de Saint-Adrien, Ploemeur ; $n^{\circ} 38$ : tertre tumulaire de Tuchen-er-Hroëk, Ploemeur. [Inventaire d'après Briard, 1984a, Salanova, 2000, Gouézin, 2007, Sparfel et Pailler, 2011] (Carte P. Stephan, CNRS ; Map P. Stephan, CNRS).

Le 14 août 1843, Arthur Boutarel, inspecteur des forêts de Lorient, entreprend la fouille du tumulus de Lothéa situé dans la forêt de Carnoët (Quimperlé, Finistère ; fig. 1, n 20). Cette exploration, une des toutes premières du genre en Bretagne, eut rapidement un retentissement à l'échelle nationale. Néanmoins seuls des comptes-rendus indirects firent état de cette découverte, à une époque où les sociétés savantes en Bretagne en étaient à leurs balbutiements (Giot, 1997, p. 36). Depuis lors, plusieurs générations d'archéologues ont cherché à préciser la nature de ce monument et le résultat de la fouille. Le présent article ne serait qu'une énième publication s'il n'était pas motivé par la découverte de la relation inédite du fouilleur, Arthur Boutarel, à la Médiathèque de l'Architecte et du Patrimoine à Paris (n 0080/026/0019) par l'un de nous (Y. P.). Cette 
relation de fouilles est peut-être tombée dans l'oubli en raison d'une erreur d'enregistrement, étant inscrite par homonymie dans le dossier de la commune de Carnoët (Côtes-d'Armor). Ce compte-rendu donne le contenu exact du mobilier trouvé dans la tombe de Lothéa, corroboré par différents documents. Il nous porte à entreprendre une révision du mobilier de ce tumulus couplée avec une nouvelle étude du monument. Le contenu exceptionnel de la tombe permet de reconsidérer certains aspects des relations entre le Campaniforme et l'Âge du Bronze ancien en Bretagne et plus largement sur les circulations d'objets et d'idées le long de la façade atlantique.

\section{LA CORRESPONDANCE}

La relation de fouilles du tumulus de Lothéa est contenue dans une correspondance de quatorze lettres et un rapport écrits entre le 19 août 1843 et le 16 mai 1845 entre les acteurs de différentes administrations : les ministères des Finances et de l'Intérieur, respectivement tutelles de l'administration des Forêts et de la direction des Beaux-arts, et la commission des Monuments historiques. Les auteurs de ces courriers sont Arthur Boutarel, inspecteur des Forêts à Lorient, Victorin Legrand, directeur général de l'administration des Forêts, Jean Lacave-Laplagne, ministre des Finances, le comte Tanneguy Duchâtel, ministre de l'Intérieur, Hygen-Auguste Cavé, directeur des Beaux-Arts et Prosper Mérimée, inspecteur général des Monuments historiques. Ces courriers, passant par la voie hiérarchique, sont assez répétitifs et présentent peu d'intérêt, si ce n'est celui d'illustrer les rouages administratifs sous la monarchie de Juillet (1830-1848).

Cet échange épistolaire a pour but essentiellement de trouver une destination au mobilier découvert à Lothéa (le musée de Cluny, en l'occurrence), puis le dédommagement des fouilles de Lothéa, estimé à 112 francs par Arthur Boutarel (lettre du 19 décembre 1843), et une subvention de 388 francs pour que ce dernier puisse ouvrir un second tumulus dans la forêt de Carnoët. À cette occasion, la commission des Monument historiques transmet, sur proposition de Prosper Mérimée, le dossier d'Arthur Boutarel à la sous-commission des Médailles et Mentions honorables (Bercé, 1979, p. 323). Arthur Boutarel reçoit une médaille le 6 décembre 1844.

Nous reproduisons ici les relations de fouilles des deux tumulus de la forêt de Carnoët par Arthur Boutarel ainsi que le rapport de Prosper Mérimée à la commission des Monuments historiques, comme témoignage de l'encouragement de l'État aux recherches archéologiques. À travers le suivi de l'opération par les autorités, le dépôt du mobilier dans les collections d'un musée national et la subvention des fouilles, on peut considérer la fouille de Lothéa comme la première fouille programmée de l'histoire de l'archéologie de Bretagne.

\section{5ème conservation \\ Inspection de Lorient \\ Département du Finistère}

Administration des Forêts

L'an 1843 , le 19 du mois d'août.

Je soussigné Jean Antoine Arthur Boutarel Inspecteur des forêts à la résidence de Lorient, fais observer que dans la forêt royale de Carnoët, située sur les territoires de la commune de Quimperlé, département du Finistère, il existe un plateau au milieu duquel se trouve un monticule. Ayant souvent remarqué que ce monticule, qui a à sa base quarante mètres de diamètre, et qui a une élévation de six mètres environ au-dessus du niveau du sol, n'était composé que de terres rapportées, j'ai pensé qu'il devait renfermer un tombeau et j'ai pris le parti d'y faire exécuter des fouilles qui ont été commencées, en ma présence, le 14 du courant et continuées le lendemain et le surlendemain. J'ai tracé deux lignes de deux mètres de largeur, perpendiculaires entre elles, et se croisant au sommet du monticule. J'ai fait enlever par mes ouvriers la terre qui se trouvait dans les lignes ainsi tracées et j'ai ensuite trouvé une grande quantité de moëllons placés pêle-mêle les uns sur les autres sans former la moindre maçonnerie. J'ai fait enlever tous ces moëllons et j'ai trouvé une grande pierre brute de granit, qui avait environ deux mètres vingt centimètres de longueur, sur un mètre quatre vingt centimètres de largeur, et quarante cinq centimètres d'épaisseur. Cette grande pierre était placée horizontalement et supportée par d'autres grandes pierres brutes placées de champs, et formant entre elles le tombeau que je cherchais. Toutes ces pierres étaient jointes par un mastic qui était gras et mou, et qui s'est extraordinairement durci, quand il a été au contact de l'air et du soleil.

Pour enlever la grande pierre qui couvrait le tombeau, les ouvriers que j'ai employés quoiqu'aidés de Mr. le Garde Général Busigny, des deux gardes de la dite forêt et d'une foule de curieux, ont fait d'inutiles efforts. J'ai donc été obligé de prendre le parti de faire casser cette énorme pierre par des maçons qui en ont immédiatement rompu un gros morceau, lequel morceau une fois enlevé, m’a permis de distinguer confusément des glaives ou des lances et d'autres objets. Aussitôt j'ai laissé descendre dans le tombeau une personne de confiance qui m'a remis sur le champ

$1^{\circ}$. une chaine en or très fin du poids de 225 grammes, composé de six grands anneaux ayant chacun quatre tours, (cette chaine est très bien conservée)

$2^{\circ}$. une chaine en argent fortement oxydée, et composée d'un grand anneau et de deux autres beaucoup moins grands ;

$3^{\circ}$. un casse-tête qui n'est autre chose qu'un gros caillou ayant la forme d'une fève

$4^{\circ}$. trois instruments tranchants oxydés que bien des personnes considèrent comme des glaives et d'autres comme des lances ; 
$5^{\circ}$. une pique oxydée ;

$6^{\circ}$. un petit poignard oxydé ;

$7^{\circ}$. deux morceaux de métal oxydé, formant probablement les débris de quelques armes.

Les lances ou glaives et la pique, sont, dit-on en argent renfermant beaucoup d'alliage. On voit encore bien distinctement sur un des glaives une dorure qui annonce que cette arme était d'une grande beauté. Pour pouvoir donner de plus amples renseignements il faudrait que j'eusse fait nettoyer tous les objets que j'ai trouvés ; mais je n'ai pas cru devoir m'occuper d'une pareille opération, dans la crainte de rendre les objets moins précieux et moins curieux.

Quant au petit poignard, il a été enlevé des mains de Mr. le Garde Général par un curieux qui a demandé à le voir, et qui ne l'a plus rendu. Il peut cependant se faire que ce poignard qui a passé dans plusieurs mains ait été abandonné par les curieux pressés d'examiner les autres objets et qu'il se soit ainsi égaré. En conséquence s'il n'a pas été soustrait frauduleusement, je conserve encore l'espoir de le retrouver.

On a ensuite continué à casser un morceau [de] l'énorme pierre tumulaire, et quand cette opération a été terminée, j’ai reconnu que ce tombeau renfermait à environ 80 centimètres de profondeur une terre également unie sur laquelle avaient été pris tous les objets précités.

J'ai alors fait extraire du tombeau toute cette terre dans laquelle la pelle s'enfonçait facilement, et j'ai visité attentivement chacune des pellées desquelles j'ai retiré,

$1^{\circ}$. une jolie pierre rouge qui a la forme d'un quadrilatère rectangle. Elle a un trou au sommet de chaque angle. Elle est probablement une ancienne fronde.

$2^{\circ}$. une amulette en pierre verte taillée, ayant la forme d'un trapèze dont les deux bases parallèles sont arrondies. Cette amulette a un trou très régulièrement fait.

$3^{\circ} .7$ flèches en silex, et dentelées, qui annoncent que le tombeau dont il s'agit, date d'une époque très reculée, et qu'il est même antérieur à la fondation de la monarchie.

J'ai en outre retiré d'un joint des deux grandes pierres placées de champ, qui forment le tombeau, une petite baguette en cuivre et plusieurs morceaux du mastic dont j'ai parlé plus haut.

La fouille que j'ai fait faire, a duré trois jours, et chaque nuit, les deux gardes Lemour et Croissant ont fait faction auprès du tombeau, pour que des malfaiteurs ne vinssent pour faire des fouilles frauduleuses.

En fouillant je n'ai trouvé ni ossements, ni urnes, ni les poignées des glaives et du poignard, ni le manche du casse-tête, ni les bois de la pique et des flèches. Il parait que le temps a tout détruit et que tout est devenu terre, hors les objets trouvés.

Boutarel

Ministère de l'Intérieur

Direction des Beaux-Arts

Commission des Monuments historiques

Rapport à la Commission

Par Mr Mérimée

sur les fouilles de Carnouët (Finistère)

Séance du 14 Juin 1844

Mr le Préfet du Finistère réclame pour Mr Boutarel, le remboursement d'une somme de $112 \mathrm{f}$. pour fouilles exécutés par lui dans la forêt de Carnoët. Mr. Boutarel ne fournit point de mémoire, et ne fait d'ailleurs aucune réclamation. On a rapporté que la valeur intrinsèque des objets d'or et d'argent assignés au Mère de l'Intérieur par Mr. Boutarel s'élève à plus de $1200 \mathrm{f}$.

Mr. Boutarel signale l'existence d'un tumulus voisin de celui qui a produit la découverte des objets déposés actuellement au musée de l'hôtel de Cluny et estime à $400 \mathrm{f}$ la dépense que des fouilles occasionneraient.

Le rapporteur propose d'allouer une somme de $500 \mathrm{f}$. sur l'exercice de $1844 \mathrm{à} \mathrm{Mr}$. Boutarel, tant pour l'indemnité de laquelle il a droit, que pour la continuation des fouilles dans la forêt de Carnoët.

La Commission jugera peut-être à propos de renvoyer en même temps le dossier de Mr. Boutarel à la S[ous-]Commission des Médailles et mentions honorables.

P. Mérimée 
A son Excellence Monsieur le Ministre de l'Intérieur

Monsieur le Ministre,

Par la lettre que vous m'avez fait l'honneur de m'écrire le 13 juillet dernier, vous m'avez annoncé que, sur l'avis de la Commission des monuments historiques, vous veniez d'allouer une somme de 500 francs pour la continuation des fouilles pratiquées dans la forêt domaniale de Carnoët (Finistère) et vous m'avez invité à en diriger les travaux.

En Septembre 1844, j'ai donc fait ouvrir dans la forêt précitée, un monticule ayant les mêmes formes extérieures que celui ouvert en 1843 ; mais je n'ai pas eu le même bonheur que l'année précédente : l'ouverture de ce monticule par deux grandes et profondes tranchées perpendiculaires entre elles, m'a démontré qu'il ne se composait que de terres rapportées ne renfermant pas le moindre caillou, et dans lesquelles je n'ai trouvé aucun monument, aucun objet d'antiquité... absolument rien que quelques légers débris de poterie, qui tombaient en poussière lorsqu'on les touchait, et quelques petits morceaux de charbons de bois. Je conclus de là que les corps de plusieurs centaines de soldats tués dans un combat, ont été brûlés, que leurs cendres ont été placées dans les urnes ; et que ces urnes ont ensuite été recouvertes d'une énorme quantité de terres. Voilà, à mon avis, ce qui a formé le monticule dont il s'agit, qui était peuplé de brins de chêne très serrés âgés d'environ 15 ans, et ayant évidemment succédé à plusieurs générations d'arbres ; car en extrayant la terre que j'ai trouvé plusieurs vieilles souches en partie pourries ; lesquelles souches sont assurément celles des arbres abattus dans ces exploitations anciennement faites.

Après avoir fini de fouiller infructueusement ce monticule, je me suis transporté, avec tous mes ouvriers, sur celui dans lequel j’ai découvert, en 1843, un tombeau où se trouvaient des armes celtiques et la chaine en or qui sont aujourd'hui au musée des Thermes et de l'hôtel de Cluny.

Arrivé là, j'y ai fait continuer les fouilles en faisant enlever toutes les pierres qui entouraient ce tombeau, j'en ai trouvé une en granit, taillées en linteau présentant d'un côté, une surface entièrement plane, et de l'autre une surface convexe qui se termine de manière à former avec la partie plate de cette pierre qui n'est plus entière, un tranchant assez aigu, mais légèrement émoussé.

J'ai cette pierre qui était évidemment une arme de la même époque que celles déjà déposées au musée. Il y a une échancrure artistement taillée, où l'on adaptait probablement un manche .

J'ai en outre trouvé au pied du tombeau précité plusieurs morceaux d'un mastic noir et mou, qui n'en forment plus aujourd'hui qu'une [?] oul de forme à peu près sphérique ; attendu qu'en les pressant avec les mains, ils se sont liés ensemble. Ce mastic, au contact de l'air, est devenu presque immédiatement d'une grande dureté.

Le tombeau dont il s'agit, est aujourd'hui complètement dégagé ; et l'accès en est on ne peut plus facile. Les voyageurs qui passent à Quimperlé, et qui en entendent parler comme d'un monument de la plus haute antiquité, viennent généralement le visiter, tant il excite leur curiosité !

Il y avait en outre dans la forêt domaniale de Carnoët une énorme pierre plate et brute, qui était couchée sur le sol. Comme toutes les personnes qui connaissent la localité présument qu'en fouillant au dessous de cette pierre, on pourrait trouver des objets d'antiquités, j'y ai fait exécuter des fouilles qui malheureusement ont été infructueuses, et qui ont été terminées en décembre dernier.

Voilà, Monsieur le Ministre, ce résultat de la continuation des fouilles, que vous avez prescrite. Je vous prie donc d'avoir la bonté de me faire savoir, si je dois vous adresser le mastic et l'arme en pierre précités; et par quelle voie il convient que je les fasse parvenir à votre Excellence.

[Boutarel]

\section{HISTORIQUE DES RECHERCHES}

\section{AVANT 1843}

Arthur Boutarel est né en 1802 de l'union de GilbertHugues Boutarel, fils du général Boutarel, et MarieAngélique de Chirol de la Brousse. Sa vocation lui vient probablement de son père qui fut capitaine d'artillerie avant de devenir inspecteur des Forêts (Magny, 1856, p. 268). On ne lui connaît pas d'intérêt particulier pour l'archéologie et les deux tumulus de la forêt de Carnoët sont à notre connaissance les deux seuls monuments qu'il ait fouillés. L'exploration du tumulus de Lothéa s'inscrit dans le mouvement romantique et celtique de la fin du XVIII ${ }^{\mathrm{e}}$ siècle et du début du XIX ${ }^{\mathrm{e}}$ siècle qui voit le déve- loppement des fouilles archéologiques (Chaigneau, 2009). Dans son Voyage dans le Finistère, Jacques Cambry (1799) signale dans les cantons du district de Quimperlé des " monuments druidiques ». Une trentaine d'années plus tard, ChristophePaulin de La Poix, dit Chevalier de Fréminville, raconte la fouille faite près de la commune de Plouvorn (Finistère) d'un « sarcophage de pierre » par le Comte de la Fruglaye : « ce tombeau renfermait parmi des cendres et des charbons, un fer de lance en bronze long de quatre pouces, la lame d'un poignard en airain, longue de dix pouces, et enfin une pointe de flèche en silex » (Fréminville, 1832, p. 66) - cette tombe put être ultérieurement attribuée à l'Âge du Bronze ancien par Jacques Briard (1970b). Le Chevalier de Fréminville précise que « les premières, les plus anciennes sépultures (et ce sont naturellement les plus simples), furent ces buttes de terre plus ou moins élevées en cône que 
l'on connaît sous le nom de tombelles (tumuli) » (Fréminville, 1832, p. 66-67). Enfin on citera la tournée dans l'ouest de la France faite par Prosper Mérimée où il décrit pour le Finistère des architectures du Moyen-Âge, des antiquités et quelques dolmens (Mérimée, 1837). Ces descriptions anciennes montrent que localement on avait parfaitement conscience de l'existence des monuments de la Préhistoire, et plus particulièrement des tumulus de l'Âge du Bronze. Ayant en tête ces découvertes ou par intuition, Arthur Boutarel pouvait prédire du tumulus de Lothéa « que ce monticule [...] n'était composé que de terres rapportées, j'ai pensé qu'il devait renfermer un tombeau » (Lettre du 19 août 1843).

\section{DES FOUILLES DE CARNOËT À AUJOURD'HUI}

Dès le 26 septembre 1844, l'archéologue britannique J. W. Lukis réalise un premier plan de la tombe de Lothéa et mesure le tumulus qui fait alors 92 pieds de diamètre, soit $28 \mathrm{~m}$ (fig. 2). La même année, Armand du Chatellier, célèbre historien et archéologue, fait une brève relation de la fouille du tumulus de Lothéa, félicitant Arthur Boutarel de sa découverte, lui adressant toutefois quelques critiques : «Malheureusement, rien ne put être dessiné sur place, et nous avons eu surtout le regret de ne pouvoir constater d'une manière sévère comment ces objets étaient placés entre eux » (Chatellier, 1844, p. 230). En 1845, Arthur Boutarel fouille le second tumulus de la forêt de Carnoët, mais en vain : « je n'ai trouvé aucun monument, aucun objet d'antiquité »(Lettre du 18 avril 1845). Cette seconde fouille ne fit pas grand bruit, mais le tumulus est signalé plus tard par René-François Le Men (1868, p. 368) sur information de Jean Audran. A la même époque, le Chevalier de Fréminville signale la fouille du tumulus de Lothéa dans son guide touristique du Finistère (Fréminville, 1845, p. 9-10) et J. W. Lukis en informe ses collègues d'outre-Manche mais avec un inventaire du mobilier multipliant les erreurs et les doublons (Lukis, 1846). En 1846, le baron Isidore Taylor publie avec le concours de Charles Nodier et Alphonse de Cailleux deux volumes concernant la Bretagne des Voyages pittoresques et romantiques dans l'ancienne France (1846). Ce précieux ouvrage renferme une lithographie réalisée par A. Guillaumot de la "fouille faite dans le tumulus de Carnouët ", où se trouve l'illustration de divers objets trouvés dans la tombe, certains étant aujourd'hui disparus (tabl. I et fig. 3). C'est par la voie de la commission des Monuments historiques, où le baron Taylor siégeait, que celui-ci a eu vent de la découverte (Bercé, 1979, p. 322). En 1851, Armand du Chatellier formule une courte diatribe à propos du dépôt des objets de Lothéa au musée de Cluny : « Voyez le fameux monument de Carnoët, près de Quimperlé, que nous fouillions nous-mêmes en 1841 ou 1842. C'était aussi d'abord un tumulus ; - puis on fouille, et l'on trouve un beau dolmen, et dans la chambre à laquelle on parvient difficilement, des armes, des bois des flèches et des joyaux en or, qu'on nous a impitoyablement enlevés pour récréer le bon Parisien quand il veut bien aller au musée Du Sommerard [le musée de Cluny]. » (Chatellier, 1851, p. 58).

Il s'écoule une quinzaine d'années avant que René-François Le Men réalise une enquête après avoir découvert les objets de Lothéa au musée de Cluny, tout en regrettant la sommaire description du mobilier (Le Men, 1868). Pour cette raison, il part à la recherche de la « foule de curieux » (Arthur Boutarel, lettre du 19 août 1843) qui assistèrent à la fouille. Il retrouve «MM. Peyron, négociant; Julien, receveur des douanes ; Binet, professeur de médecine vétérinaire à l'École d'agriculture du Lezardeau, demeurant tous trois à Quimperlé, et Le Doze, propriétaire cultivateur au manoir de l'Ile, en la commune de Clohars-Carnoët. » (Le Men, 1868, p. 365). L'enquête donne des informations complémentaires, mais certaines divergent déjà avec la relation d'Arthur Boutarel. La fouille du tumulus de Lothéa aurait été faite à l'instigation de Peyron, adjudicataire d'une coupe de bois dans la forêt de Carnoët. Ce dernier en aurait demandé l'autorisation à Arthur Boutarel, qui aurait accepté à condition que ces fouilles se fassent en sa présence. Il s'agit d'une première différence avec le récit d'Arthur Boutarel, pour qui les fouilles ont été faites à son initiative (Lettre du 19 août 1843). Les témoins rapportent la scène de l'ouverture de la tombe non sans moquerie : «M. Boutarel voulut à son tour rentrer dans la grotte, mais son embonpoint l'en ayant empêché il fit aussitôt briser la plate-forme par un maçon. » (Le Men, 1868, p. 366). La version d'Arthur Boutarel est tout autre, relatant que briser la grande dalle de couverture était le seul moyen d'accéder à la tombe (Lettre du 19 août 1843). La description du mobilier trouvé dans la tombe concorde en partie avec celle d'Arthur Boutarel (tabl. I). Cependant il y a des désaccords sur le nombre de ces objets. Les " trois instruments tranchants oxydés » et le «petit poignard oxydé » (tabl. I) deviennent « cinq poignards ou lance en bronze » et les « 7 flèches en silex » sont « au nombre de dix à douze suivant les uns, d'une vingtaine selon les autres » (Le Men, 1868, p. 366-367). Enfin René-François Le Men indique que c'est le duc de Nemours qui, de passage à Lorient, a récupéré les fruits de la fouille et les a déposés au musée de Cluny. Cette information ne paraît pas concorder avec une lettre du 8 décembre 1843 du ministère de l'Intérieur qui annonce l'envoi du chef du bureau de la direction des Beaux-arts pour récupérer les objets. Une seule donnée semble compléter la relation de fouilles de 1843 : «L'ouvrier remit ces objets [une chaîne en or, une chaîne en argent, et des poignards] à M. Boutarel et sortit de la chambre, où il fut remplacé par M. Le Doze, qui y trouva une hache en bronze que le premier explorateur n'avait pas remarquée. Mécontent de ce que M. Boutarel s'emparait de tous les objets, M. Le Doze, au lieu de lui remettre la hache qu'il venait de découvrir, la cacha dans une de ses bottes avant de sortir de la chambre. Il la donna plus tard à M. Lagillardaie, collectionneur à Quimperlé, dont le cabinet a été dispersé après sa mort. J'ai pu m'assurer, d'après le dessin que $M$. Le Doze a bien voulu me faire de cette arme, que c'était une hache à ailerons, sans anneau, semblable à celles que l'on trouve assez fréquemment en Bretagne, associées aux lances, aux épées et aux poignards en bronze ». (Le Men, 1868, p. 366). Cette « hache en bronze » semble bien correspondre à celle publiée dans la planche des Voyages pittoresques et romantiques dans l'ancienne France (Taylor et al., 1846), où l'on peut distinguer une hache à légers rebords, type récurrent dans les tumulus de l'Âge du Bronze ancien en Bretagne (Briard, 1984).

Par la suite, l'enquête de René-François Le Men (1868) fait autorité pour la fouille du tumulus de Lothéa et sera reprise par les différents chercheurs qui se sont 
intéressés au monument et à son contenu (Audran, 1874 ; Micault, 1881 ; Chatellier, 1889 et 1907 ; Aveneau de la Grancière, 1899 ; Briard, Mohen, 1974 ; Balquet, 2001). En 1881, Victor Micault précise que deux objets, qui jusqu'ici n'avaient jamais été mentionnés, proviendraient de la tombe de Lothéa : " un bracelet et une chaîne en bronze, qui remis d'abord à M. de La Gillardaie furent légués par lui à $\mathrm{M}$. le conseiller Fornier qui les possède encore dans sa belle collection » (Micault, 1881, p. 125). Ces deux éléments de parure furent publiés avec les poignards «en bronze » et les deux chaînes en or et en argent par la Société d'Emulation des Côtesdu-Nord (1884) dans les Trésors archéologiques de l'Armorique occidentale. Victor Micault semble plus au courant du nombre des pointes de flèches, il en cite « 7 au moins » (Micault, 1881). Les Trésors archéologiques de l'Armorique occidentale citent " un certain nombre de pointes de flèches en silex, au nombre de six ou sept " (Société d'Emulation des Côtes-du-Nord, 1884). La même année, M. de Sailly, inspecteur des Eaux et Forêts, dresse un plan et une vue du tumulus de Lothéa (fig. 4). Alexandre Bertrand, premier directeur du musée d'Archéologie nationale, critique les conditions de la fouille : « Ce tumulus n'avait malheureusement pas été fouillé scientifiquement. » (Bertrand, 1884, p. 106). Dans la réédition de son ouvrage $L a$ Gaule avant les Gaulois, il publie les dessins d'une flèche et de trois poignards du tumulus de Lothéa (Bertrand, 1891, fig. 124 et 128). A l'occasion de deux synthèses sur l'or et l'argent aux temps préhistoriques en France et en Europe, Adrien de Mortillet publie les dessins des deux chaînes de Lothéa (Mortillet, 1902, fig. 17 et 1903, fig. 6). Le dessin de la chaîne en or est indubitablement recopié des Trésors archéologiques de l'Armorique occidentale (Société d'Emulation des Côtesdu-Nord, 1884). En revanche, le dessin de la chaîne en argent semble être un original.

Le 14 juillet 1957, Jacques Briard et Jean L'Helgouach, son « compagnon de route scientifique » (Briard, 2000, p. 332), se déplacent en famille sur le tumulus de Lothéa pour réaliser un nouveau plan du coffre (fig. 5). Les deux jeunes archéologues, entrés récemment au Centre national de la recherche scientifique, travaillent alors à leur synthèse sur le Néolithique et les débuts de l'Âge du Bronze en Bretagne (Briard, L'Helgouach, 1957). En 1974, Jacques Briard et Jean-Pierre Mohen (1974) produisent une nouvelle publication du site comprenant l'étude du monument et du mobilier ainsi que des analyses de la composition métallique de certains objets. Ils établissent des liens avec différentes régions d'Europe (la péninsule ibérique, les îles Britanniques et l'Europe continentale). La description du dépôt funéraire de Lothéa est basée sur les observations de René-François Le Men (1868) et des Trésors archéologiques de l'Armorique occidentale (Société d'Emulation des Côtesdu-Nord, 1884). Les auteurs retrouvent une flèche supposée provenir de Carnoët, mentionnée au catalogue du musée de Quimper (Serret, 1885) et aujourd'hui conservée au musée de Préhistoire finistérienne à Penmarc'h (Briard, Mohen, 1974, p. 47). À l'époque, Jacques Briard ne semble pas connaître la planche publiée par Isidore Taylor, Charles Nodier et Alphonse de Cailleux (1846). Il rattrapera cette inadvertance dans son inventaire des Tumulus d'Armorique (Briard, 1984). Enfin, Christiane Eluère (1986) publie une nouvelle étude de la chaîne en or.

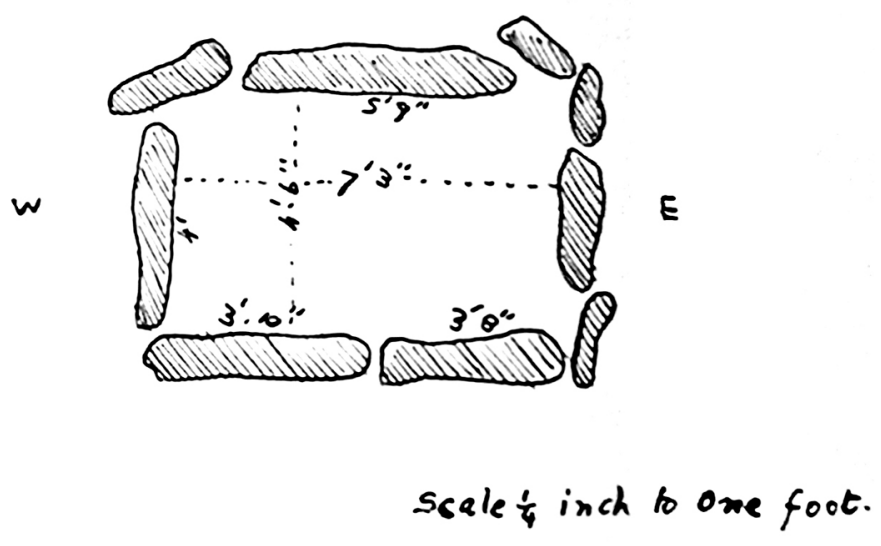

Fig. 2 - "Chambered tumulus in the Forest of Carnoët near Quimperlé, Finistère, explored aug. 14, 1843 ", plan réalisé par J. W. Lukis, le 26 septembre 1844 (archives du laboratoire Archéosciences - UMR 6566/CreAAH).

"Chambered tumulus in the Forest of Carnoët near Quimperlé, Finistère, explored aug. 14, 1843", plan made by J. W. Lukis, the September $26^{\text {th }}$ 1844 (laboratoire Archéosciences - UMR 6566/CreAAH archives).

\section{LES VRAIES, LA POSSIBLE ET LES FAUSSES RELIQUES DE LOTHÉA}

Les reliques de Lothéa, c'est d'abord au sens latin reliquia, « ce qui reste » du dépôt funéraire, car certains objets ont depuis disparu. À l'image des reliques de saints, le mobilier du défunt inhumé à Lothéa a suscité un vif intérêt de la part de chaque génération d'archéologues, encouragé par l'exceptionnalité du mobilier et les circonstances de la découverte. C'est probablement cette curiosité qui a porté certains archéologues à rechercher de possibles objets manquants, sans toute la prudence requise, créant ainsi de fausses reliques. À la lumière de la relation de fouilles d'Arthur Boutarel, il est aujourd'hui possible de les distinguer des vraies reliques provenant de Lothéa.

Cinq mois après les fouilles de Carnoët, le ministère de l'Intérieur accuse réception des objets de Lothéa (Lettre du 22 décembre 1843) après avoir envoyé le chef du bureau de la direction des Beaux-arts récupérer ces derniers (Lettre du 8 décembre 1843). Ceux-ci sont inscrits à l'inventaire du musée national du Moyen-Âge de Cluny en février 1844 (tabl. I). Une notice publiée dans le premier volume de la Revue archéologique (1844) fait mention du don au musée de Cluny avec une illustration de deux poignards. Le mobilier de Lothéa restera à Cluny pendant 43 ans sous la dénomination de « série d'objets d'origine gallo-romaine et celtique, trouvés dans un tombeau de la forêt de Carnoët (Finistère), par M. l'ingénieur Boutarel, et donnés au Musée par M. le ministre des finances » (Sommerard, 1847, p. 232, 1883, p. 619). La description qui est donnée du mobilier correspond exactement au récit de Boutarel (Lettre du 19 août 1843), reprenant certaines de ses tournures de phrase (tabl. I). Le mobilier recevra successivement trois numéros d'inventaire 1441 à 1449, puis 1798 et finalement 7659 à 7669 . Arrivé à la tête du musée de Cluny en 1885 , Alfred Darcel entreprend assez rapidement un réaménagement des collections autour du Moyen-Âge et de la Renaissance, transférant les collections d'objets préhistoriques au musée 
Tabl. I - Comparaisons entre les différents inventaires du mobilier du tumulus de Lothéa (Quimperlé, Finistère).

Comparisons between the different inventories of grave goods from the Lothéa barrow (Quimperlé, Finistère).

\begin{tabular}{|c|c|c|c|c|c|c|}
\hline Boutarel, 1843 & $\begin{array}{l}\text { Inventaire du Musée } \\
\text { de Cluny, février } \\
1844\end{array}$ & Taylor et al., 1846 & Le Men, 1868 & $\begin{array}{l}\text { Micault, } 1881 \text { et } \\
\text { Société d'Émulation } \\
\text { des Cotes-du-Nord, } \\
1884\end{array}$ & $\begin{array}{l}\text { Inventaire du Musée } \\
\text { d'Archéologie } \\
\text { Nationale, } 1887\end{array}$ & $\begin{array}{l}\text { Briard et Mohen, } \\
1974\end{array}$ \\
\hline $\begin{array}{l}\text { une chaine en or } \\
\text { très fin du poids } \\
\text { de } 225 \text { grammes, } \\
\text { composé de six } \\
\text { grands anneaux } \\
\text { ayant chacun } \\
\text { quatre tours (cette } \\
\text { chaine est très bien } \\
\text { conservée) }\end{array}$ & $\begin{array}{l}\mathrm{n}^{\circ} 1441: \text { chaîne en } \\
\text { or très fin, du poids } \\
\text { de } 225 \text { grammes } \\
\text { composée de six } \\
\text { grands anneaux } \\
\text { ayant chacun } \\
\text { quatre tours. Poids } \\
227 \text { grammes, } \\
9 \text { décigrammes }\end{array}$ & chaîne en or & une chaîne en or & $\begin{array}{l}\text { ornement composé } \\
\text { de bracelets } \\
\text { entrelacés, en or, } \\
\text { Musée de Cluny, } \\
n^{\circ} 1798\end{array}$ & $\begin{array}{l}n^{\circ} 30487 \text { : chaîne } \\
\text { en or fin, du poids } \\
\text { de } 225 \text { grammes, } \\
\text { composée de six } \\
\text { grands anneaux à } \\
\text { quatre tours. } \\
\text { Cette [indication ?] } \\
\text { du catalogue est } \\
\text { fausse ; le poids } \\
\text { n'est que de } \\
19 \text { grammes et il n'y } \\
\text { a qu'un anneau de } \\
4 \text { tours [illisible] }\end{array}$ & $\begin{array}{l}\text { chaîne en or, } \\
\text { Musée de Cluny } \\
N^{\circ} 1441 \text {, } \\
\text { M.A.N. galvano } \\
N^{\circ} 30.487\end{array}$ \\
\hline $\begin{array}{l}\text { une chaine en } \\
\text { argent } \\
\text { fortement oxydée, } \\
\text { et composée d'un } \\
\text { grand anneau et } \\
\text { de deux autres } \\
\text { beaucoup moins } \\
\text { grands }\end{array}$ & $\begin{array}{l}\mathrm{n}^{\circ} 1442 \text { : chaîne en } \\
\text { argent fortement } \\
\text { oxidée composée } \\
\text { d'un grand anneau } \\
\text { et de } 2 \text { autres } \\
\text { plus petits. Poids } \\
130 \text { grammes, } \\
5 \text { décig. brut. }\end{array}$ & & $\begin{array}{l}\text { une chaîne en } \\
\text { argent très oxydée } \\
\text { et fragile comme du } \\
\text { verre. }\end{array}$ & $\begin{array}{l}\text { ornement composé } \\
\text { de bracelets } \\
\text { entrelacés, } \\
\text { en argent. }\end{array}$ & $\begin{array}{l}\mathrm{n}^{\circ} 30488 \text { : chaîne } \\
\text { en argent composé } \\
\text { d'un grand anneau } \\
\text { à } 4 \text { tours. Le } \\
\text { Catal[ogue] indique } \\
\text { fragment d'un } \\
\text { second anneau plus } \\
\text { petit. }\end{array}$ & $\begin{array}{l}\text { parure en argent, } \\
\text { M.A.N. galvano } \\
\mathrm{N}^{\circ} 30.488 \text {. }\end{array}$ \\
\hline $\begin{array}{l}\text { un casse-tête qui } \\
\text { n'est autre chose } \\
\text { qu'un gros caillou } \\
\text { ayant la forme d'une } \\
\text { fève }\end{array}$ & $\begin{array}{l}\mathrm{n}^{\circ} 1443 \text { : un casse- } \\
\text { tête, sous la forme } \\
\text { d'un gros caillou. }\end{array}$ & & & & $\begin{array}{l}\mathrm{n}^{\circ} 30489 \text { : caillou } \\
\text { en silex } \\
\text { (travail humain ??). }\end{array}$ & $\begin{array}{l}\text { «casse-tête ", } \\
\text { M.A.N., n } 30.489 \text {. }\end{array}$ \\
\hline $\begin{array}{l}\text { trois instruments } \\
\text { tranchants } \\
\text { oxydés que bien } \\
\text { des personnes } \\
\text { considèrent comme } \\
\text { des glaives et } \\
\text { d'autres comme des } \\
\text { lances }\end{array}$ & $\begin{array}{l}\mathrm{n}^{\circ} 1444: \text { trois } \\
\text { glaives ou } \\
\text { poignards oxidés. }\end{array}$ & objets en cuivre & $\begin{array}{l}\text { cinq poignards } \\
\text { ou lance en bronze. }\end{array}$ & $\begin{array}{l}\text { [trois] glaives, } \\
\text { Musée de Cluny, } \\
\text { Paris. }\end{array}$ & $\begin{array}{l}\mathrm{n}^{\circ} 30490 \text { : } \\
\text { trois glaives ou } \\
\text { poignards oxydés } \\
\text { portant les traces } \\
\text { d'une couche } \\
\text { d'argent. }\end{array}$ & $\begin{array}{l}\text { lame d'épée, } \\
\text { M.A.N., n } 30.490 \text {. } \\
\text { lame d'épée, } \\
\text { M.A.N., n } 30.490 \text {. } \\
\text { lame d'épée, } \\
\text { M.A.N., n } 30.490 \text {. }\end{array}$ \\
\hline une pique oxydée & $\begin{array}{l}\mathrm{n}^{\circ} 1445 \text { : une pique } \\
\text { oxidée. }\end{array}$ & & & & $\begin{array}{l}\mathrm{n}^{\circ} 30491 \text { : talon } \\
\text { d'arme en bronze } \\
\text { bien patiné. Le } \\
\text { Catal[ogue] dit } \\
\text { " pique » ( ?!). }\end{array}$ & $\begin{array}{l}\text { talon de lance, } \\
\text { M.A.N., n } 30.491\end{array}$ \\
\hline $\begin{array}{l}\text { un petit poignard } \\
\text { oxydé }\end{array}$ & $\begin{array}{l}\mathrm{n}^{\circ} 1446 \text { : un petit } \\
\text { poignard oxidé. } \\
\text { Divers morceaux de } \\
\text { métal. }\end{array}$ & objet en cuivre & & $\begin{array}{l}\text { poignard, } \\
\text { Musée de Cluny, } \\
\text { Paris. }\end{array}$ & $\begin{array}{l}\mathrm{n}^{\circ} 30492 \text { : petit } \\
\text { poignard triangulaire } \\
\text { en bronze. }\end{array}$ & $\begin{array}{l}\text { lame de poignard, } \\
\text { M.A.N., n } 30.492 \text {. }\end{array}$ \\
\hline
\end{tabular}

des Antiquités nationales récemment créé. Cependant tous les objets n'iront pas à Saint-Germain-en-Laye : « parmi les objets des époques préhistoriques il existe des bijoux d'or d'une grande valeur qui, étant aujourd'hui exposés à côté d'œuvres de l'orfèvrerie des Mérovingiens des Carolingiens et des Goths, forment l'introduction d'une série aussi précieuse par la matière que par le travail. On devrait les garder à Cluny d'autant que le musée de Saint-Germain en possède des fac-similés » (Minute de lettre du 27 décembre 1886 d'Alfred Darcel au ministre de l'Instruction publique et des Beaux-arts, archives du musée national du Moyen-Âge de Cluny in Henry, 2008). De ce fait, la chaîne en or de Lothéa est toujours exposée à Cluny, tandis que le reste du mobilier, rayé de l'inventaire, a rejoint en 1887 le musée d'Archéologie nationale, sous les numéros 30487 à 30 494. Par la suite, les objets de Lothéa sont exposés dans la salle III du musée de Saint-Germain-en-Laye (Reinach, 1898, p. 70). En 1994, le mobilier de la sépulture de Lothéa est encore exposé dans la salle III du musée de Saint-Germain-en-Laye
(Mohen et al., 1994, p. 87). Il a aujourd'hui disparu de l'actuelle muséographie. Le catalogue de 1898 semble être le dernier témoignage des pointes de flèches (Reinach, 1898), qui n'ont pu être retrouvées au musée d'Archéologie nationale. Pour ce qui est des chaînes en or et en argent, il semble qu'il y ait eu quelques confusions entre les inventaires, les objets et leurs fac-similés. L'ensemble du mobilier de Lothéa a été reporté au catalogue du musée d'Archéologie nationale, sans prendre en compte le fait que la chaîne en or soit restée au musée de Cluny (tabl. I). Une note portée à l'inventaire précise que « les $n^{\circ} 30487$ et 30488 [la chaîne en or et celle en argent] ont été vainement cherchés en octobre 1911 ॥. La chaîne en argent semble s'être rapidement détériorée, elle est décrite comme " fortement oxydée » dans l'inventaire du musée de Cluny. Elle est manifestement disloquée quand elle arrive à SaintGermain-en-Laye ; en effet, il est précisé à l'inventaire qu'il manque un anneau à la chaîne en argent, tandis que probablement le second anneau est compris comme étant ce qui subsiste 
Tabl. I (suite) - Comparaisons entre les différents inventaires du mobilier du tumulus de Lothéa (Quimperlé, Finistère).

Comparisons between the different inventories of grave goods from the Lothéa barrow (Quimperlé, Finistère).

\begin{tabular}{|c|c|c|c|c|c|c|}
\hline Boutarel, 1843 & $\begin{array}{l}\text { Inventaire du Musée } \\
\text { de Cluny, février } \\
1844\end{array}$ & Taylor et al., 1846 & Le Men, 1868 & $\begin{array}{l}\text { Micault, } 1881 \text { et } \\
\text { Société d'Émulation } \\
\text { des Cotes-du-Nord, } \\
1884\end{array}$ & $\begin{array}{l}\text { Inventaire du Musée } \\
\text { d'Archéologie } \\
\text { Nationale, } 1887\end{array}$ & $\begin{array}{l}\text { Briard et Mohen, } \\
1974\end{array}$ \\
\hline $\begin{array}{l}\text { deux morceaux } \\
\text { de métal } \\
\text { oxydé, formant } \\
\text { probablement les } \\
\text { débris de quelques } \\
\text { armes }\end{array}$ & $\mathrm{n}^{\circ} 1446 ?$ & & & & & \\
\hline $\begin{array}{l}\text { une jolie pierre } \\
\text { rouge qui a la forme } \\
\text { d'un quadrilatère } \\
\text { rectangle. Elle a un } \\
\text { trou au sommet de } \\
\text { chaque angle. Elle } \\
\text { est probablement } \\
\text { une ancienne fronde }\end{array}$ & $\begin{array}{l}\mathrm{n}^{\circ} 1447: \text { pierre } \\
\text { rouge ayant } \\
\text { la forme d'un } \\
\text { quadrilatère } \\
\text { rectangle percée } \\
\text { d'un trou au sommet } \\
\text { de chaque angle. }\end{array}$ & porphyre rouge & $\begin{array}{l}\text { une pierre rouge } \\
\text { ayant la forme } \\
\text { d'un quadrilatère } \\
\text { rectangle, percée } \\
\text { d'un trou au sommet } \\
\text { de chaque angle. }\end{array}$ & & $\begin{array}{l}\mathrm{n}^{\circ} 30492 \\
\text { bis: plaquette } \\
\text { rectangulaire en } \\
\text { pierre rouge percée } \\
\text { de trous aux quatre } \\
\text { angles. }\end{array}$ & $\begin{array}{l}\text { brassard d'archer, } \\
\text { M.A.N., n } 30.492 \\
\text { bis. }\end{array}$ \\
\hline $\begin{array}{l}\text { une amulette en } \\
\text { pierre verte taillée, } \\
\text { ayant la forme } \\
\text { d'un trapèze dont } \\
\text { les deux bases } \\
\text { parallèles sont } \\
\text { arrondies. Cette } \\
\text { amulette a un trou } \\
\text { très régulièrement } \\
\text { fait }\end{array}$ & $\begin{array}{l}\mathrm{n}^{\circ} 1448: \text { sorte } \\
\text { d'amulette en pierre } \\
\text { verte taillée ayant la } \\
\text { forme d'un trapèze } \\
\text { dont les deux bases } \\
\text { parallèles sont } \\
\text { arrondies. Cette } \\
\text { pierre est percée } \\
\text { d'un trou. }\end{array}$ & jaspe vert & $\begin{array}{l}\text { une pierre verte } \\
\text { percée d'un trou. }\end{array}$ & $\begin{array}{l}\text { une pendeloque en } \\
\text { jadéite }\end{array}$ & $\begin{array}{l}\mathrm{n}^{\circ} 30493 \text { : amulette } \\
\text { en pierre verte } \\
\text { jaspoïde, percée } \\
\text { d'un trou au } \\
\text { sommet. }\end{array}$ & $\begin{array}{l}\text { pendentif, } \\
\text { M.A.N., n } 30.493\end{array}$ \\
\hline $\begin{array}{l}7 \text { flèches en silex, } \\
\text { et dentelées, qui } \\
\text { annoncent que } \\
\text { le tombeau dont } \\
\text { il s'agit, date } \\
\text { d'une époque très } \\
\text { reculée, et qu'il est } \\
\text { même antérieur à } \\
\text { la fondation de la } \\
\text { monarchie }\end{array}$ & $\begin{array}{l}\mathrm{n}^{\circ} 1449 \text { : flèches } \\
\text { en silex dentelées } \\
\text { d'une forme très } \\
\text { antique, antérieure } \\
\text { à la fondation de la } \\
\text { monarchie. }\end{array}$ & $\begin{array}{l}\text { armatures de } \\
\text { flèches en silex (2 } \\
\text { sont représentées) }\end{array}$ & $\begin{array}{l}\text { des pointes de } \\
\text { flèches en silex } \\
\text { dentelées, au } \\
\text { nombre de dix à } \\
\text { douze suivant les } \\
\text { uns, d'une vingtaine } \\
\text { suivant les autres. }\end{array}$ & $\begin{array}{l}\text { un certain nombre } \\
\text { de pointes de } \\
\text { flèches en silex, au } \\
\text { nombre de six ou } \\
\text { sept. }\end{array}$ & $\begin{array}{l}\mathrm{n}^{\circ} 30494: \text { sept } \\
\text { flèches barbelées à } \\
\text { pédoncule. }\end{array}$ & \\
\hline \multirow{5}{*}{$\begin{array}{l}\text { une petite baguette } \\
\text { en cuivre }\end{array}$} & $\mathrm{n}^{\circ} 1446 ?$ & objet en cuivre & & & & \\
\hline & & objet en cuivre & $\begin{array}{l}\text { une hache en } \\
\text { bronze (disparue) }\end{array}$ & $\begin{array}{l}\text { une hache en } \\
\text { bronze à ailerons, } \\
\text { faisant partie de la } \\
\text { même trouvaille, } \\
\text { aujourd'hui disparue }\end{array}$ & & \\
\hline & & & & $\begin{array}{l}\text { bracelet en bronze, } \\
\text { coll. Fornier, } \\
\text { Rennes. }\end{array}$ & & $\begin{array}{l}\text { bracelet, Musée de } \\
\text { Bretagne à Rennes }\end{array}$ \\
\hline & & & & $\begin{array}{l}\text { chaîne en bronze, } \\
\text { coll. Fornier, } \\
\text { Rennes }\end{array}$ & & $\begin{array}{l}\text { parure en bronze, } \\
\text { connue par } \\
\text { les Trésors de } \\
\text { l'Armorique }\end{array}$ \\
\hline & & & & & & $\begin{array}{l}\text { pointe de flèche } \\
\text { à barbelures et } \\
\text { pédoncule, Musée } \\
\text { Préhistorique } \\
\text { Finistérien }\end{array}$ \\
\hline
\end{tabular}

de la chaîne en or - cette dernière se trouvant à Cluny. En 1917, il est précisé que la chaîne en argent exposée est un fac-similé (Reinach, 1917, p. 39). Deux clichés témoignent des fac-similés des chaînes en or et en argent (Giot et al., 1979, p. 55 ; Eluère, 1982, p. 133), tandis que Jacques Briard et Jean-Pierre Mohen (1974) précisent qu'il s'agit de galvanoplasties ; celles-ci n’ont pu être retrouvées. Enfin il reste un « casse-tête » ou gros galet inscrit aux inventaires et vu pour la dernière fois en 1974 (Briard, Mohen, 1974).

En fin de compte, si certains objets ont disparu, ceux-ci n'ont jamais quitté la région parisienne. Ils sont aisément identifiables grâce aux multiples descriptions, dessins ou photographies et constituent les vraies reliques, les véritables objets provenant de la tombe de Lothéa. Nous nous tiendrons à la description d'Arthur Boutarel (tabl. I), à laquelle il est possible d'ajouter la hache à légers rebords signalée par René-François Le Men (1868), dont l'histoire rocambolesque parait vraisemblable (cf. supra) ; d'autant qu'un autre objet, le petit poignard oxydé, fut également subtilisé avant de se retrouver dans les collections du musée de Cluny (Lettre du 19 août 1843). 

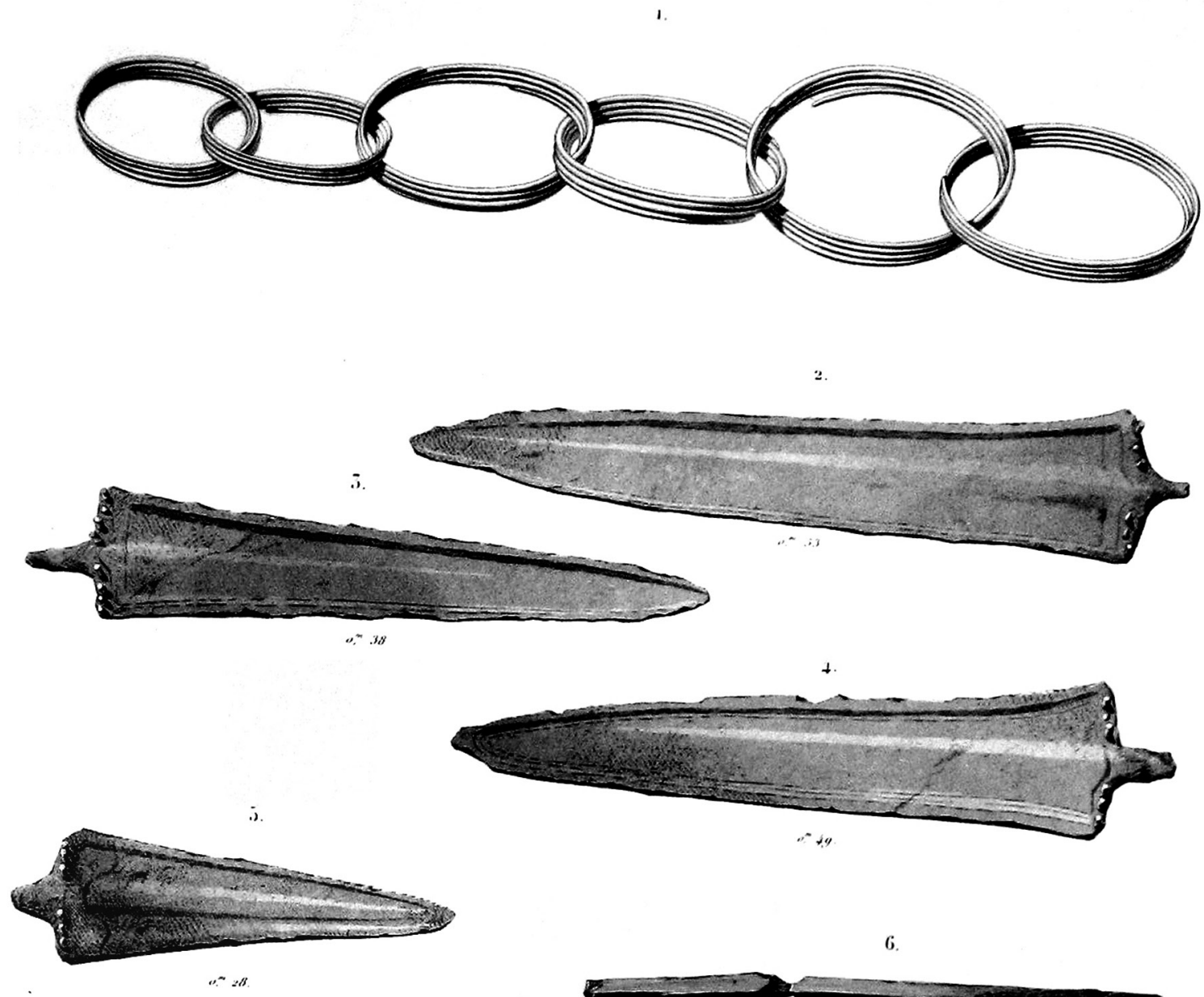

6.
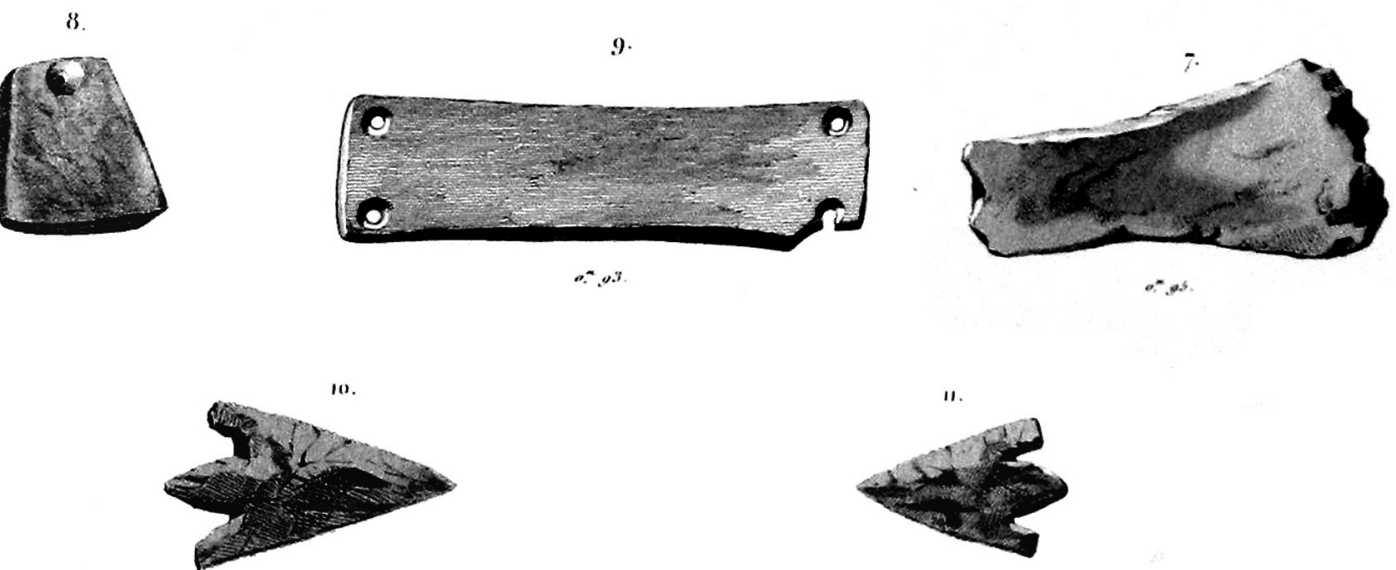

FOLIILIF EATE DANS I.E TIMULIS DE CARNOLET

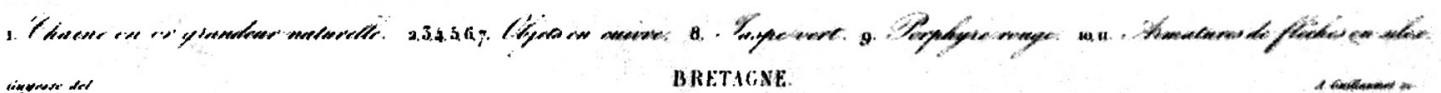

Fig. 3 - Fouille faite dans le tumulus de Carnoët (d'après Taylor et al., 1846). Barrow excavation at Carnoët (after Taylor et al., 1846). 

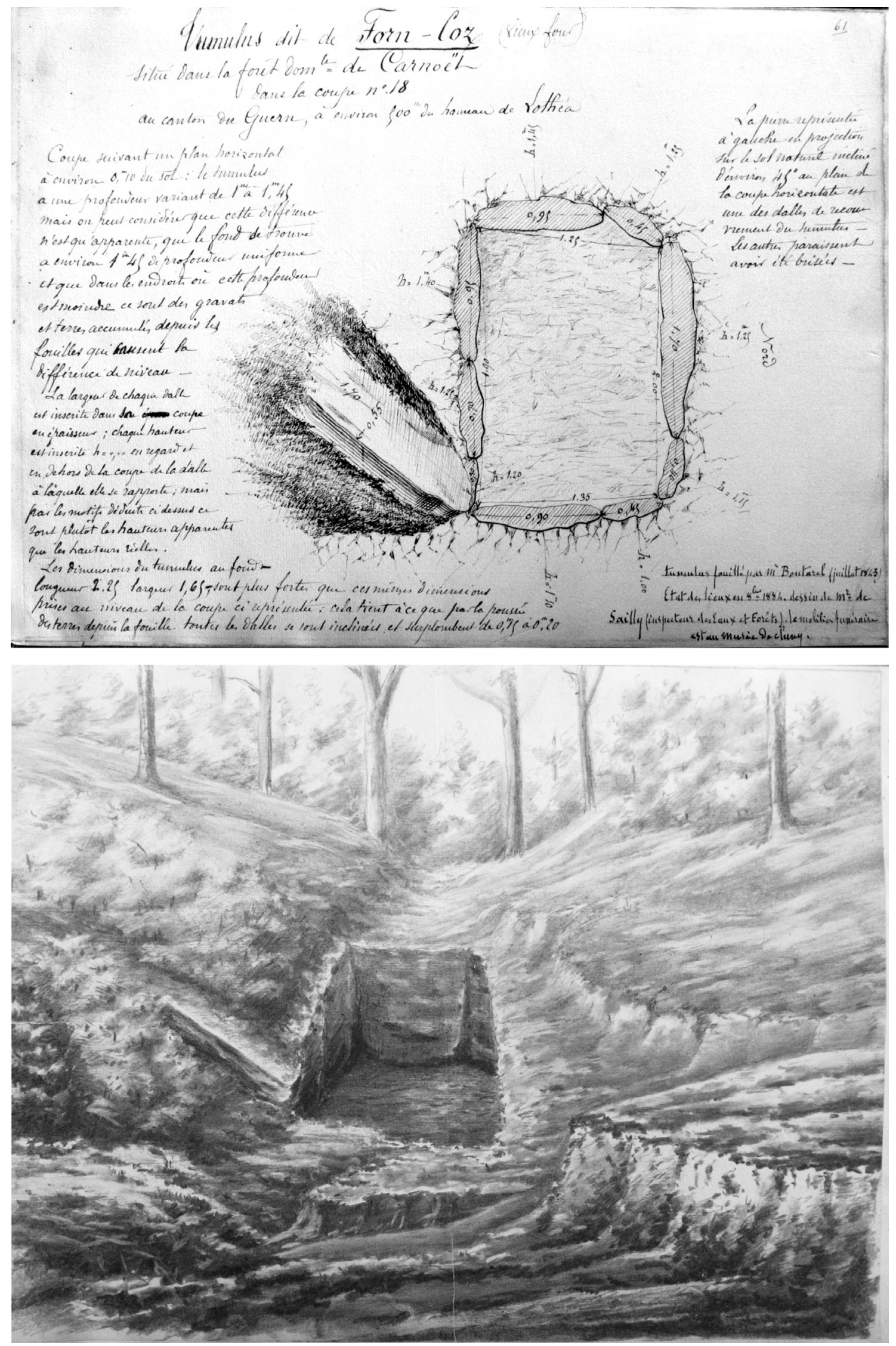

Fig. 4 - Le tumulus de Lothéa en 1884 par M. de Sailly, inspecteur des Eaux et forêts (d'après Chatellier, archives).

The Lothéa barrow in 1884 by M. de Sailly, forest inspector (after Chatellier, archives). 


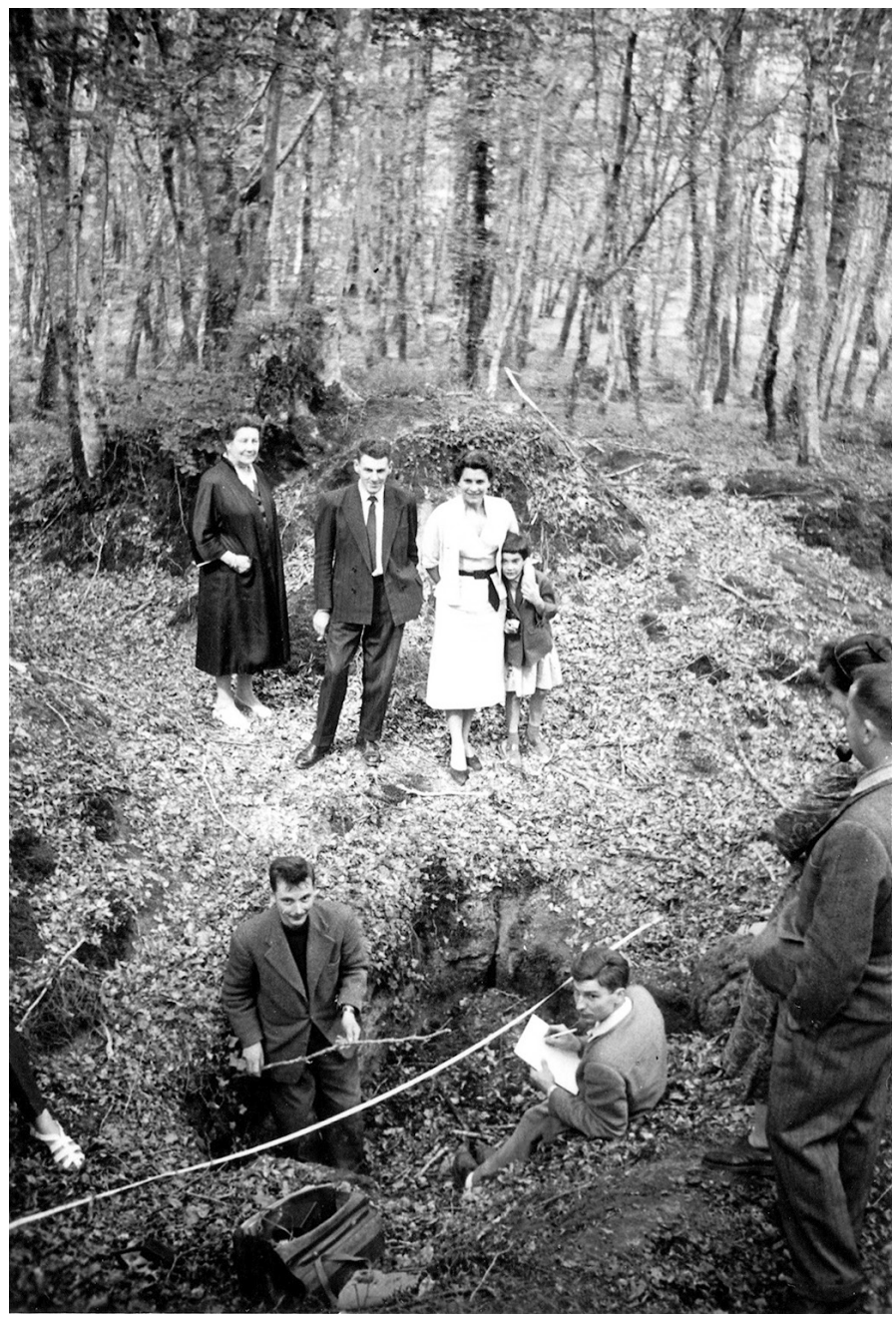

Fig. 5 - Jacques Briard (en bas à gauche) et Jean L'Helgouach (en bas à droite) effectuant le relevé du coffre de Lothéa, le 14 juillet 1957 (archives du laboratoire Archéosciences - UMR6566/CreAAH).

Jacques Briard (down left) and Jean L'Helgouach (down right) doing the plan of the Lothéa cist, the July 14th 1957 (laboratoire Archéosciences - UMR6566/CreAAH archives).

Il reste la chaîne et la spirale en bronze de la collection La Gillardaie et la flèche du musée de Préhistoire finistérienne qui par leur nature et leur histoire sont en contradiction avec les vraies reliques de Lothéa. Les deux parures en bronze sont de factures et de matières différentes des deux chaînes en métaux précieux (or et argent). La spirale en bronze est faite suivant les dessins d'un fin ruban (Société d'Emulation des Côtes-du-Nord, 1884) ou d'un fil (Briard, Mohen, 1974), tandis que la chaîne en bronze allie spirales et anneaux massifs (fig. 6, nos 1 et 2). Elles ne sont signalées qu'une quarantaine d'années après leur découverte supposée et sont passées, comme la hache à rebords, dans les collections de La Gillardaie, de Fornier (Micault, 1881) et, enfin, celle du Musée de Bretagne à Rennes où seule la spirale est conservée (Briard et al., 1977b). Ces observations faites, il semble plutôt s'agir d'un mélange dans les collections que d'objets provenant de la tombe de Lothéa. La flèche du musée de Penmarc'h est du type armoricain (fig. 6, $n^{\circ} 3$ ), caractéristique de l'Âge du Bronze ancien (Briard, Mohen, 1974, p. 49), mais ne correspond pas aux dessins faits au XIX ${ }^{\mathrm{e}}$ siècle (fig. 3). Elle
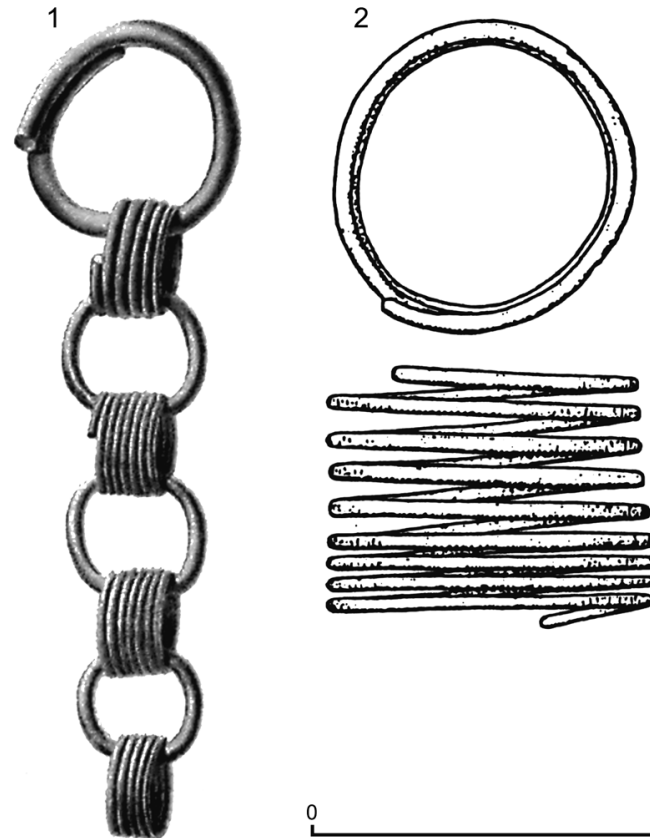

3
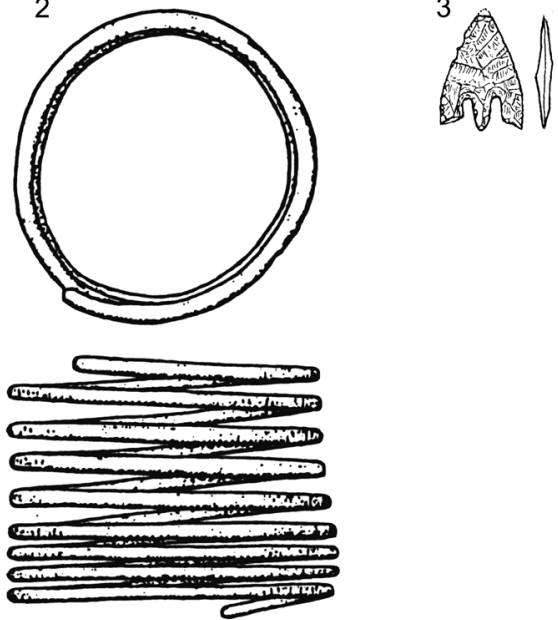

Fig. 6 - Les «fausses reliques » de Lothéa. $N^{\circ} 1$ : chaîne en bronze de la collection Fornier; $n^{\circ} 2$ : bracelet spiralé en bronze du musée de Rennes ; $n^{\circ} 3$ : flèche du musée de Penmarc'h ( $n^{\circ} 1$ d'après Société d'Émulation des Côtes-du-Nord, 1884, $n^{\text {os }} 2$ et 3 d'après Briard et Mohen, 1974).

The Lothéa "fake relics". $N^{\circ} 1$ : bronze chain from Fornier collection ; $n^{\circ} 2$ : bronze spiraled bracelet from Rennes museum; $n^{\circ} 3$ : arrowhead from Penmarc'h museum ( $n^{\circ} 1$ after Société d'Émulation des Côtes-duNord, 1884, $n^{o s} 2$ and 3 after Briard and Mohen, 1974).

est mentionnée comme étant une " pointe de flèche en silex, que l'on suppose venir du tumulus de Carnoët (Don de M. le Comte A. de Blois) » (Serret, 1901, p. 19). Rien ne permet de confirmer l'origine de cette flèche du musée de Quimper, puis de Penmarc'h. Il ne semble pas y avoir eu plus de sept flèches découvertes dans la tombe de Carnoët - cela étant confirmé par deux témoignages - et ces sept flèches se trouvaient dans les collections du musée d'Archéologie nationale (tabl. I).

\section{LES DEUX TUMULUS DE LA FORÊT DE CARNOËT}

La forêt de Carnoët remonte au moins au XII siècle et sa configuration a peu évolué depuis le XVIII ${ }^{\mathrm{e}}$ siècle, comme l'attestent les cartes des Cassini. Outre les deux tumulus de l'Âge du Bronze, cette forêt renferme des vestiges remontant aux époques néolithiques (dolmen de Toulfoën), gauloises (stèles) et romaines (thermes) ainsi que divers talus, édifiés, en toute logique, avant le XII ${ }^{\mathrm{e}}$ siècle (Le Roux, 1981 ; Paulet, 2005 ; Galliou, 2010). L'absence d'agriculture pendant neuf siècles et surtout de labours profonds a permis une conservation remarquable des tumulus ; tandis que la grande majorité d'entre eux a aujourd'hui disparu ou n'est réduite qu'à l'état de buttes très arasées. 


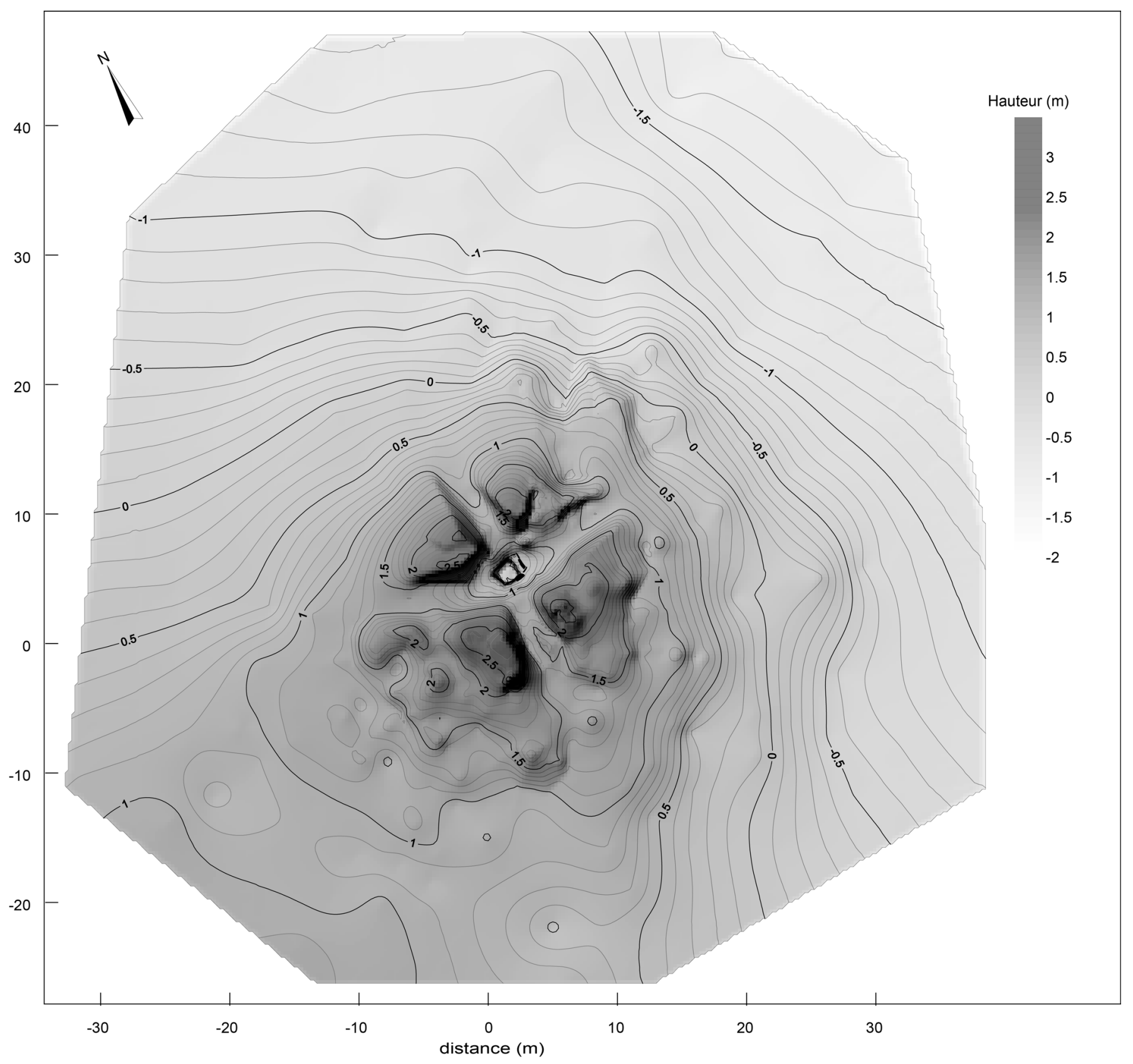

Fig. 7 - Relevé topographique du tumulus de Lothéa, Quimperlé, Finistère (relevé : P. Stephan, CNRS, T. Kervern).

Topographic plan of the Lothéa barrow, Quimperlé, Finistère (plan: P. Stephan, CNRS, T. Kervern).

\section{LE TUMULUS DE LOTHÉA}

Coordonnées Lambert II étendu : $\mathrm{X}: 160480 ; \mathrm{Y}: 2332515 ; \mathrm{Z}: 58$

Afin de mieux appréhender les structures du tumulus, un relevé topographique du site a été effectué à l'aide d'un tachéomètre laser de type Leica DTM 300 (fig. 7) avec une précision de $\pm 2 \mathrm{~cm}$ dans les trois dimensions. Malgré son intérêt, l'utilisation du GPS différentiel a dû être écartée dans notre cas, la présence d'une canopée même modeste empêchant toute mesure de ce type. À partir des données topographiques, un modèle numérique de terrain a été réalisé sous le logiciel
Surfer 8.0 à une résolution de $25 \mathrm{~cm}$ afin de reconstituer le plus finement possible la morphologie générale du secteur.

Dans son état actuel, le tumulus mesure $52 \mathrm{~m}$ dans son diamètre NO-SE et $45 \mathrm{~m}$ dans son diamètre NE-SO pour une hauteur maximum de $2,85 \mathrm{~m}$ (fig. 8). Il porte encore les stigmates de la fouille de 1843 qui correspondent à deux grandes tranchées de 3 à $4 \mathrm{~m}$ de largeur, se croisant et s'élargissant vers le centre (fig. 8 et 9). Le tumulus a largement été agrandi par les déblais de fouilles rejetés à la sortie des tranchées. Le volume actuel du tumulus, en incluant les déblais de fouilles, est de 1580 $\pm 32 \mathrm{~m}^{3}$. Ce résultat n'est évidemment qu'une approximation de la réalité. Il a été obtenu par soustraction numérique de la topographie actuelle (avec tumulus) à la topographie originelle, c'est- 
à-dire celle précédant l'édification du tumulus. Cette ancienne topographie a été reconstituée en considérant comme régulière la pente du versant. La marge d'erreur associée au calcul du volume a été obtenue en multipliant la superficie du tumulus $\left(1600 \mathrm{~m}^{2}\right)$ par l'incertitude de la mesure topographique $(2 \mathrm{~cm})$. Le calcul du volume et la comparaison avec d'autres tumulus fouillés anciennement permet de restituer un tumulus d'environ $26 \mathrm{~m}$ de diamètre et $4 \mathrm{~m}$ de hauteur, soit exactement l'estimation de René-François Le Men (1868, p. 365) et sensiblement inférieur aux $40 \mathrm{~m}$ de diamètre et $6 \mathrm{~m}$ de hauteur proposés par Arthur Boutarel (Lettre du 19 août 1843).

Le caveau est constitué de neuf pierres de chant en orthogneiss à gros grain (détermination L. Chauris) (fig. 10). Cette pierre est disponible localement, formant l'essentiel du socle rocheux de la forêt de Carnoët. Elle est issue de phénomènes d'écrasement et de recristallisation du granite de Moëlan (Cogné et al., 1973). Le coffre est orienté est-ouest et mesure 2,5 $\mathrm{m}$ de long pour $2 \mathrm{~m}$ de large. Dans son état actuel, l'espace interne fait 2,15 $\mathrm{m}$ de long pour 1,45 $\mathrm{m}$ de large. Un plan réalisé en 1884 par de Sailly, inspecteur des Eaux et Forêts, montre qu'à la base l'espace devait être un peu plus grand avec 2,25 m de long pour 1,65 $\mathrm{m}$ de large (fig. 4). Les dalles de chant sont observables sur une hauteur de $0,6 \mathrm{~m}$, mais celles-ci sont recouvertes par une épaisse couche d'humus (fig. 9). Grâce au plan levé par de Sailly, nous savons que ces dalles mesuraient entre 1 et 1,45 $\mathrm{m}$ de hauteur (fig. 4). Trois modules de pierres ont été employés. Une première dalle, disposée au nord, mesure près d'1,8 $\mathrm{m}$ de large, quatre autres dalles, formant les parois ouest, sud et est, font entre $0,77 \mathrm{~m}$ et $0,87 \mathrm{~m}$ de large. Enfin quatre dalles mesurant entre $0,44 \mathrm{~m}$ et $0,52 \mathrm{~m}$ forment trois des angles recouvrant les autres pierres (angles nord-ouest et sud-est) ou se chevauchant (angle nord-est). Le relevé topographique montre que les dalles du coffre sont théoriquement plantées sur environ un tiers de leur hauteur dans le sol (fig. 8). Les témoins interrogés par René-François Le Men rapportent la possible existence d'un pavage situé au milieu du coffre composé d'une rangée de cinq à six pierres plates, mesurant chacune environ 0,3 m de grandeur (Le Men, 1868, p. 366). Cependant ce pavage n'apparaît ni sur le plan de J. W. Lukis dressé un an après la fouille (fig. 2), ni sur celui de De Sailly (fig. 4). Un morceau de la dalle de couverture en orthogneiss est conservé, aujourd'hui replacé, il était auparavant renversé près de la tombe (fig. 4). Il mesure 1,77 $\mathrm{m}$ de longueur pour $0,67 \mathrm{~m}$ de largeur et $0,23 \mathrm{~m}$ d'épaisseur. Ces dimensions correspondent parfaitement à une extrémité d'une dalle qui mesurait 2,2 $\mathrm{m}$ de longueur, 1,8 $\mathrm{m}$ de largeur et 0,45 $\mathrm{m}$ d'épaisseur selon Arthur Boutarel (Lettre du 19 août 1843). Au regard du plan de la tombe, cette dalle de couverture devait être suffisante pour coiffer les dalles de chant sans toutefois les déborder (fig. 10).

Un cairn entourait et recouvrait le coffre, mais il reste difficile à appréhender. Lors de la fouille, Arthur Boutarel le décrit comme : « une grande quantité de moëllons placés pêle-mêle les uns sur les autres sans former la moindre maçonnerie » (Lettre du 19 août 1843). Il est encore en partie visible dans la coupe nord de la tranchée ouest et il est composé de petits blocs en orthogneiss qui mesurent de 15 à $20 \mathrm{~cm}$. Au niveau de la coupe, le cairn semble redescendre vers le centre. Si l'on admet que le cairn repose sur le sol, comme c'est généralement le cas (Briard, 1984), celui-ci mesurerait environ
$1,7 \mathrm{~m}$ de hauteur ; son diamètre, plus difficilement appréciable, devrait faire un minimum de $8 \mathrm{~m}$ (fig. 8). Cela ferait du cairn de Lothéa l'un des plus massifs connus pour les tumulus en Bretagne. Cependant ces amoncellements de pierre sont souvent assez mal décrits, en raison de fouilles expéditives en puits ou en tranchées. A titre de comparaison, on peut citer le tumulus nord de Kervini (Poullan-sur-Mer, Finistère) avec un cairn de $18 \mathrm{~m}$ de diamètre et $2 \mathrm{~m}$ de hauteur (Briard, 1972) et celui de La Motta (Lannion, Côtes d'Armor) avec un cairn de $14 \mathrm{~m}$ de diamètre et $1 \mathrm{~m}$ de hauteur (Butler, Waterbolk, 1974). Le reste du tertre est constitué d'une couche homogène de terre jaune argilo-limoneuse. Cette dernière est aujourd'hui entremêlée dans sa partie supérieure de terre végétale sur une épaisseur de 40 à $70 \mathrm{~cm}$ (fig. 8).

\section{LE TUMULUS DE KERIVOALEN}

\author{
Coordonnées Lambert II étendu : \\ $\mathrm{X}: 158610 ; \mathrm{Y}: 2331100 ; \mathrm{Z}: 64$.
}

Le tumulus de Kerivoalen apparaît moins étalé et plus petit que celui de Lothéa. Il mesure $19 \mathrm{~m}$ de diamètre et $1,7 \mathrm{~m}$ de hauteur (fig. 9). Il est également entaillé de deux tranchées nord-sud et est-ouest d'1,5 m de largeur environ et qui atteignent 2,1 m de profondeur vers le centre. Ces deux tranchées perpendiculaires signent de toute évidence le passage d'Arthur Boutarel. La fouille de ce tumulus n'a révélé aucune structure interne et a livré très peu de mobilier, mis à part quelques tessons et des charbons provenant des terres du tumulus (Arthur Boutarel, lettre du 18 avril 1845). De tels tumulus sont connus à l'Âge du Bronze. Il peut s'agir de sépultures en pleine terre - le squelette et l'éventuel mobilier en métal pouvant alors être entièrement dissous par l'acidité du sol - mais on ne peut exclure l'existence de tertres à vocation non-funéraire.

\section{DESCRIPTION DU MOBILIER DE LOTHÉA}

\section{LA CHAîNE EN OR (FIG. 11 ET FIG. 12, N 1 )}

Longueur $273 \mathrm{~mm}$; poids $227,9 \mathrm{~g}$;

diamètre et épaisseur des anneaux : $\mathrm{n}^{\circ} 1: 61 \times 2,8 \mathrm{~mm}$; $\mathrm{n}^{\circ} 2: 59 \times 3 \mathrm{~mm} ; \mathrm{n}^{\circ} 3: 50 \times 2,9 \mathrm{~mm} ; \mathrm{n}^{\circ} 4: 49 \times 3 \mathrm{~mm}$; $\mathrm{n}^{\circ} 5: 41 \mathrm{~mm} \times 2,1 \mathrm{~mm} ; \mathrm{n}^{\circ} 6: 41-43 \mathrm{~mm} \times 2,5 \mathrm{~mm}$.

La chaîne en or est très bien conservée. Elle est composée de six anneaux de taille décroissante, de 61 à $41 \mathrm{~mm}$, mais qui s'associent par paire $\left(n^{\circ} 1\right.$ et $2, n^{\circ} 3$ et $4, n^{\circ} 5$ et 6$)$. Le sixième anneau, déformé, adopte une forme légèrement ovalaire. Les tiges d'or ont une épaisseur maximum de 2,5 à $3 \mathrm{~mm}$ s'affinant régulièrement pour se terminer en pointes. Pour le reste, nous n'avons rien à ajouter à l'étude de Christiane Eluère de cette chaîne : «Chacun des six anneaux [...] est fait d'un fil enroulé en quatre spires superposées. Les fils sont d'une exécution parfaite ; à leur face externe, de fines traces de polissage longitudinal sont bien visibles. L'observation à la loupe binoculaire de ces fils indique qu'ils ont été obtenus par le martelage d'une tige de section quadrangulaire, cette forme étant encore per- 


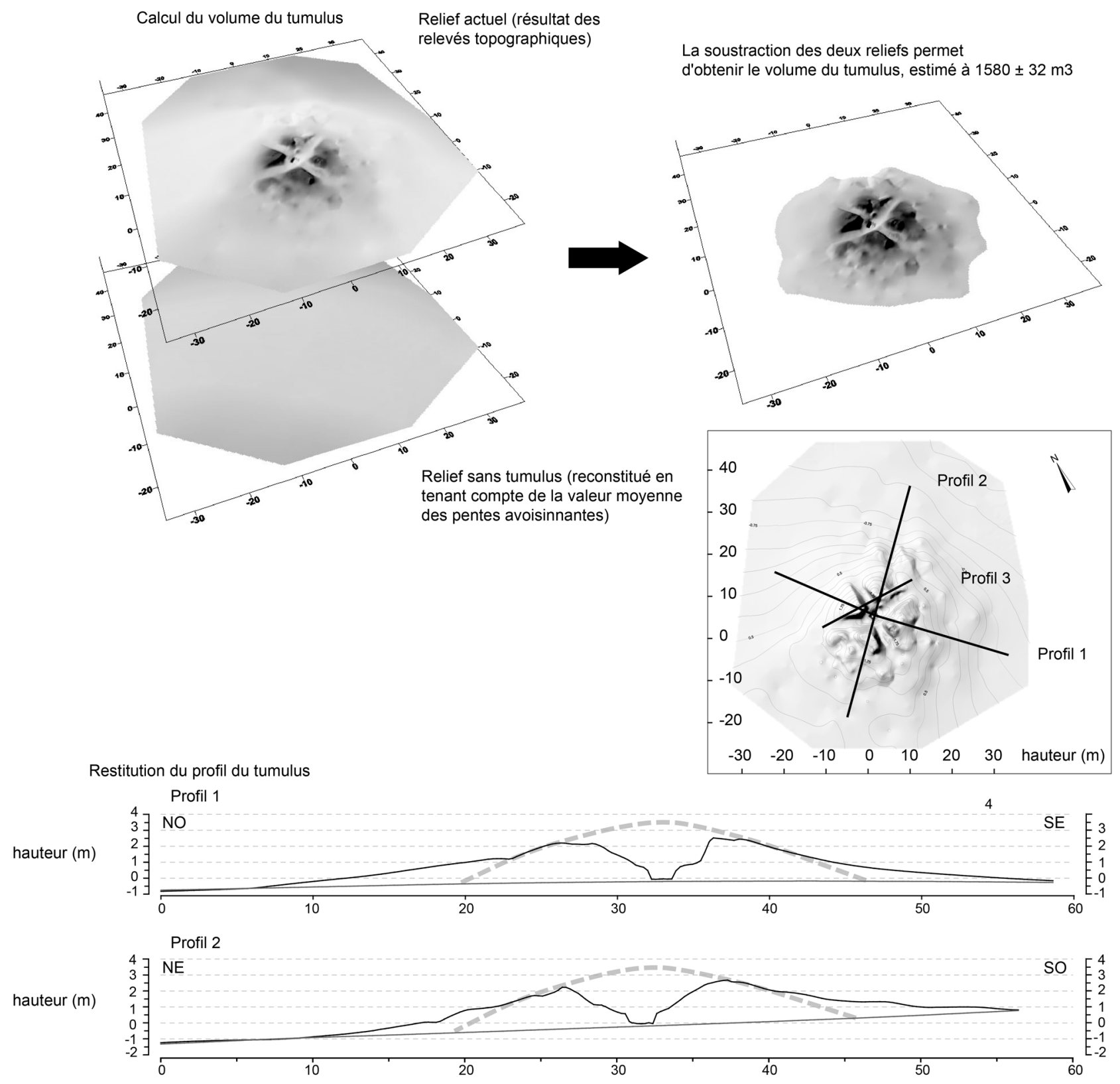

Coupe hypothétique du tumulus

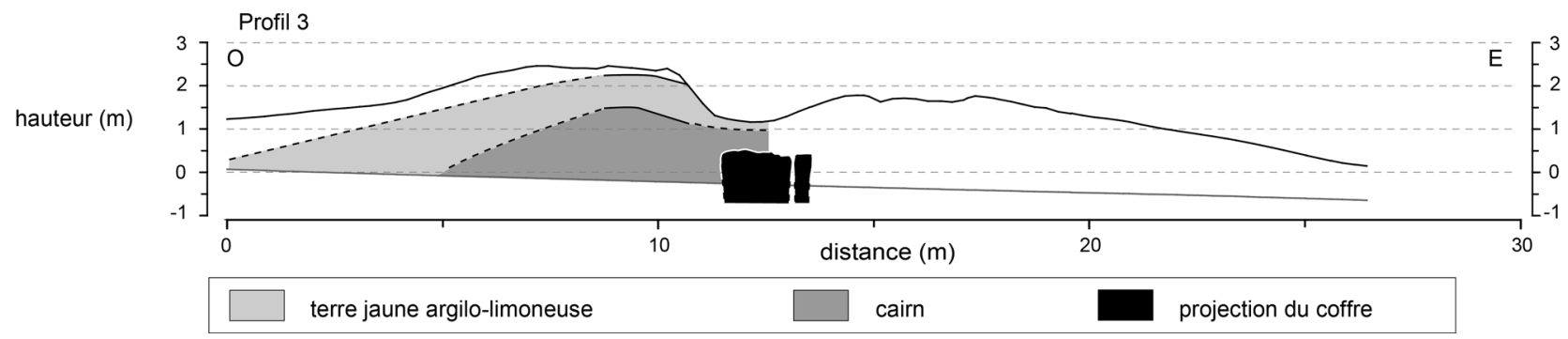

Fig. 8 - Calcul du volume, restitution du profil et coupe hypothétique du tumulus de Lothéa, Quimperlé, Finistère (DAO : P. Stephan, CNRS, C. Nicolas, Université Paris I).

Volumetric calculation, profile restitution and hypothetical section of the Lothéa barrow, Quimperlé, Finistère (CAD: P. Stephan, CNRS, C. Nicolas, Université Paris I). 


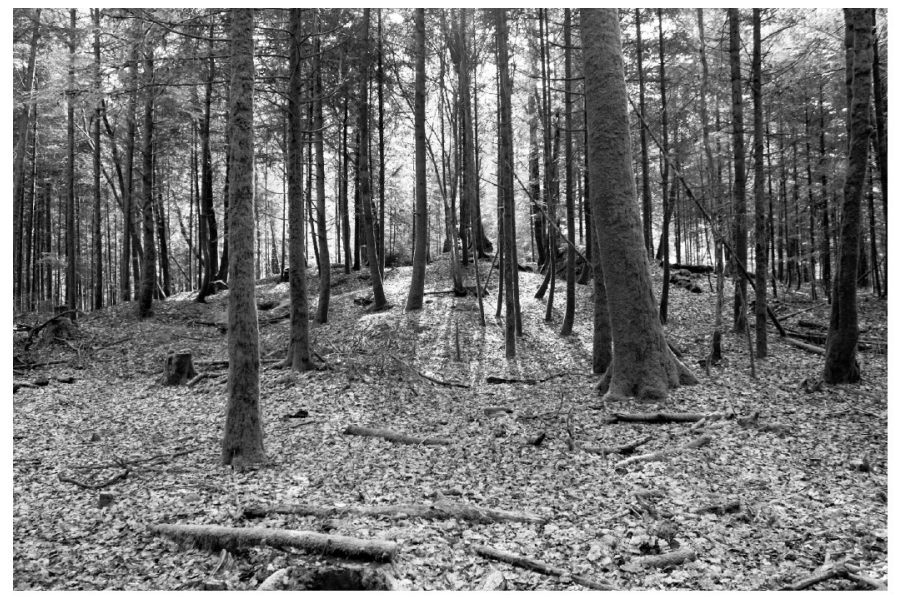

Tumulus de Lothéa, vue nord-est du tumulus

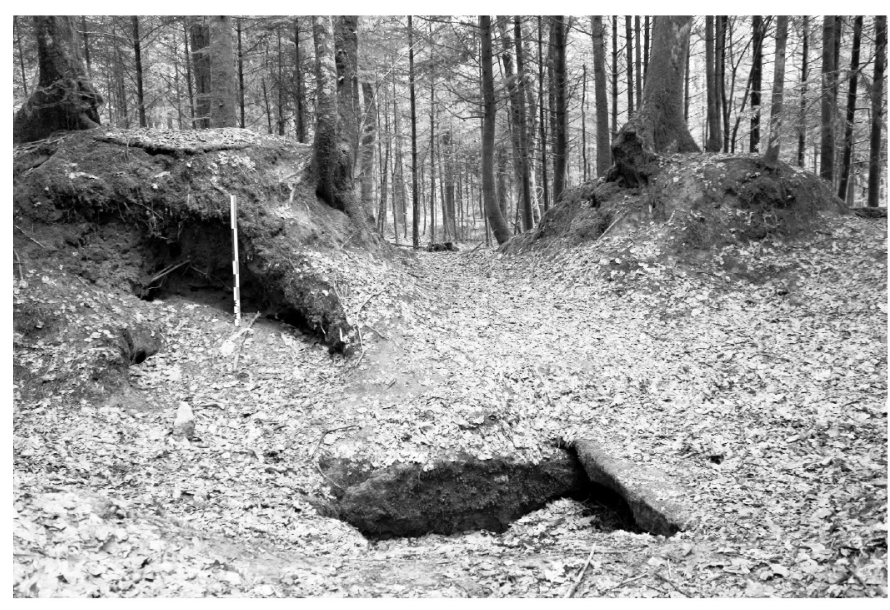

Tumulus de Lothéa, vue du coffre et de la tranchée nord

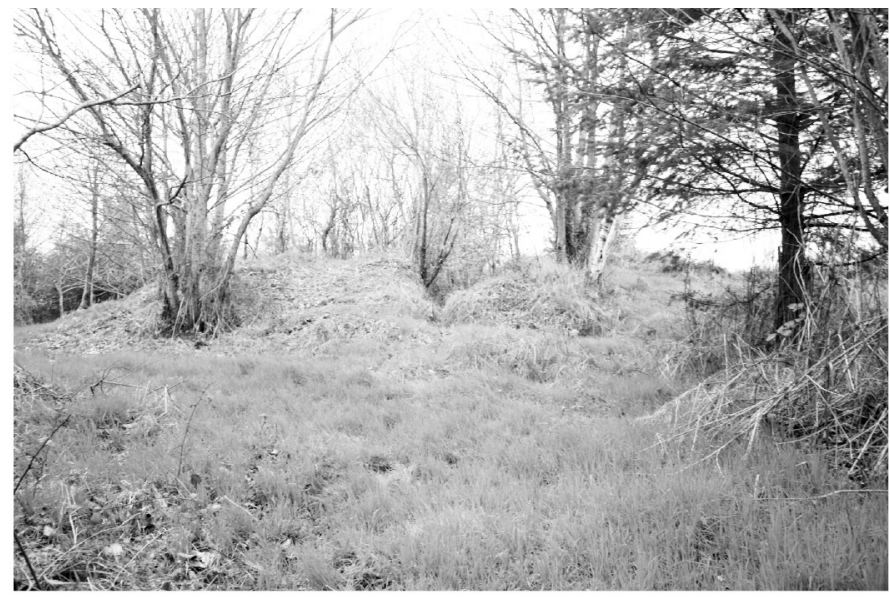

Tumulus de Kerivoalen, vue est

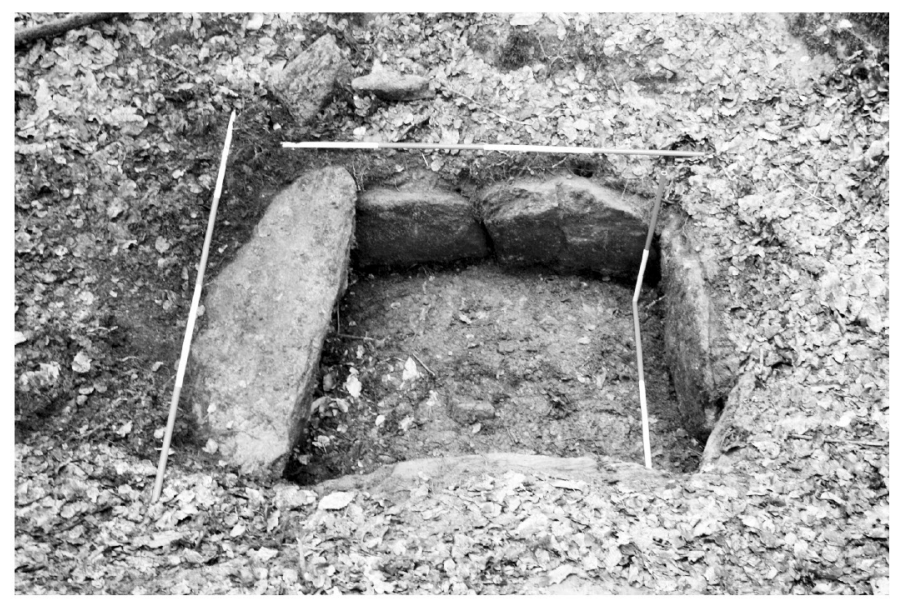

Tumulus de Lothéa, vue du coffre depuis le nord

Fig. 9 - Photographies des deux tumulus de la forêt de Carnoët, Quimperlé, Finistère (clichés : C. Nicolas, Université Paris I).

Photographs of the two barrows of the Carnoët forest, Quimperlé, Finistère (photographs: C. Nicolas, Université Paris I).

ceptible sur les faces supérieure et inférieure de chaque spire, cachées du fait de leur superposition les unes aux autres, sauf si on écarte légèrement les fils. Le polissage soigné des fils n'est donc intervenu qu'après la constitution des enroulements et alors qu'ils étaient maintenus très serrés ; cette opération a été plus qu'une simple finition puisqu'elle a joué un rôle dans l'arrondissement externe des fils. » (Eluère, 1986, p. 97-98). Une partie du fil de l'anneau $\mathrm{n}^{\circ} 5$ présente une facette plane non arrondie bien visible (fig. $13, \mathrm{n}^{\circ} 1$ ).

\section{LA CHAîNe EN ARGENT (FIG. 12, $\mathrm{N}^{\circ}$ 2)}

\section{Poids 130,5 gr.}

La chaine en argent, faite d'anneaux de quatre spires, est très proche de celle en or. Les représentations dont nous disposons divergent sur l'extrémité des fils. En effet le dessin de la Société d'Emulation des Côtes-du-Nord (1884) ainsi que la galvanoplastie (Briard, Mohen, 1974) montrent des extrémités abruptes. En revanche, le dessin d'Adrien de Mortillet (1903) illustre clairement l'extrémité appointée des fils d'argent (fig. $12, n^{\circ} 2$ ). Il montre également les anneaux enchaînés un à un, tandis que la galvanoplastie montre les deux petits anneaux enchaînés au plus grand. Cette divergence s'explique vraisemblablement par le fait qu'un des petits anneaux était détaché quand il est arrivé au musée de Saint-Germain-en-Laye (cf. supra). L'enquête de René-François Le Men peut suggérer que cette chaîne en argent était plus grande à l'origine : « Elle était formée de plusieurs anneaux dont quelques-uns furent détachés par les personnes présentes. On en a conservé longtemps des fragments à Quimperlé. Je n'ai pu, malgré mes recherches, en retrouver aucun » (Le Men, 1868, p. 366). 


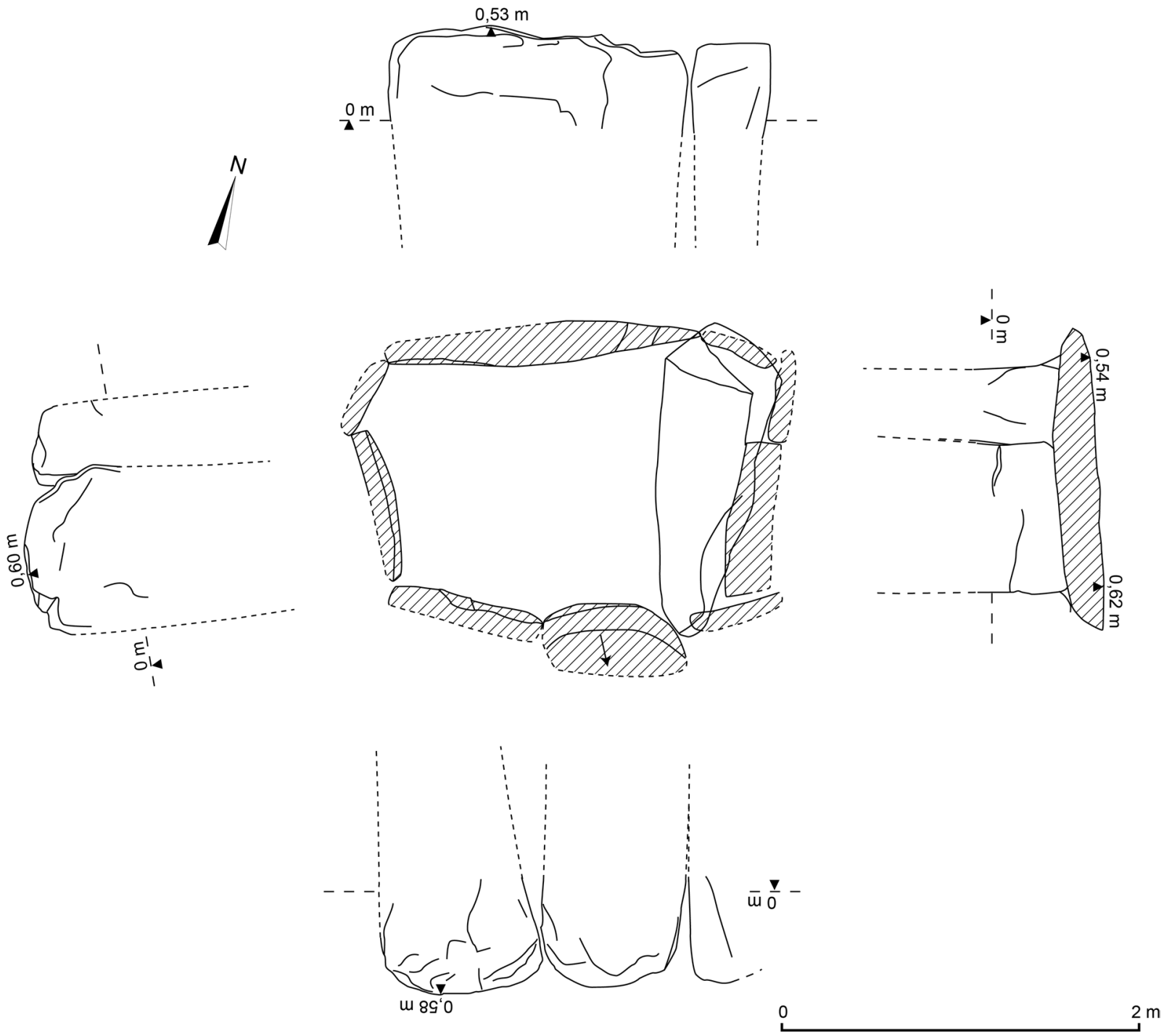

Fig. 10 - Plan et élévations du coffre de Lothéa, Quimperlé, Finistère (DAO : C. Nicolas, Université Paris I; relevé : C. Nicolas, Y. Sparfel, Y. Guéguen).

Plan and elevations of the Lothéa cist, Quimperlé, Finistère (CAD : C. Nicolas, Université Paris I, relevé : C. Nicolas, Y. Sparfel, Y. Guéguen).

\section{LES POIGNARDS EN CUIVRE ARSÉNIÉ} (FIG. 11, FIG. 14, NOS 1 À 4)

Poignard $\mathrm{n}^{\circ} 1$ : longueur : $518 \mathrm{~mm}$; largeur : 93,5 mm ; épaisseur : 4,6 $\mathrm{mm}$; poids : $624,4 \mathrm{~g}$.

Poignard $\mathrm{n}^{\circ} 2$ : longueur : $485 \mathrm{~mm}$; largeur : $99 \mathrm{~mm}$; épaisseur : $3,6 \mathrm{~mm}$; poids $597,5 \mathrm{~g}$.

Poignard $\mathrm{n}^{\circ} 3$ : longueur : $436 \mathrm{~mm}$; largeur : 91,5 mm ; épaisseur : $3,5 \mathrm{~mm}$; poids : 429,7 $\mathrm{g}$.

Poignard $\mathrm{n}^{\circ} 4$ : longueur : $281 \mathrm{~mm}$; largeur : $83 \mathrm{~mm}$; épaisseur : 7,8 $\mathrm{mm}$; poids : $389,8 \mathrm{~g}$.

Les quatre poignards de Lothéa ont la particularité rare de présenter encore l'éclat du métal malgré les millénaires.
Cet éclat a pu être interprété à tort comme une argenture (Lukis, 1846 ; Mortillet, 1903, p. 10). Quelques foyers limités de corrosion verdâtre apparaissent sur les lames et une patine rouge s'est formée sur l'extrémité des poignards $n^{\circ} 1$ et 3 (fig. 11). Une analyse à la microsonde et un examen micrographique ont été réalisés en 1974 par D. Arnaud sur un rivet et un échantillon de la garde du poignard $n^{\circ} 2$ (fig. 14). L'analyse a mis en évidence un alliage composé à 94,50\% de cuivre et $5,10 \%$ d'arsenic pour le rivet et $92,30 \%$ de cuivre et $7,10 \%$ d'arsenic pour le cœur de la lame. La surface de la lame présente un taux d'arsenic beaucoup plus grand avec 69,80\% de cuivre et $29 \%$ d'arsenic. Trois de ces poignards (fig. 14, nos 1 à 3) ainsi que deux de leurs rivets chacun ont été analysés par la suite au laboratoire de Stuttgart (Krause, 2003 ; Tabl. II). 


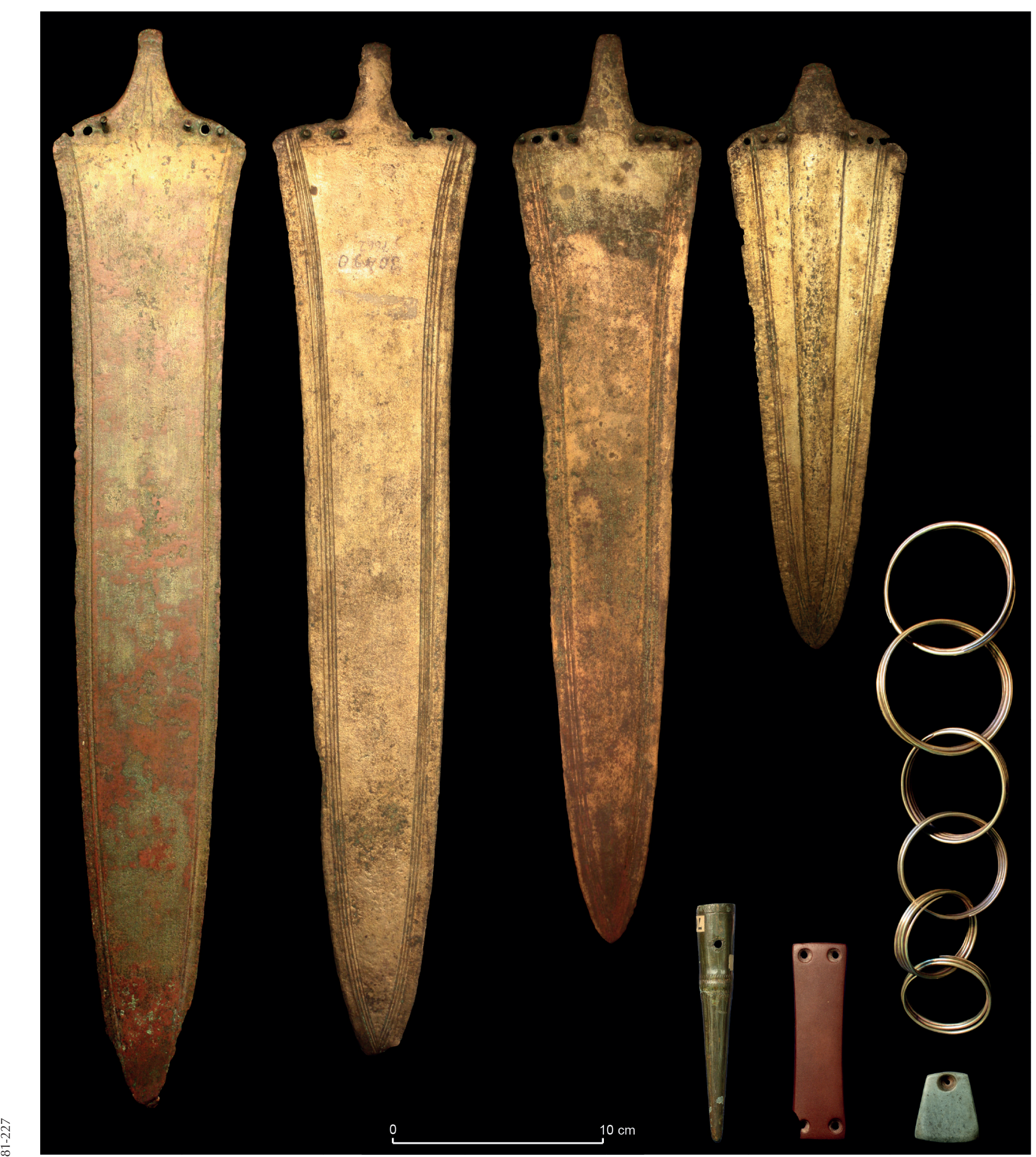

Fig. 11 - Photographies des poignards en cuivre arsénié, du talon en bronze, de la chaîne en or, du brassard d'archer en schiste pourpre et de la hachette-pendeloque en jadéitite de Lothéa (Quimperlé, Finistère), conservés au musée d'Archéologie nationale à Saint-Germain-en-Laye et au musée de Cluny (clichés : C. Nicolas, Université Paris 1).

Photographs of the arsenical copper daggers, the bronze butt, the golden chain, the purple slate wristguard and the jadeitite hatchet-pendant from Lothéa (Quimperlé, Finistère), kept in the National museum in Saint-Germain-en-Laye and the Cluny museum (photographs: C. Nicolas, Université Paris 1). 
Les résultats font tous apparaître des taux d'arsenic importants. Toutefois, concernant le poignard $\mathrm{n}^{\circ} 2$, les taux sont plus faibles pour la lame : $5 \%$ contre $7,1 \%$ de même que pour le rivet : $2,9 \%$ contre $5,10 \%$ (mais les méthodes d'analyse divergent : microsonde d'un côté, spectrographie de l'autre). On peut noter qu'un des rivets du poignard $n^{\circ} 3$ (Tabl. II, analyse 21832) ne comporte que peu d'arsenic $(0,64 \%$, soit le taux le plus faible et de loin), mais par contre un taux d'étain déjà assez conséquent $(0,8 \%)$ alors que cet élément est totalement absent des autres analyses, s'agit-il déjà d'une tentative de substitution de fondant? Il est à l'heure actuelle impossible de se prononcer.

L'examen micrographique a permis d'illustrer la composition biphasée de la lame illustrant une surface claire riche en arsenic et une matrice noire présentant un aspect feuilleté dû à un martelage (Briard, Mohen, 1974). D. Arnaud conclut à une adjonction volontaire d'arsenic, confirmant ainsi l'étude d'autres lames du Bronze ancien faite par H.-J. Hundt (1971). Cette hypothèse d'un ajout volontaire a récemment été réétudiée en détail par F. Papillon (1997) : si l'hypothèse d'ajout d'arsenic par cémentation reste toujours envisageable celle d'un enrichissement de surface via le phénomène de ségrégation inverse, fortement probable dans le cas des cuivres arséniés, ne peut être mise de côté (Papillon, 1997, p. 42). La corrosion très limitée et la brillance remarquable du métal ont été observées sur d'autres poignards en cuivre arsénié (Hundt, 1971 ; Roussot-Laroque, 2008), cela s'explique vraisemblablement par le taux important d'arsenic.

La conservation exceptionnelle des poignards permet de faire quelques observations technologiques concordant avec l'analyse métallographique. Le poignard $n^{\circ} 2$, le mieux conservé, présente des groupes de stries composées d'une ou deux grandes stries d'un centimètre de long et d'une quinzaine de stries perpendiculaires plus courtes (fig. 13, $\mathrm{n}^{\circ} 2$ ). Ces groupes de stries, marquant nettement le métal, se retrouvent sur toute la surface de manière répétée et se recoupent entre eux. On pourrait y voir les traces du martelage mis en évidence par la micrographie. Un autre type de stries est observable au niveau de la patine rouge des poignards. Il s'agit de fines stries longues et parallèles orientées dans le sens de la lame (fig. 13, $n^{\circ} 3$ ). Deux interprétations peuvent être proposées. Ces stries pourraient correspondre à une ultime phase de lissage lors du probable enrichissement de la surface en arsenic. Cependant, on ne peut exclure que ces stries soient dues à la conservation des poignards dans des fourreaux. En effet comme l'attestent certaines découvertes, ces poignards étaient rangés dans des fourreaux avec une doublure intérieure en peau non épilée (Balquet, 2001). Les poils de la doublure pourraient également avoir strié la lame à force de la dégainer et rengainer.

Les décors de filets parallèles aux bords sont d'exécutions variées. Un seul large filet orne le poignard $n^{\circ} 1$, deux filets les poignards $n^{\circ} 3$ et 4 , et trois filets le poignard $n^{\circ} 2$. Les filets du poignard $n^{\circ} 2$ sont d'une grande régularité (fig. 13, $n^{\circ} 2$ ). Ceux du poignard $n^{\circ} 3$ sont plus irréguliers et montrent un certain nombre de maladresses d'exécution au niveau de la pointe, les filets étant à cet endroit au nombre de trois ou de quatre (fig. 13, $\mathrm{n}^{\circ} 4$ ).

Les quatre poignards présentent une lunule au centre de la garde, ce qui est classique des poignards armoricains de l'Âge du Bronze ancien (fig. 14, nos 1 à 4). Ces lunules, généralement observées par une corrosion différentielle, sont ici clairement imputables à la forme du manche. L'aspect assombri de la garde et de la languette des poignards trahit la présence du manche en matière organique (fig. 11). L'épaisseur de la garde des poignées est donnée par la taille des rivets et ne devait pas dépasser les 17 millimètres. Les rivets de taille décroissante, du centre vers les bords, sont encore mobiles pour la plupart, à l'exception de quelques-uns qui sont soudés par la corrosion. Le martelage à leurs extrémités se lit clairement par la présence de faces d'écrasement sur les têtes des tiges (fig. 13, $\mathrm{n}^{\circ} 5$ ).

Les traces d'utilisation de ces poignards sont assez ténues. Quelques bosselures et de rares entailles sont susceptibles de témoigner de chocs avec une autre arme en métal ou des objets contondants. Une entaille de trois millimètres de longueur près de la garde du petit poignard (fig. $13, \mathrm{n}^{\circ} 6$ ) évoque sans ambiguïté une frappe de taille contre une autre lame (Quilliec, 2007). Deux poignards ont leurs pointes cassées (fig. 14, $\mathrm{n}^{\circ} 1$ et 2). L'origine de la cassure de la pointe du poignard $\mathrm{n}^{\circ} 1$ est d'interprétation difficile (choc, corrosion ?). Celle du poignard $n^{\circ} 2$ résulte d'une cassure par flexion, perceptible par une légère torsion de l'actuelle extrémité. Enfin, ces poignards étaient manifestement rangés dans des fourreaux, comme c'est généralement le cas (Balquet, 2001). Tout comme pour l'emmanchement, une coloration différentielle suggère la présence d'un fourreau pour trois des poignards (fig. 11, fig. $14, n^{\circ} 1,2$ et 4 ). Cela est particulièrement évident pour le plus grand, avec une ligne noirâtre nette qui se situe à un centimètre sous la lunule du manche (fig. 14, $\mathrm{n}^{\circ} 1$ ).

\section{LE TALON EN BRONZE (FIG. 11, FIG. 14, $\mathrm{N}^{0}$ )}

Longueur : 113,5 mm ; largeur : $19 \mathrm{~mm}$; diamètre : $17,6 \mathrm{~mm}$; épaisseur de la paroi : $1,9 \mathrm{~mm}$; poids : 46,8 g.

Décrit lors de sa découverte comme une «pique oxydée » (tabl. I), cet objet ne présente pas de pointe acérée qui justifierait une telle appellation. Il semble bien avoir orné l'extrémité d'une hampe, sans que l'on puisse dire qu'il s'agisse d'un « talon de lance » (Briard, Mohen, 1974). Sa patine lisse de teinte vert kaki se différencie nettement des poignards en cuivre arsénié. L'analyse spectrographique menée par JeanRoger Bourhis montre que ce talon est composé à $83,8 \%$ de cuivre et 13,2 \% d'étain (Briard, Mohen, 1974, p. 49 ; Tabl. II). Le volume creux et la présence de deux trous semblent indiquer que ce talon a été obtenu par fonte à la cire perdue. Ce procédé est connu pour les poignards à poignée en bronze de l'Âge du Bronze ancien continental, mieux connus en France sous le nom de poignard rhodaniens. Un exemplaire est connu en Bretagne, il provient du tumulus de Saint-Fiacre à Melrand (Morbihan; Aveneau de la Grancière, 1898 ; Needham, 2000a). Le procédé de cire perdue des poignées en bronze consiste en la réalisation d'un modèle en cire moulé sur un noyau d'argile maintenu par des tiges, celles-ci sont à l'origine des deux trous (Schwenzer, 2009). Le talon est orné par des incisions en arêtes de poisson sous l'embouchure et au niveau d'un léger renflement médian. Un cercle en pointillés en creux vient souligner les deux perforations. 


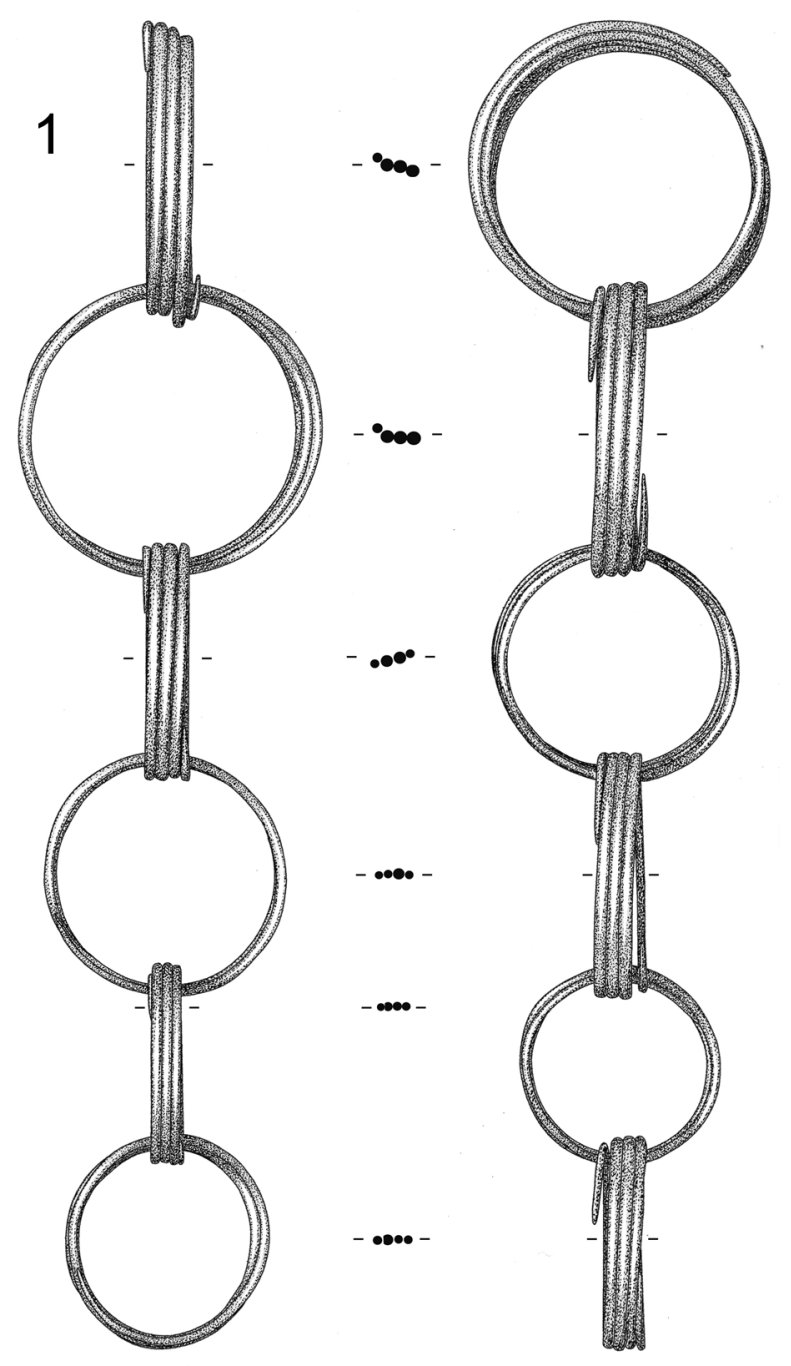

\section{2}

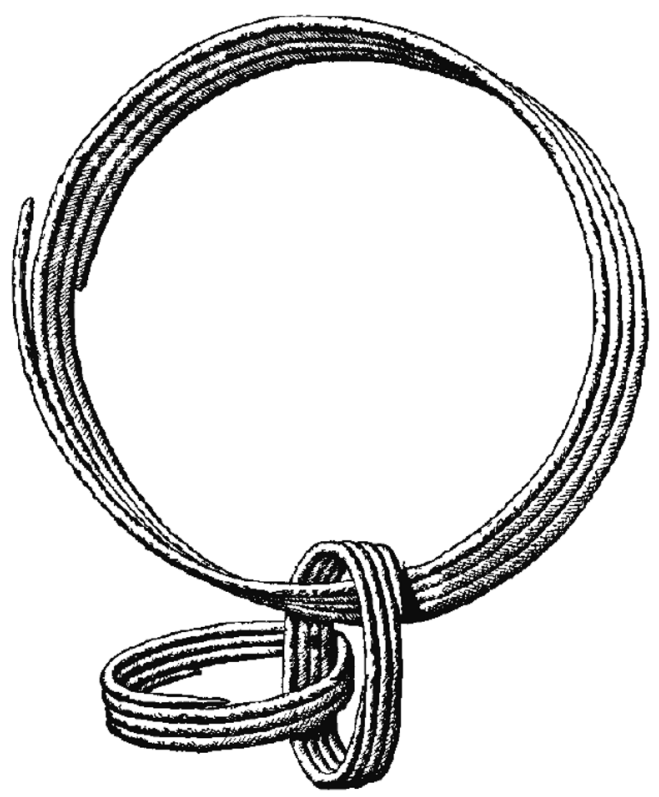

0

$10 \mathrm{~cm}$

Fig. 12 - Chaînes du tumulus de Lothéa, Quimperlé, Finistère. N 1 : chaîne en or (dessin : C. Nicolas, Université Paris 1); $n^{\circ} 2$ : chaîne en argent (d'après Mortillet, 1903, fig. 6).

Chains from the Lothéa barrow, Quimperlé, Finistère. $N^{\circ} 1$ : golden chain (drawing: C. Nicolas, Université Paris 1$) ; n^{\circ} 2:$ silver chain (after Mortillet, 1903, fig. 6).

\section{La « BAGUetTe en CUIVRe » (Fig. 14, $\left.\mathrm{N}^{\circ} 6\right)$}

Un unique dessin de cette baguette existe (Taylor et al., 1846). La seule observation que l'on puisse en retirer est que cette baguette semble avoir une section carrée. Des objets similaires, interprétés comme des lingots, sont connus régionalement en contexte Bronze ancien sur le site du Nouveau Cimetière à Locmariaquer (Morbihan ; Tinevez, 2007) et en contexte Bronze moyen à Mez-Notariou sur l'île d'Ouessant (Finistère ; Le Bihan, Villard, 2010).

\section{LA « HACHE EN BRONZE » (FIG. 14, Nº 7 )}

Il n'est pas parfaitement assuré que cette hache provienne de la tombe de Lothéa (cf. supra). Le dessin publié en 1846 suggère de légers rebords sur les longs côtés, ce qui serait parfaitement cohérent avec les haches à légers rebords découvertes dans les Tumulus de l'Âge du Bronze ancien (Briard,
1984). La nature du matériau, du bronze en l'occurrence, est connue par la seule description de René-François Le Men (1868). En raison de la coexistence de bronze à l'étain et de cuivre arsénié au Bronze ancien, il serait plus convenable, en l'absence d'analyse, de parler d'une hache en alliage cuivreux. Cependant la majorité des haches issues des tumulus armoricains qui ont été analysées se sont révélées contenir de l'étain (Gandois, 2008). Contrairement aux autres mobiliers métalliques de la tombe, cette hache semble avoir subi une corrosion importante de son tranchant et de son talon.

\section{Les POINTES de flèches en SILeX (FIG. 15, $\mathrm{N}^{\text {os }} 3$ À 5)}

Au nombre de sept (tabl. I), ces pointes de flèches en silex sont connues par seulement trois dessins (fig. 15, ${ }^{\text {os }} 3$ à 5). Il s'agit de pointes triangulaires à bords droits $\left(n^{\circ} 3\right.$ et 5 ) ou légèrement convexes $\left(n^{\circ} 4\right)$. Le pédoncule est appointé et les ailerons équarris. 
Tabl. II - Analyses métalliques des objets de Lothéa et de deux poignards du Portugal (analyse de Rennes d'après Briard, Mohen, 1974, analyses de Stuttgart d'après Krause, 2003).

Metal analysis of Lothéa objects and two daggers from Portugal (analysis from Rennes after Briard, Mohen, 1974, analysis from Stuttgart after Krause, 2003).

\begin{tabular}{|c|c|c|c|c|c|c|c|c|c|c|c|c|}
\hline $\mathrm{N}^{\circ}$ d'analyse & Site & Objet & $\mathrm{Sn}$ & $\mathrm{Pb}$ & As & $\mathrm{Sb}$ & $\mathrm{Ag}$ & $\mathrm{Ni}$ & $\mathrm{Bi}$ & $\mathrm{Zn}$ & $\mathrm{Fe}$ & $\mathrm{N}^{\circ} \mathrm{Fig}$. \\
\hline $\begin{array}{l}\text { Stuttgart } \\
21828\end{array}$ & $\begin{array}{l}\text { Lothéa, } \\
\text { Quimperlé, } \\
\text { Finistère }\end{array}$ & $\begin{array}{l}\text { Poignard } \\
n^{\circ} 1, \text { au } \\
\text { niveau de la } \\
\text { garde }\end{array}$ & 0 & 0 & 3,1 & 0,06 & 0,01 & 0,02 & 0,01 & 0 & 0 & $\begin{array}{l}14 \\
n^{\circ} 1\end{array}$ \\
\hline $\begin{array}{l}\text { Stuttgart } \\
21829\end{array}$ & $\begin{array}{l}\text { Lothéa, } \\
\text { Quimperlé, } \\
\text { Finistère }\end{array}$ & $\begin{array}{l}\text { Poignard } \\
n^{\circ} 1 \text {, rivet }\end{array}$ & 0 & 0,01 & 2,8 & 0,02 & 0,01 & 0,02 & 0 & 0 & 0 & $\begin{array}{c}14 \\
n^{\circ} 1\end{array}$ \\
\hline $\begin{array}{l}\text { Stuttgart } \\
21830\end{array}$ & $\begin{array}{l}\text { Lothéa, } \\
\text { Quimperlé, } \\
\text { Finistère }\end{array}$ & $\begin{array}{l}\text { Poignard } \\
n^{\circ} 1 \text {, rivet }\end{array}$ & 0 & 0 & 4,1 & 0,03 & 0,01 & 0,02 & 0,01 & 0 & 0 & $\begin{array}{c}14 \\
n^{0} 1\end{array}$ \\
\hline $\begin{array}{l}\text { Stuttgart } \\
21831\end{array}$ & $\begin{array}{l}\text { Lothéa, } \\
\text { Quimperlé, } \\
\text { Finistère }\end{array}$ & $\begin{array}{c}\text { Poignard } \\
n^{\circ} 3, \\
\text { languette }\end{array}$ & 0 & 0,01 & 2,6 & 0,07 & 0,01 & 0,05 & 0 & 0 & 0 & $\begin{array}{l}14 \\
n^{\circ} 3\end{array}$ \\
\hline $\begin{array}{l}\text { Stuttgart } \\
21832\end{array}$ & $\begin{array}{l}\text { Lothéa, } \\
\text { Quimperlé, } \\
\text { Finistère }\end{array}$ & $\begin{array}{l}\text { Poignard } \\
n^{\circ} 3 \text {, rivet }\end{array}$ & 0,8 & 0 & 0,64 & 0,03 & 0,04 & 0,01 & 0 & 0 & 0 & $\begin{array}{l}14 \\
n^{\circ} 3\end{array}$ \\
\hline $\begin{array}{l}\text { Stuttgart } \\
21833\end{array}$ & $\begin{array}{l}\text { Lothéa, } \\
\text { Quimperlé, } \\
\text { Finistère }\end{array}$ & $\begin{array}{l}\text { Poignard } \\
n^{\circ} 3 \text {, rivet }\end{array}$ & 0 & 0,02 & 3 & 0,06 & 0,01 & 0,03 & 0,01 & 0 & 0 & $\begin{array}{l}14 \\
n^{\circ} 3\end{array}$ \\
\hline $\begin{array}{l}\text { Stuttgart } \\
21834\end{array}$ & $\begin{array}{l}\text { Lothéa, } \\
\text { Quimperlé, } \\
\text { Finistère }\end{array}$ & $\begin{array}{c}\text { Poignard } \\
\mathrm{n}^{\circ} 2, \text { au } \\
\text { niveau de la } \\
\text { garde }\end{array}$ & 0 & 0,03 & 5 & 0,07 & 0,01 & 0,03 & 0 & 0 & 0 & $\begin{array}{l}14 \\
n^{\circ} 2\end{array}$ \\
\hline $\begin{array}{l}\text { Stuttgart } \\
21835\end{array}$ & $\begin{array}{l}\text { Lothéa, } \\
\text { Quimperlé, } \\
\text { Finistère }\end{array}$ & $\begin{array}{l}\text { Poignard } \\
n^{\circ} 2 \text {, rivet }\end{array}$ & 0 & 0,03 & 2,4 & 0,01 & 0,13 & 0 & 0 & 0 & 0 & $\begin{array}{c}14 \\
n^{\circ} 2\end{array}$ \\
\hline $\begin{array}{l}\text { Stuttgart } \\
21836\end{array}$ & $\begin{array}{l}\text { Lothéa, } \\
\text { Quimperlé, } \\
\text { Finistère }\end{array}$ & $\begin{array}{l}\text { Poignard } \\
n^{\circ} 2 \text {, rivet }\end{array}$ & 0 & 0,04 & 2,9 & 0,01 & 0,15 & 0 & 0 & 0 & 0 & $\begin{array}{c}14 \\
n^{\circ} 2\end{array}$ \\
\hline $\begin{array}{c}\text { Rennes } \\
1136\end{array}$ & $\begin{array}{l}\text { Lothéa, } \\
\text { Quimperlé, } \\
\text { Finistère }\end{array}$ & Talon & 13,2 & 0,04 & 0,15 & 0 & 0,05 & 0,06 & 0,01 & 0 & 0,02 & $\begin{array}{c}14 \\
n^{\circ} 5\end{array}$ \\
\hline $\begin{array}{l}\text { Stuttgart } \\
1866\end{array}$ & $\begin{array}{l}\text { Pinhal dos } \\
\text { Melos, } \\
\text { Fornos de } \\
\text { Algofres, } \\
\text { Beira Alta, } \\
\text { Portugal }\end{array}$ & Poignard & 0 & 0 & 4,1 & 0,01 & 0,02 & 0 & 0,01 & 0 & 0,01 & $\begin{array}{l}19 \\
n^{\circ} 3\end{array}$ \\
\hline $\begin{array}{l}\text { Stuttgart } \\
1528\end{array}$ & $\begin{array}{c}\text { Quinta } \\
\text { da Agua } \\
\text { Branca, Vila } \\
\text { Nova de } \\
\text { Cerveira, } \\
\text { Minho, } \\
\text { Portugal }\end{array}$ & Poignard & 0 & 0 & 4,3 & 0,01 & 0,05 & 0 & 0,01 & 0 & 0 & $\begin{array}{l}19 \\
n^{\circ} 6\end{array}$ \\
\hline
\end{tabular}

LE BRASSARD D'ARCHER (FIG. 11, FIG. 15, N 1)

Longueur : $94 \mathrm{~mm}$; largeur : 26,5 mm ; épaisseur : $7,7 \mathrm{~mm}$; poids : $34,5 \mathrm{~g}$.

Le brassard d'archer est réalisé dans un schiste de couleur pourpre, dont les affleurements les plus proches se situent au Cap de la Chèvre (Finistère), soit à $80 \mathrm{~km}$ au nord-ouest de Lothéa. La face supérieure du brassard présente une érosion d'un plan de litage du schiste, cela indiquerait l'utilisation d'une plaquette de schiste comme support. L'ensemble des surfaces sont très bien polies ; des stries fines, courtes, groupées et multidirectionnelles peuvent être reliées à ce polissage. Celles-ci sont plus nombreuses sur la face inférieure et témoignent d'un travail un peu moins soigné. Le brassard de forme globalement rectangulaire adopte des bords légèrement convexes sur les petits côtés et concaves sur les grands côtés. Sa section est plan-convexe. Les perforations, au nombre de quatre, sont biconiques et obtenues pour un tiers à partir de la face supérieure pour deux tiers depuis la face inférieure. Un émoussé brillant peu développé est observable au niveau des bords des perforations (fig. $13, n^{\circ} 8$ ) et des arêtes. Il s'explique vraisemblablement par une usure due à l'attache ou au port du brassard. Un débordement limité de l'émoussé et l'absence d'échancrures suggère un degré d'usure limité du brassard (Bonnardin, 2009). Le brassard pouvait être porté directement sur le bras ou plutôt monté comme ornement d'un brassard en cuir (Fokkens et al., 2008). Enfin un des coins a été cassé, anciennement semble-t-il. 

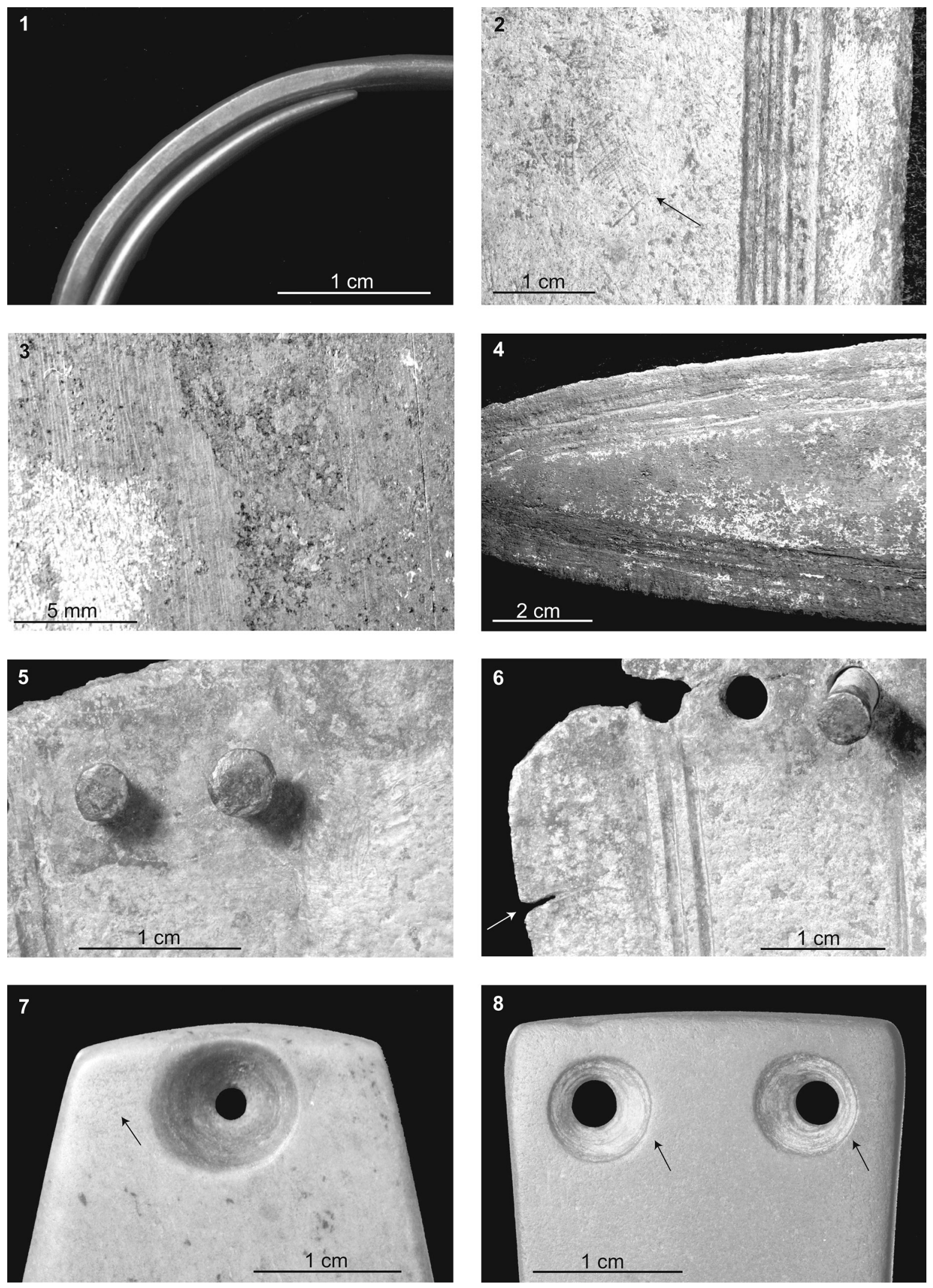
Fig. 13 - Photographies de détail des objets de Lothéa, Quimperlé, Finistère.

$N^{\circ} 1$, détail du fil à section plan-convexe de l'anneau $n^{\circ} 5$ de la chaîne en or; $n^{\circ} 2$, détail de la lame du poignard $n^{\circ} 2$ et d'une possible marque de martelage indiquée par une flèche ; $n^{\circ} 3$, détail de la lame du poignard $n^{\circ} 3$ et des fines stries parallèles observables sur la patine rouge (partie sombre du cliché) ; $n^{\circ} 4$, détail des filets au niveau de la pointe du poignard $n^{\circ} 3 ; n^{\circ} 5$, détail des rivets du poignard $n^{\circ}$ $4 ; n^{\circ} 6$, détail de l'entaille sur le poignard $n^{\circ} 4 ; n^{\circ} 7$, détail du piquetage de la pendeloque en jadéitite ; $n^{\circ} 8$, détail du brassard d'archer, les flèches indiquent les surbrillances au niveau des perforations (clichés: C. Nicolas, Université Paris 1).

Detail photographs of the Lothéa grave goods, Quimperlé, Finistère.

$N^{\circ} 1$, Detail of the wire with plano-convex section of the golden chain ring $n^{\circ} 5 ; n^{\circ} 2$, detail of the dagger $n^{\circ} 2$ blade with possible hammering marks, indicated by an arrow; $n^{\circ} 3$, detail of the dagger $n^{\circ} 3$ blade with fine streaks seen on the red patina (dark side of the photograph) made by polishing or sheath wears; $n^{\circ} 4$, detail of the grooves at the level of the dagger $n^{\circ} 3$ point; $n^{\circ} 5$, detail of the dagger $n^{\circ} 4$ rivets; $n^{\circ} 6$, detail of the cut on the dagger $n^{\circ} 4 ; n^{\circ} 7$, detail of the pecking of the jadeitite hatchet-pendant; $n^{\circ} 8$, detail of the wristguard, the arrows indicates the shines at the level of the level of the holes (photographs: C. Nicolas, Université Paris 1).

\section{LA HACHETTE-PENDELOQUE EN JADÉITITE} (FIG. 11, FIG. 15, $\mathrm{N}^{\circ}$ 2)

Longueur : 33,5 mm; largeur : $31 \mathrm{~mm}$; épaisseur : 8,1 mm ; poids : 19,8 gr. ; volume : $6 \mathrm{ml}$; densité : 3,3.

La pendeloque, faite dans une roche vert pâle, a une densité de 3,3, ce qui l'identifie avec certitude comme étant de la jadéitite. La matière première est une jadéitite très fine. Cette dernière d'un vert clair lumineux est légèrement étirée (spots étirés plus clairs ou légèrement jaunes correspondant peut-être à des micas), quelques points vert foncé ça et là, reliques discrètes d'anciens grenats en atoll (auréoles vert moyen discontinues). D’après P. Pétrequin à qui nous avons transmis des photos de l'objet, un seul diagnostic est possible concernant son origine géographique : Porco ou Bulè dans la zone sud du massif du Mont-Viso, à Oncino (Cuneo, Piémont, Italie ; Pétrequin et al., 2012). Cette petite lame a été mise en forme par un piquetage, dont on peut voir les traces imparfaitement polies sur la face inférieure autour de la perforation (fig. 13, $\mathrm{n}^{\circ} 7$ ). Le reste de la pièce est parfaitement poli et d'une luisance remarquable. Le trou de suspension a été obtenu par perforation biconique. La pendeloque de section quadrangulaire adopte la forme d'un trapèze, dont les bords parallèles sont convexes et la plus grande épaisseur en partie distale. Tout comme le brassard d'archer, un léger émoussé brillant de la perforation indique une suspension.

\section{LE « CASSE-TÊTE »}

Décrit par Arthur Boutarel comme « un gros caillou ayant la forme d'une fève » (tabl. I), il n'a pu être retrouvé. Il s'agit d'« un gros galet de quartzite ne présentant aucune trace d'utilisation » (Briard, Mohen, 1974, p. 51).

\section{UNE RÉOUVERTURE DE LA TOMBE DE LOTHÉA?}

À première vue le mobilier de Lothéa, apparaît homogène avec une série d'objets issus du travail de la pierre, de métaux précieux et de cuivre arsénié. Une seule note discordante vient ternir ce tableau, le talon en bronze (fig. 11, fig. $4, \mathrm{n}^{\circ} 5$ ). Son taux d'étain (13,2\% ; Tabl. II) évoque plutôt des productions du Bronze moyen (1600-1400 avant notre ère) ou des débuts du Bronze final (1400-800 avant notre ère) (Briard, Mohen, 1974, p. 50), conduisant à dater la tombe au plus tôt d'un Bronze ancien
tardif(Briard, 1984, p. 191). Néanmoins cette datation basse reste en contradiction avec le reste du mobilier, qui, nous le verrons, est attribuable à la fin du Campaniforme et au tout début de l'Âge du Bronze ancien. Dès lors, il est nécessaire de s'interroger sur la nature et l'origine de ce talon en bronze. Nous avançons ici trois hypothèses, sans pouvoir apporter de réponse définitive.

\section{LE TALON EN BRONZE NE PROVIENT PAS DE LA TOMBE DE LOTHÉA. Il RÉSULTE D'UN MÉLANGE DE COLLECTIONS}

Pour : Le talon n'est pas illustré avant la publication de J. Briard et J.-P. Mohen (1974). A. Boutarel ne mentionne pas les décors sur la « pique » et la décrit comme ayant la même composition que les poignards (Lettre du 19 août 1843). La patine lisse et brillante du talon diffère sensiblement des autres objets métalliques de la tombe (fig. 11). Ce dernier étant en bronze à l'étain, il aurait dû normalement être soumis à une forte oxydation, comme c'est le cas, semble-t-il, pour la hache à léger rebords (fig. 3) et d'une manière générale pour les objets en bronze trouvés dans les tumulus bretons de l'Âge du Bronze ancien.

Contre : Des éléments ont été attribués à tort à la tombe de Lothéa. Bien que des artefacts aient été perdus au musée d'Archéologie nationale - où le talon est conservé (tabl. I) -, aucune substitution d'objets n'a pour l'heure été mise en évidence.

\section{Le talon en bronze date du Bronze moyen/final, mais SA POSITION DANS LA TOMBE EST INTRUSIVE}

Pour : Les taux d'étain au début de l'Âge du Bronze en Bretagne sont très faibles, 6,39 \% en moyenne (Gandois, 2008). Un cas d'infiltration a été observé dans la tombe du Bronze ancien de Coatanéa (Bourg-Blanc, Finistère), où ont été recueillis, lors du tamisage, une hache à douille armoricaine et un vieux clou en fer (Giot, 1953). Dans ce cas également la tombe était pourtant recouverte d'une dalle de couverture et d'un tumulus.

Contre : Vu la masse du cairn, ce talon peut difficilement provenir de terres infiltrées. Seule une réouverture de la tombe aurait permis de glisser ce talon en bronze, mais l'opération n'aurait alors laissé ni trace dans la structure du tumulus ni remaniement dans la tombe. En effet, l'enquête a posteriori de René-François Le Men présente un mobilier qui semble parfaitement en place (Le Men, 1868, p. 366). 

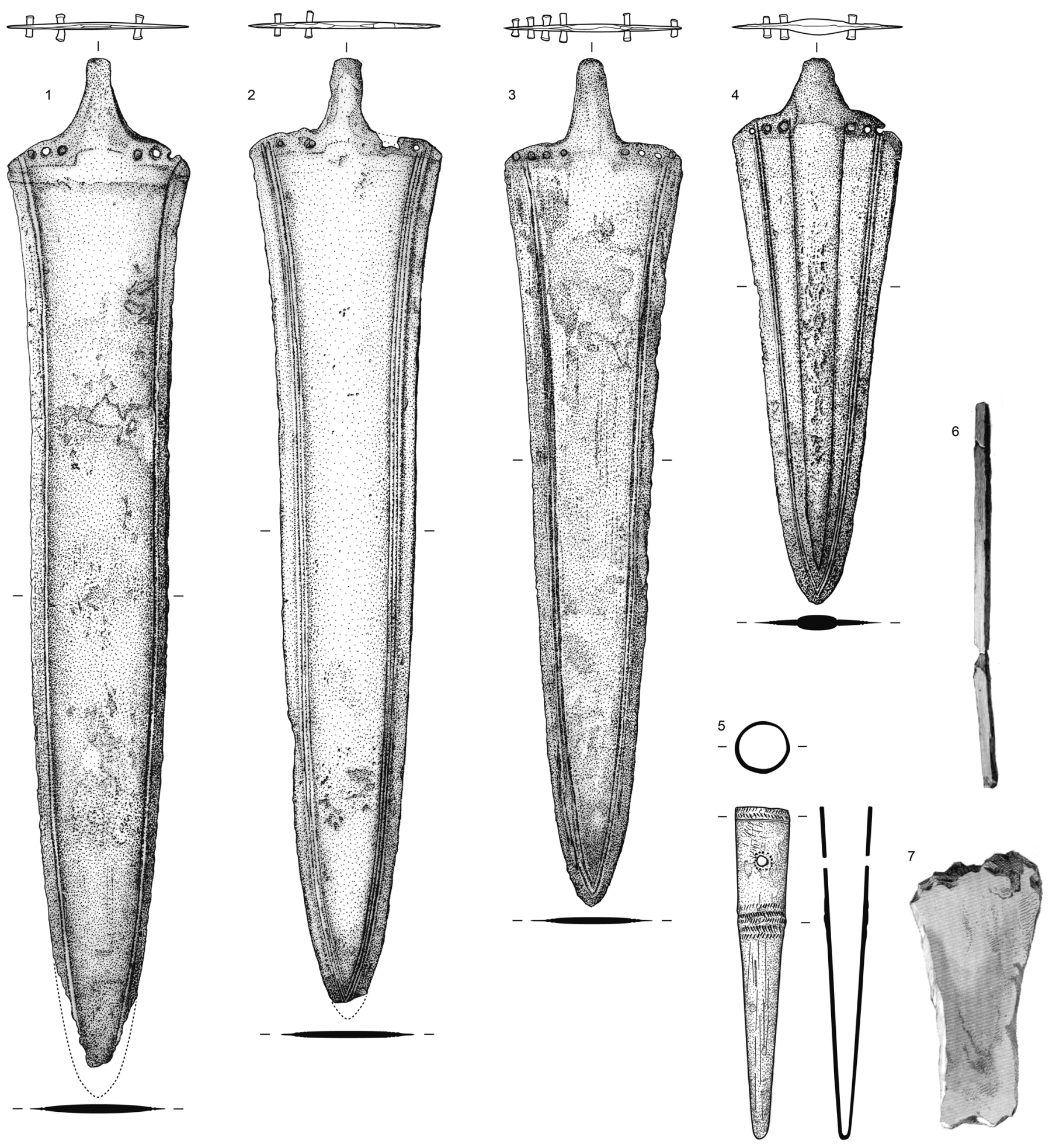

0 $10 \mathrm{~cm}$

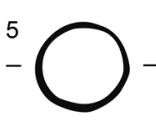

№ 5 à 7

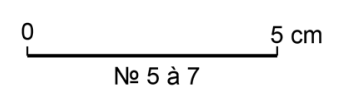

Fig. 14 - Objets à base de cuivre du tumulus de Lothéa, Quimperlé, Finistère. Nos 1 à 3, poignards type Quimperlé en cuivre arsénié ; $n^{\circ} 4$, poignard type Trévérec en cuivre arsénié; $n^{\circ} 5$, talon en bronze; $n^{\circ} 6$, " petite baguette en cuivre "; $n^{\circ} 7$, " hache en bronze » ( $n^{\circ} 1$ à 5 , dessins : C. Nicolas, Université Paris 1, nos 6 et 7, d'après Taylor et al., 1846).

Copper-alloy objects from the Lothéa barrow, Quimperlé, Finistère. Nos 1 à 3, arsenical copper daggers of Quimperlé type ; $n^{\circ} 4$, arsenical copper dagger of Trévérec type ; $n^{\circ} 5$, bronze butt ; $n^{\circ} 6$, " little copper stick "; $n^{\circ} 7$, " bronze axe " ( ${ }^{\circ s} 1$ à 5 , drawings: $C$. Nicolas, Université Paris 1 , $n^{\circ s} 6$ and 7 , after Taylor et al., 1846). 


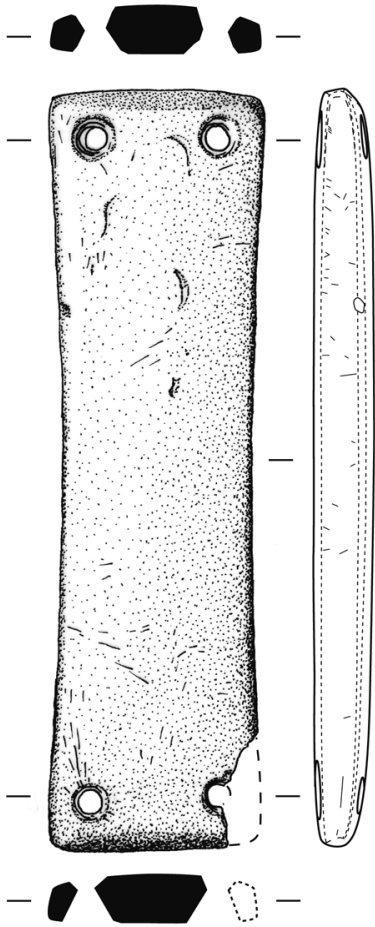

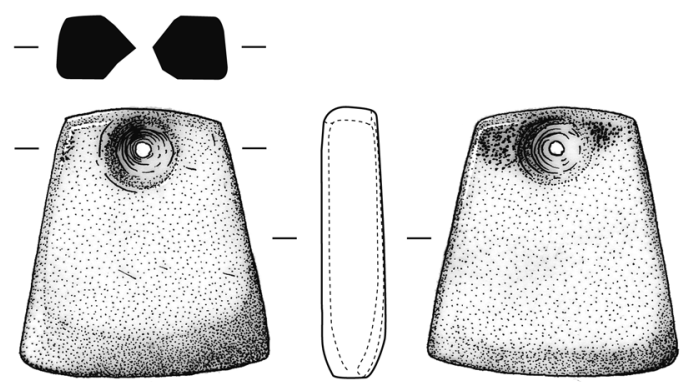
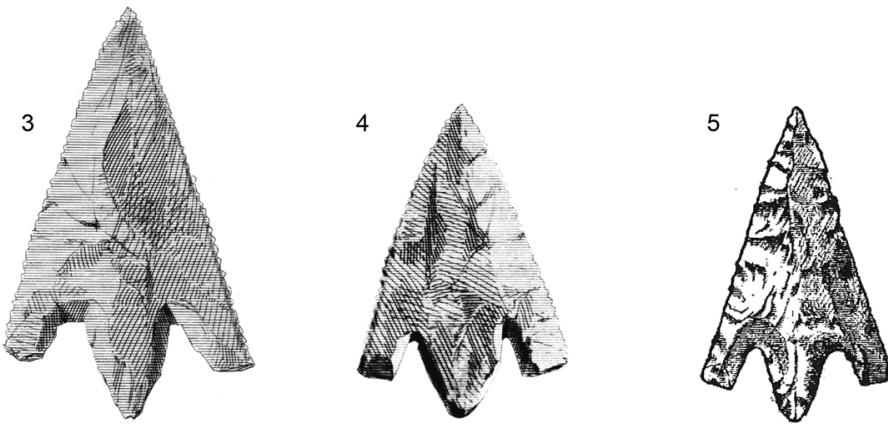

$5 \mathrm{~cm}$

\begin{abstract}
Fig. 15 - Objets en pierre du tumulus de Lothéa, Quimperlé, Finistère. $N^{\circ} \quad 1$, brassard d'archer en schiste rouge; $n^{\circ} 2$, hachette-pendeloque en jadéitite; $n^{o s} 3$ à 5 , pointes de flèches en silex ( $n^{o s} 1$ et 2, dessins : C. Nicolas, Université Paris 1, $n^{\text {os }} 3$ et 4, d'après Taylor et al., 1846, $n^{\circ} 5$, d'après Bertrand, 1891,).
\end{abstract}

Stone objects from Lothéa barrow, Quimperlé, Finistère. $N^{\circ} 1$, purple slate wristguard; $n^{\circ} 2$, jadeitite hatchet-pendant; $n^{o s} 3$ to 5 , flint arrowheads ( $n^{o s} 1$ and 2, drawings: C. Nicolas, Université Paris 1, $n^{o s} 3$ and 4, after Taylor et al., 1846; $n^{\circ} 5$, after Bertrand, 1891).

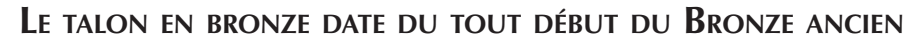
ET IL EST CONTEMPORAIN DU RESTE DU MOBILIER

Pour : Des poignards très bien conservés d'une phase avancée de l'Âge du Bronze ancien (1950-1600 avant notre ère) révèlent des taux d'étain pouvant atteindre les $16 \%$ (Butler, Waterbolk, 1974). La technique de fabrication du talon (fonte à la cire perdue) est attestée à la même époque dans le domaine alpin (Schwenzer, 2009). Les décors du talon se retrouvent sur les céramiques de l'Âge du Bronze ancien (Briard, 1983) et sur les manches des poignards de la même époque (Briard, 1970a). Le talon, unique à notre connaissance, serait un objet original pour la Bretagne par les procédés techniques mis en œuvre (fort taux d'étain, fonte à la cire perdue).

Contre : Il est possible de faire remonter la datation du talon en bronze à une phase avancée du Bronze ancien, mais un décalage chronologique persiste avec le reste du mobilier attribuable au Campaniforme (2500-2150 avant notre ère) ou au début du Bronze ancien (2150-1950 avant notre ère).
Formellement ce type d'objet n'est pas connu avant le Bronze final. Les teneurs de certains éléments trace sont très différentes entre les résultats des poignards et des rivets de Lothéa et celui du talon, principalement pour le bismuth et le fer (Tabl. I).

\section{LOTHÉA DANS SON CONTEXTE RÉGIONAL}

\section{LE MOBILIER EN PIERRE DE LA FIN DU NÉOLITHIQUE ET DU CAMPANIFORME}

La présence d'une hachette-pendeloque en jadéitite pourrait paraître anachronique dans un contexte du Bronze ancien. Ce type de parure en roche rare (roches alpines et fibrolites), de forme rectangulaire ou trapézoïdale, est bien connu en contexte funéraire du Néolithique final. À l'échelle régionale, on en connaît dans les allées couvertes du Kernic en Plouescat (Finistère), de Kersidal en Plomeur (Finistère), la sépulture à entrée latérale de Beaumont à Saint-Laurent-sur-Oust 
(Morbihan ; Tinevez, 1988). Plusieurs de ces monuments ont fait l'objet de dépôts funéraires au Campaniforme mais il est difficile dans la majorité des cas de dire, lorsque pendeloque il y a, si elle appartient à l'une ou l'autre occupation. À Plozévet (Finistère), P. du Chatellier (1883b) signale une pendeloque ovalaire en jadéite dans le dolmen de Penker - possible allée couverte ruinée - associée à du mobilier campaniforme comprenant un gobelet de type maritime, un poignard en cuivre, un brassard d'archer, un V-bouton en os. J. L'Helgouach (1965, p. 285) signale une pendeloque en jadéitite dans l'allée couverte de Kermeur-Bihan (Moëlan-sur-Mer, Finistère) associée là-aussi à du mobilier céramique campaniforme et à des pointes de flèche à pédoncule et ailerons équarris. La nouveauté morphologique par rapport au Néolithique final réside peut-être dans la présence d'un méplat au talon et à l'extrémité la plus large (qui correspondrait pour une hachette classique au tranchant; fig. $15, \mathrm{n}^{\circ} 2$ ). Ce ne sont pas moins de huit parures similaires en ambre qui ont été recueillies dans la tombe du Bronze ancien de Kernonen en Plouvorn (Finistère ; Briard, 1970a et b).

Toutefois, les objets en pierre polie ne sont pas inconnus des viatiques funéraires des tombes de l'Âge du Bronze. En témoigne la présence dans le tumulus Bronze ancien de SaintJude à Bourbriac (Côtes d'Armor) d'un fragment de petite hache polie trapézoïdale en fibrolite qui avait été déposée sur les assises supérieures du bord intérieur du cairn central (Briard et al., 1977a). À Brownstone Farm à Kingswear (Devon, Grande-Bretagne), un ciseau en jadéitite a été découvert associé à des os brûlés et des tessons de céramique dans un coffre sous un tumulus circulaire (Rogers, 1947). Un des os a fait récemment l'objet d'une datation 14C et a donné le résultat suivant : $3420 \pm 30$ BP (SUERC-21367), soit 1870-1630 calBC à $2 \sigma$, soit une date en plein Âge du Bronze ancien (Sheridan, Pailler, 2012). Plus près de la Bretagne, c'est un ciseau en jadéitite, identique par la forme et la matière, qui a été mis au jour par François-Jean Verger en 1848 dans la tombe à couloir de la Motte à Pornic (Loire-Atlantique ; M.-H. Santrot, communication personnelle ?). Dans la même sépulture, Pitre de Lisle du Dréneuc (1892) a mis au jour, une trentaine d'années plus tard, un gobelet et une armature campaniformes, une flèche armoricaine, une alène en cuivre arsénié et douze perles tubulaires en or. Il est donc possible qu'il s'agisse d'un autre cas de réemploi d'un objet ancien en roche alpine à l'âge du Bronze ancien.

Les hachettes-pendeloques sont connues en contexte bien daté dans le nord de la France, où elles semblent nombreuses entre c 3200 et 2800 avant notre ère (Bailloud, 1964). Dans les villages littoraux du Jura français, de petites haches de travail non-perforées en roches alpines sont encore utilisées jusqu'aux environs de 2700 ans avant notre ère (Jeudy et al., 1997). Le même phénomène a été observé dans le Midi de la France à la même époque (Bordreuil et al., 2008). Depuis l'arc alpin, elles auraient rejoint le Bassin parisien, puis la péninsule armoricaine, où certaines d'entre elles sont détournées de leur fonction initiale pour être portées en pendeloque. Dans le cas de la parure de Lothéa, la récupération d'un objet plus ancien est plausible, celui-ci ayant pu faire l'objet d'un refaçonnage (méplats du talon et de la partie active).

Le brassard d'archer de Lothéa est un des rares de Bretagne à présenter quatre perforations, les autres brassards présentant généralement deux perforations (Briard, L'Helgouach, 1957 ; Sangmeister, 1964 ; Treinen, 1970 ; Riquet et al., 1963 ; fig. 16). Il existe probablement un second brassard d'archer à quatre trous, trouvé dans une « chambre sépulcrale », connu par une mention ancienne : « une pierre polie rectangulaire, longue de 10 à 12 centimètres, et percée d'un trou à chacun de ses angles » (Le Men 1877, p. 133). Ces brassards bretons, réalisés généralement en schiste noir (L'Helgouach, 1995), ou exceptionnellement en métadolérite de type A (Le Roux, 1999), sont à rattacher à la culture campaniforme (Riquet et al., 1963). De formes généralement allongées, leurs sections sont rectangulaires, plan convexes ou biconvexes. Seuls deux brassards présentent, comme celui de Lothéa, de grands bords concaves et un ou deux petits bords convexes. Il s'agit de brassards en schiste noir provenant des sépultures mégalithiques de Kerallant (Saint-Jean-Brévelay, Morbihan ; fig. 16, $\mathrm{n}^{\circ} 14$ ) et de Nelhouët (Caudan, Morbihan). Rappelons ici que le brassard de Lothéa a été façonné dans un schiste pourpre, ce qui le différencie par sa couleur des autres exemplaires armoricains. Quatre brassards sont connus pour l'Âge du Bronze ancien mais ils se distinguent par l'emploi de matières premières précieuses ou exotiques (or, ambre, jais), des dimensions plus réduites et des bords systématiquement concaves (Butler, Waterbolk, 1974 ; Briard, 1970a ; Briard et al., 1994 ; Needham, 2000a).

Les flèches de Lothéa renvoient également à la culture campaniforme. Les armatures à pédoncule et ailerons équarris sont depuis longtemps reconnues comme typiques du Campaniforme (Riquet et al., 1963). Cependant les trois exemplaires connus de Lothéa varient des armatures trapues triangulaires, où pédoncule et ailerons sont équarris. Les flèches de Lothéa sont sensiblement plus allongées et présentent un pédoncule appointé. Deux armatures de flèches identiques ont été trouvées dans un tumulus à Kermenhir (Poullan-sur-Mer, Finistère ; Chatellier, 1907 ; fig. 16, n 8), auxquelles on peut ajouter une armature du tumulus de Fao-Youen (PlonéourLanvern, Finistère) dont un aileron est équarri et l'autre taillé en oblique (Balquet, 2001, p. 101). Deux autres armatures fort semblables ont été mises au jour, une dans l'allée couverte coudée du Luffang (Crac'h, Morbihan ; Le Rouzic, 1898 ; Nicolas, 2012) et l'autre au rocher de Beg-er-Goalennec (Quiberon, Morbihan ; Gaillard, 1906). Le site d'habitat de Penancreac'h (Quimper, Finistère) occupé du Campaniforme au Bronze ancien a lui-aussi livré deux flèches du même type (Le Bihan, 1993 ; Le Bihan et al., 1994). Les prospections ou les découvertes fortuites fournissent également leurs lots d'armatures similaires à celles de Lothéa : une dans la forêt de Carnoët (Annic, 2000), deux en grès lustré à Kervouster (Guengat, Finistère ; Le Goffic, 1996) et à Penhoat-SaintThomas (Pleuven, Finistère ; Le Goffic, 1999), une dernière sur le plateau du Collédic (Saint-Nicolas-du-Pélem, Côtes d'Armor) découverte en même temps qu'une importante série céramique du Campaniforme et de l'Âge du Bronze ancien (Le Provost et al., 1972). Tout porte à considérer les armatures de Lothéa, qui présentent des ailerons équarris d'une part et une forme allongée et un pédoncule appointé d'autre part comme un type intermédiaire entre les armatures campaniformes à pédoncule et ailerons équarris et les flèches armoricaines de l’Âge du Bronze ancien (Nicolas, 2011). 


\section{LES ARMES À BASE DE CUIVRE}

Les poignards en cuivre arsénié et la hache sont des objets très bien connus dans les tombes de l'Âge du Bronze ancien. Les trois grands poignards de Lothéa (fig. 14, n ${ }^{\text {os }} 1$ à 3) ont servi à la définition du type éponyme de Quimperlé, par Stuart Needham, sur les critères de leur grande longueur et de leur section plate (Needham, 2000a, p. 153). Le poignard $n^{\circ} 1$ de Lothéa est le plus grand exemplaire de type Quimperlé, en tenant compte que sa pointe est cassée, celui-ci mesurait 53 centimètres de longueur (fig. 14, $\mathrm{n}^{\circ} 1$ ). De même, Gretel Gallay, dans sa typologie des poignards du Bronze ancien en France, les a distingués sous l'appellation « longs poignards de type Rumédon » (Gallay, 1981, p. 93). Seul Jacques Briard donne une limite entre petits et grands poignards en considérant les lames de plus de $30 \mathrm{~cm}$ comme des épées (Briard, 1984, p. 86). Nous préférons l'appellation de poignards, étant donné la proximité entre petits poignards et grands poignards et l'inexistence à l'Âge du Bronze ancien d'une production de lames allongées et aigües désignables sous le nom d'épée. Quatorze poignards de type Quimperlé sont connus en Bretagne, trouvés presque exclusivement dans les riches tombes à pointes de flèches. Ils sont, à l'exception des trois poignards de Lothéa, toujours trouvés en un seul exemplaire, accompagnés parfois d'autres types de poignards (Needham, 2000a, p. 158 ; Needham, 2000b ; Balquet, 2001). On peut y ajouter une lame conservée au musée du Cabinet des Médailles à Paris (Briard et Mohen, 1974) et une autre trouvée sur les bords de la rivière de l'Aff à Bains-sur-Oust (Ille-et-Vilaine) ; selon Victor Micault (1883, p. 100), cette dernière serait identique à celles de Carnoët. Au regard de la typochronologie de Stuart Needham, ces poignards de type Quimperlé apparaissent parmi les plus anciens poignards de l'Âge du Bronze ancien breton (Needham, 2000a, p. 158). Le taux d'arsenic des poignards de Lothéa est également un élément pour leur relative ancienneté. Le cuivre arsénié est attesté notamment dans la métallurgie du Campaniforme atlantique (Briard et al., 1998) et des débuts de l'Âge du Bronze ancien. Les cuivres ou bronzes arséniés disparaissent assez rapidement au profit de bronze à l'étain au cours du Bronze ancien breton (Gandois, 2011). Le quatrième poignard de Lothéa est, avec son renflement médian, caractéristique du type Trévérec (Gallay, 1981 ; Needham, 2000a ; fig. 14, $\left.n^{\circ} 4\right)$. Ce type est relativement commun en Bretagne (26 exemplaires). Pour Stuart Needham, ce type de poignard apparaitrait dans un second temps au Bronze ancien en Bretagne (Needham, 2000a, p. 158), sans que nous puissions confirmer son point de vue. La hache à légers rebords, avec les réserves émises ci-dessus, est du type communément trouvé dans les tumulus bretons de l'Âge du Bronze ancien (Briard, 1984).

\section{LOTHÉA ET LES TOMBES AUTOUR DE LA LAÏTA}

Le tumulus de Lothéa n'est pas isolé dans son environnement et appartient à une petite concentration de tertres et de coffres mégalithiques répartis autour du fleuve côtier, la Laïta (fig. 1, $\mathrm{n}^{\circ}$ 20). Il occupe une position remarquable, formant le sommet d'une colline s'élevant à 58 m (NGF) de hauteur et dominant une boucle de la Laïta. Plus largement, il s'inscrit dans un paysage funéraire local dense, marqué depuis le Néolithique moyen par des tertres tumulaires et diverses sépultures collectives (Gouézin, 2007).

$\mathrm{Au}$ Campaniforme, une grande partie de ces monuments mégalithiques sont toujours en fonctionnement : réutilisation de tombes à couloir du Néolithique moyen et d'allées couvertes du Néolithique récent/final et de dolmens plus anciens (Salanova, 2003). Dans la sépulture à entrée latérale du Goërem (Gâvres, Morbihan), deux dépôt funéraires campaniformes ont été reconnus, l'un d'eux ayant nécessité l'enlèvement d'une dalle de couverture (L'Helgouach, 1970), tandis que dans la probable sépulture à entrée latérale de Tuchenn-Gouc'h (Ploemeur, Morbihan), le mobilier campaniforme se trouvait dans un petit coffre aménagé dans la galerie principale (Gouézin, 2007, p. 84). Les gobelets campaniformes sont trouvés en abondance dans ces sépultures collectives, de même que les objets généralement associés à cette culture : alène, pointe foliacée à soie et poignard en cuivre, agrafes en or, brassards d'archer et armatures à pédoncule et ailerons équarris ont été découverts associés ou isolés à Kerandrèze (Moëlan-sur-Mer, Finistère ; Chatellier, 1883a), Port-Fétih (Ploemeur, Morbihan ; Gouézin, 2007), au Goërem (Gâvres, Morbihan ; L'Helgouach, 1970) et à Nelhouët (Caudan, Morbihan; Treinen, 1970). La distribution de ces artefacts campaniformes suit celle des sépultures mégalithiques, qui sont situées essentiellement sur le littoral (fig. 1). Dans une moindre mesure à l'Âge du Bronze ancien, l'utilisation des sépultures collectives se poursuit, comme l'atteste le petit vase biconique décoré de chevrons découvert dans la tombe mégalithique de Kercavès (Ploemeur, Morbihan ; Briard, 1983).

L'essentiel des tombes individuelles environnant la Laïta n'a pas livré de mobilier. Leur exploration témoigne parfois de fréquentations durant la période gallo-romaine et de fouilles anciennes (Galliou, 2009). Il nous faut signaler le tumulus de Cruguel à Guidel (Morbihan). Cette tombe a livré quatre poignards, une hache et une tige en bronze, ainsi que quatorze flèches triangulaires de type armoricain (Le Pontois, 1890). Ce tumulus se trouve à $8 \mathrm{~km}$ en aval de celui de Lothéa, près de l'embouchure de la Laïta. La présence de deux tumulus à pointes de flèches richement dotés souligne l'importance au Bronze ancien de ce fleuve côtier, navigable sur près de $15 \mathrm{~km}$ de Quimperlé à son estuaire, ainsi que de son bassin versant. On peut d'ailleurs mentionner que cette concentration de tombes se trouve dans une des zones de Bretagne riches en cassitérite (Guigues, 1970).

Plusieurs tombes des environs de la Laïta présentent des similarités avec la sépulture de Lothéa par leur caractère mégalithique (fig. 1). Elles sont composées de trois à sept dalles de chant coiffées d'une dalle de couverture. Les coffres de Kerouarc'h (Guidel, Morbihan), Roscasquen (Quimperlé, Finistère) et Kerboulou (Le Trévoux, Finistère) sont composés respectivement de trois, cinq et six dalles de chant; à Kerouarc'h et à Kerboulou un tumulus recouvrait la tombe (Briard, 1984). Le dolmen de Lez-Variel (Guidel, Morbihan) forme une petite chambre trapézoïdale fermée par cinq dalles de chant de $1 \mathrm{~m}$ de hauteur, deux dalles viennent doubler le mur à l'est, une seule pierre forme un dallage tandis qu'une grande pierre sert de couverture (Gouézin, 2007, p. 66). La tombe de Kercadoret (Moëlan-sur-Mer, Finistère) est composée de cinq dalles de 
chant formant une chambre grossièrement pentagonale (Le Roux, 1974, 1999). Ces petits coffres mégalithiques fermés sont assez mal datés. Ils ont livré peu de mobilier qui nous soit connu. Deux d'entre eux ont livré un mobilier attribuable à une période entre la fin du Néolithique et l'Âge du Bronze ancien. Le premier est celui de Lez-Variel à Guidel (Morbihan) fouillé par l'abbé Euzenot. L'intérieur du monument n'a pas livré de mobilier, mais en creusant une tranchée d'accès à la tombe, l'abbé Euzenot a mis au jour à un mètre du dolmen un ciseau ou hache « en bronze » et une pierre à cuvette identifiable comme une meule (Euzenot, 1868 et 1878). Ce type de meule est connu dans des contextes allant du Néolithique final à l'Âge du Bronze ancien en Bretagne (Pailler et al., 2010 ; Donnart, 2011), comme dans le Bassin parisien (Brunet et al., 2004). Le coffre mégalithique de Kercadoret a livré pour seul mobilier un fragment de brassard d'archer en métadolérite de type A (Le Roux, 1975, p. 531, 1999, p. 215 ; fig. 16, n 3). Ces quelques indices suggèrent l'édification de tombes individuelles au Campaniforme, et peut-être dès le Néolithique final. Cependant ce corpus trop étroit des rives de la Laïta mérite d'être élargi.

\section{LOTHÉA ET LES SÉPULTURES INDIVIDUELLES CAMPANIFORMES EN BRETAGNE}

\section{RAPPEL HISTORIQUE}

La sépulture individuelle apparaît en Bretagne dès la fin du Mésolithique (5500-5000 avant notre ère), avec les fameuses nécropoles de Téviec et Hoëdic (Morbihan), et adopte des formes monumentales au Néolithique moyen I (4500-4300 avant notre ère) avec les grands tertres allongés, de la région de Carnac notamment (Boujot et Cassen, 1992). Toutefois, certaines tombes, comme celle du Castellic/Bois du Latz (Carnac, Morbihan ; Fontès, 1881), avec coffre mégalithique, cairn et tumulus circulaire, attestent l'existence dès cette époque de la formule architecturale développée à l'Âge du Bronze ancien. La suite du Néolithique est largement dominée par les sépultures collectives (Boujot, Cassen, 1992). Pour l'heure, le retour à la sépulture individuelle ne semble pas apparaître avant la fin du Néolithique. Une ébauche de hache de combat en métahornblendite accompagnée de trois haches polies d'un instrument en quartz et d'un objet en terre cuite a été découverte dans une fosse recouverte d'un petit tertre de six mètres de diamètre et $0,7 \mathrm{~m}$ de hauteur à Kervadel (Plobannalec-Lesconil, Finistère) (Chatellier, 1881, 1907, p. 313). Dans le tertre du dolmen de Mané-Meur (Quiberon, Morbihan), il est signalé la découverte d'« un coffre en pierres avec squelette replié et une hachemarteau naviforme » (Le Rouzic, 1965, p. 52). Ces deux monuments pourraient correspondre à des tombes individuelles sous tumulus datant du Néolithique final (Giot et Cogné, 1955).

Les tombes individuelles du Campaniforme en Bretagne ont longtemps été ignorées, à tel point qu'aucune d'entre elles ne figurent dans le récent inventaire de ces sépultures en France (Salanova, 2011). Seule une hypothétique tombe sans structure associée est signalée à Milin Coz (Plestin-les-grèves, Côtes-d'Armor ; Salanova 2000, 2011). Elle correspond à un gobelet de type épimaritime découvert isolément lors de la destruction d'un talus. Plusieurs raisons expliquent sans doute ce tableau. D'un côté, la nature mégalithique de certaines tombes individuelles a porté au XIX ${ }^{\mathrm{e}}$ siècle à les qualifier de dolmens, sans que les architectures soient bien comprises. Dans le même temps, les gobelets campaniformes ont été retrouvés en grand nombre dans des sépultures collectives du Néolithique. La réutilisation des sépultures collectives par les Campaniformes a été depuis largement mise en évidence (L'Helgouach, 1965, 1970 ; Salanova, 2003). Partant de ce constat, la découverte de gobelets campaniformes dans des dolmens, même fermés, était interprétée comme un réemploi d'un monument plus ancien. Cependant, les fouilles anciennes ou récentes de quelques coffres mégalithiques ont amené les chercheurs régionaux à s'interroger sur l'existence de tombes individuelles construites par les Campaniformes (Chatellier, 1907, p. 46-47 ; Briard, 1989 ; Giot, 1990 ; L'Helgouac'h, 1995 ; Le Roux, 1999 ; Salanova, 2011). D'un autre côté, les découvertes nombreuses de mobilier en bronze dans les tumulus ont conduit à rattacher presque exclusivement ces monuments au Bronze ancien, même si quelques éléments sont rattachés à un Chalcolithique mal défini (Briard, 1984).

\section{INVENTAIRE}

\section{$N^{\circ} 1$ : Bignat, Kérien, Côtes-d'Armor (fig. 16, $n^{\circ} 1$ )}

Le tumulus de Bignat, fouillé par l'abbé Le Foll, a livré un vase épicampaniforme et les restes d'un poignard en « bronze » (Micault, 1883, p. 108 ; Briard, 1984, p. 217).

\section{$N^{\circ} 2$ : Juno-Bella, Berrien, Finistère (fig. 16, $n^{\circ} 2$ )}

La tombe de Juno-Bella a été repérée par P. du Chatellier (1897), puis fouillé par J. Briard (1978). Il s'agit d'un coffre fait de cinq dalles de chant, orienté est-ouest et mesurant $1,7 \times 1,1 \mathrm{~m}$. Ce coffre contenait les vestiges d'un squelette et d'un gobelet à anse dont la carène basse atypique nous semble d'affinité campaniforme.

\section{$N^{\circ} 3$ : Kercadoret, Moëlan-sur-Mer, Finistère (fig. 16, $n^{\circ} 3$ )}

Le coffre mégalithique de Kercadoret, détruit en 1973, a pu faire l'objet d'une fouille de sauvetage par C.-T. Le Roux $(1974,1975)$. Les fosses de calage ont pu être retrouvées et les orthostates, déplacés lors de la destruction, s'y ajustent parfaitement. La dalle de couverture mesure 2,3 $\mathrm{m}$ de longueur pour $2 \mathrm{~m}$ de largeur et 0,3 $\mathrm{m}$ d'épaisseur. L'ensemble dessine un plan à peu près pentagonal délimitant un espace d'environ $1,5 \mathrm{~m}$ de diamètre avec une hauteur d'1,3 m. Le mobilier, remanié, comprenait quelques tessons préhistoriques, gallo-romains et médiévaux, mais surtout un brassard d'archer en métadolérite de type A cassés aux extrémités (Le Roux, 1999). 
$N^{\circ} 4$ : Coffre $P$, nécropole de Lesconil, Plobannalec-Lesconil, Finistère (fig. $16, \mathbf{n}^{\circ} 4$ )

La nécropole de Lesconil à Plobannalec compte une vingtaine de structures mégalithiques, explorées en 1878 par P. Du Chatellier (1883a). Dans le tumulus d'une sépulture mégalithique ruinée $(\mathrm{M})$ se trouvait un coffre périphérique $(\mathrm{P})$. Ce dernier, mesurant $1,8 \times 1,7 \mathrm{~m}$, était composé de quatre dalles de chant et d'un pavage de galets marins. Le mobilier découvert à l'intérieur du coffre comprend les restes d'une poterie rouge très fine, un poignard à soie en cuivre, une pierre ponce et un polissoir en grès. Le mobilier est conservé au musée d'Archéologie nationale. Les vestiges du vase comprennent vingt-six tessons de faible épaisseur $(4 \mathrm{~mm})$ et très fragmentés (inférieurs à $2,5 \mathrm{~cm}$ ), rendant impossible toute reconstitution. Les tessons présentent une surface rouge brillante et ne comportent aucun décor.

\section{$N^{\circ} 5$ : Plobannalec-Lesconil 1, Finistère (fig. 16, $n^{\circ} 5$ )}

Aucun contexte de découverte ne nous est connu pour le coffre de Plobannalec-Lesconil 1, d'où provient un brassard d'archer à deux trous (Briard et L'Helgouac'h, 1957, p. 62). Ce dernier est illustré par Charles Bénard (1929, fig. 47) et par Françoise Treinen (1970, fig. 45).

\section{$N^{\circ} 6$ : Plobannalec-Lesconil 2, Finistère}

Préalablement citée, la " chambre sépulcrale 》 de Plobannalec-Lesconil 2 a livré un probable brassard à quatre trous (Le Men, 1877, p. 133).

\section{$N^{\circ} 7$ : Coatjou-Glas, Plonéis, Finistère (fig. $16, n^{\circ} 7$ )}

Le tumulus de Coatjou-Glas a été fouillé et publié par P. du Chatellier (1887). Le caveau, orienté est-ouest, était composé des murs en pierres sèches coiffés d'une dalle de couverture de $1,9 \mathrm{~m}$ de longueur et $1 \mathrm{~m}$ de largeur. Au fond se trouvait un dallage en pierre recouvert par un plancher en bois de chêne. Le caveau était recouvert d'un cairn et d'un tumulus, celui-ci mesurant $14 \mathrm{~m}$ de diamètre pour $1,5 \mathrm{~m}$ de hauteur. Le mobilier funéraire comprend un grand vase à fond plat, un petit poignard en cuivre percé d'un trou de rivet et décoré d'un filet parallèle aux bords, quatre perles - deux olivaires et deux tubulaires - et un brassard d'archer en schiste, deux pointes de flèches en silex - une à pédoncule et ailerons taillés en oblique et une à pédoncule et ailerons naissants - et quatre pierres polies ovoïdes. Ces dernières, assez mystérieuses, étaient « du poids de 50 à 54 grammes, ouvertes par nous, et qui nous ont donné du bronze » (Chatellier, 1887, p. 51).

\section{$N^{\circ} 8$ : Kermenhir, Poullan-sur-Mer, Finistère (fig. 16, $n^{\circ} 8$ )}

Le tumulus de Kermenhir, fouillé en 1883 par P. du Chatellier, a seulement fait l'objet d'une courte notice (Chatellier, 1907).
Le tumulus mesurait $24 \mathrm{~m}$ de diamètre pour $2 \mathrm{~m}$ de hauteur. Le caveau, recouvert d'un cairn, était constitué d'une dalle de couverture et de murs en pierres sèches. Il était orienté est-ouest et mesurait $2,9 \mathrm{~m}$ de longueur pour $1 \mathrm{~m}$ de largeur. La dotation funéraire comprend deux pointes de flèches, une hache plate en cuivre et une pendeloque en schiste (Chatellier, 1907). Une planche des archives de Paul du Chatellier indique que les restes d'un gobelet campaniforme, un tesson décoré et un grattoir auraient également été découverts dans cette sépulture (fig. $16, \mathrm{n}^{\circ} 8$ ). Un tesson du gobelet campaniforme a pu être étudié par Laure Salanova (1996), celui-ci montre un décor linéaire fait par incision.

\section{$N^{\circ} 9$ : Kerimanton, Quéménéven, Finistère (fig. 16, n 9)}

La tombe de Kerimanton a été découverte par un agriculteur en 1953. Seuls subsistaient quatre dalles de chant, formant un caveau de $1,2 \times 0,6 \times 0,8 \mathrm{~m}$ et orienté SE-NO sud-est/nordouest. Dans la tombe se trouvait un vase en terre rougeâtre sans décor, avec une carène à mi-hauteur, qualifiable d'épicampaniforme (Giot, 1953).

\section{N 10 : Lothéa, Quimperlé, Finistère (fig. 12, 14 et 15)}

\section{$N^{\circ} 11$ : La Guette, Paimpont, Ille-et-Vilaine (fig. 16, $\mathbf{n}^{\circ} 11$ )}

La tombe de la Guette a été découverte en 1982. Elle est orientée nord-sud et mesure environ $1,8 \mathrm{~m}$ de longueur pour 1,4 $\mathrm{m}$ de largeur. Trois dalles de chant forment les parois ouest et est. Les parois nord et sud sont constituées de petites dalles empilées qui devaient formaient des murs appareillés. Le tout est pris dans un petit cairn de $3 \mathrm{~m}$ de diamètre. Le dégagement des pierres au centre de la tombe a permis de mettre au jour quelques tessons campaniformes; l'un d'eux présente un décor " à la roulette " de type maritime (Briard, 1989).

\section{N 12 : Le coffre de Roh Du B, La Chapelle-Neuve, Morbihan (fig. 16, $n^{\circ} 12$ )}

Le coffre de Roh Du B a été mis au jour en 1984, puis fouillé par Philippe Gouézin. Il se trouve au centre d'un tumulus de 9,5 $\mathrm{m}$ de diamètre. La sépulture est orientée est-ouest et mesure $1,8 \times 0,8 \mathrm{~m}$. Elle est composée de trois dalles de chant formant les côtés nord, sud et ouest, et éventuellement d'un mur en pierres sèches à l'est. Au fond se trouvait une dalle de granite sur lequel reposaient des tessons campaniformes et une céramique à décor digité (Briard, 1989). Ces derniers ornés de bandes linéaires se rangent dans le style épimaritime. Deux autres coffres (Roh Du A et C) de construction similaire se trouvent à proximité (Briard, 1989).

\section{$N^{\circ} 13$ : Kerouaren, Plouhinec, Morbihan (fig. 16, $n^{\circ} 13$ )}

Le coffre mégalithique de Kerouaren a été fouillé en 1884 par Félix Gaillard. Il est composé de cinq orthostates - un en place, trois inclinés et un renversé -, d'une dalle de couverture et, au fond, d'un dallage en pierre. Il est orienté 
Fig. 16 - Plans et mobiliers des sépultures individuelles campaniformes de Bretagne. Côtes-d'Armor : $n^{\circ} 1$, Bignat, Kérien (d'après Briard, 1984a) ; Finistère : $n^{\circ} 2$, Juno-Bella, Berrien (d'après Briard, 1978) ; $n^{\circ} 3$, Kercadoret, plan des fosses de calage et reconstitution du domen,, Moëlan-sur-Mer (d'après Le Roux, 1974) ; $n^{\circ} 4$, Lesconil, Plobannalec-Lesconil (plan d'après Chatellier, 1883a ; dessins : C. Nicolas, Université de Paris 1) ; $n^{\circ} 5$, Plobannalec-Lesconil (dessin : C. Nicolas, Université de Paris 1) ; $n^{\circ} 7$, Coatjou-Glas, Plonéis (plan et dessins du poignard, des perles et du galet d'après Chatellier, archives, dessins des flèches, du vase et du brassard C. Nicolas, Université Paris 1) ; $n^{\circ} 8$, Kermenhir, Poullan-sur-Mer (d'après Chatellier, archives) ; $n^{\circ} 9$, Kérimanton, Quéménéven, Finistère (d'après Briard, 1984a).

Plans and graves goods of individual Bell Beakers burials from Brittany. Côtes-d'Armor : $n^{\circ} 1$, Kérien (after Briard, 1984a) ; Finistère :

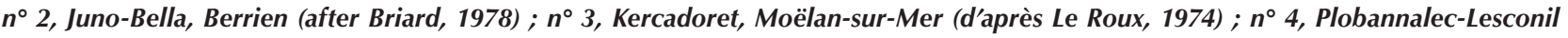
(plan after Chatellier, 1883a, drawings: C. Nicolas, Université de Paris 1) ; $n^{\circ}$ 5, Plobannalec-Lesconil (dessin: C. Nicolas, Université de Paris 1) ; $n^{\circ} 7$, Coatjou-Glas barrow, Plonéis (plan and drawings of the dagger, beads and pebble after Chatellier, archives, drawings of the arrowheads, urn and wristguard C. Nicolas, Université Paris 1); $n^{\circ} 8$, Kermenhir barrow, Poullan-sur-Mer (after Chatellier, archives) ; $n^{\circ}$ 9, Kérimanton, Quéménéven, (after Briard, 1984a).

nord-sud et mesure $1,5 \mathrm{~m}$ de longueur et $1 \mathrm{~m}$ de largeur. Le mobilier funéraire se trouvait sous l'orthostate oriental qui était renversé. Il se compose d'un gobelet campaniforme décoré de lignes faites à la coquille de coque (Salanova, 1996), d'un vase plat sans ornement, d'une pierre à cupule, d'un brassard d'archer et d'une pendeloque en schiste, d'un bandeau, de trois agrafes et d'une spirale en or (Gaillard, 1884).

$N^{\circ} 14$ : Kerallant, Saint-Jean-Brévelay, Morbihan (fig. 16, $n^{\circ} 14$ )

Le «dolmen » de Kerallant, fouillé par Fernand de Cussé en 1886, a été compris dans un premier temps comme une allée couverte ruinée (L'Helgouach et al., 1970). En effet, l'un des orthostates de la tombe est orné d'une hache emmanchée en bas-relief, signe attribué au Néolithique moyen (Boujot et al., 2000 ; Sparfel et Pailler, 2009) ou final (L'Helgouach et al., 1970). Dans un deuxième temps, Jacques Briard (1989) a suggéré que la tombe de Kerallant correspondait à un coffre campaniforme. Cette deuxième interprétation a pu être confirmée grâce au plan inédit de la tombe conservé à la Société polymathique du Morbihan (Rapport, $n^{\circ}$ 590). La tombe de Kerallant est constituée de trois orthostates, d'un muret en pierres sèches et d'un pavage interne le long des murs. Orientée nord-sud, elle mesure 2,3 m de longueur pour 1,65 m de largeur. Le mobilier, conservé au musée de Vannes, comprend les restes de quatre gobelets campaniformes, un couteau-grattoir sur lame en silex du Grand-Pressigny (détermination N. Mallet), un brassard d'archer en schiste noir et une perle tubulaire en tôle d'or pour Kerallant (Cussé, 1886). Les quatre gobelets comprennent un vase non-décoré, conservé dans sa quasi-totalité, dix-huit tessons qui permettent de reconstituer imparfaitement le profil d'un gobelet décoré à la cordelette (type AOC), sept tessons (un bord et six fragments de panse et de carène) d'un gobelet de type maritime décoré probablement à la coque (Cerastoderma edule ; Salanova, 2000) et un fragment de panse, également de type maritime, qui s'en différencie par un décor imprimé par un coquillage plus fin. Deux hypothèses sont plausibles pour la construction du coffre de Kerallant :

- Les trois orthostates, dont la stèle ornée, correspondraient aux vestiges d'une sépulture mégalithique (tombe à couloir ou allée couverte) vidangée et fermée au Campaniforme par un muret en pierre sèches au sud.
- Le coffre aurait été construit, comme à La Guette $\left(\mathrm{n}^{\circ} 11\right)$ et à Roh $\mathrm{Du} \mathrm{B}\left(\mathrm{n}^{\circ} 12\right)$, avec une architecture combinant dalles de chant et murs en pierres sèches. La stèle ornée serait en réemploi, comme dans les cas des statues-menhirs féminines réutilisées sous forme de fragments dans le tumulus de Kerméné (Guidel, Morbihan ; Giot, 1960) ou en dalle de couverture dans le tumulus de l'Âge du Bronze ancien de Kersandy (Plouhinec, Finistère ; Briard et al., 1982).

\section{DES COFFRES MÉGALITHIQUES CAMPANIFORMES AUX TUMULUS ARMORICAINS}

$\mathrm{Au}$ premier regard, ces quatorze tombes campaniformes semblent assez diverses par leur architecture et leur mobilier. Néanmoins, la typochronologie des gobelets campaniformes et l'étude des mobiliers associés permettent de regrouper ces sépultures en trois étapes correspondant à celles récemment définies pour la façade atlantique (Salanova, 2011) (tabl. III).

Les sépultures campaniformes les plus anciennes (étape 1) sont celles de Kerallant et de La Guette. Dans les deux cas, on trouve de la céramique de type maritime ou de type AOC, types réputés les plus anciens (Lanting et Van der Waals, 1976 ; Lanting, 2008 ; Salanova, 2011 ; fig. 16, n 11 et 14). Les tombes de Kerallant et de la Guette, toutes deux orientées nord-sud, ont une architecture assez similaire, employant dalles de chant et murets en pierres sèches. Ces deux sépultures, situées à l'intérieur de la Bretagne, attestent la présence ancienne du Campaniforme dans cette zone (fig. 17).

La phase récente du Campaniforme (étape 2) est représentée par six tombes, plus ou moins bien documentées : Kerouaren, Roh Du B, Plobannalec-Lesconil 1, Kercadoret, le coffre P de Plobannalec-Lesconil et Kermenhir. Les décors des gobelets campaniformes sont constitués de motifs linéaires en bandes (Roh Du B), en panneau sur toute la panse (Kerouaren) ou sur le col (Kermenhir). Ces lignes sont réalisées par impression de coquille ou par incision. Ces décors se rangent dans le style épimaritime, variante régionale des céramiques aux bandes hachurées de type maritime (Salanova, 2011). Les vestiges du vase non-décoré du coffre P de Plobannalec-Lesconil illustrent une grande qualité de fabrication, similaire à celle du gobelet lisse de Kerallant (fig. 16, $\mathrm{n}^{\circ} 14$ ). À cette étape, apparaissent dans les sépultures individuelles les premières armes à base de cuivre (hache plate, poignard à soie) et les pointes de flèches en silex. Les tombes de Plobannalec-Lesconil 1 et de Kercadoret 


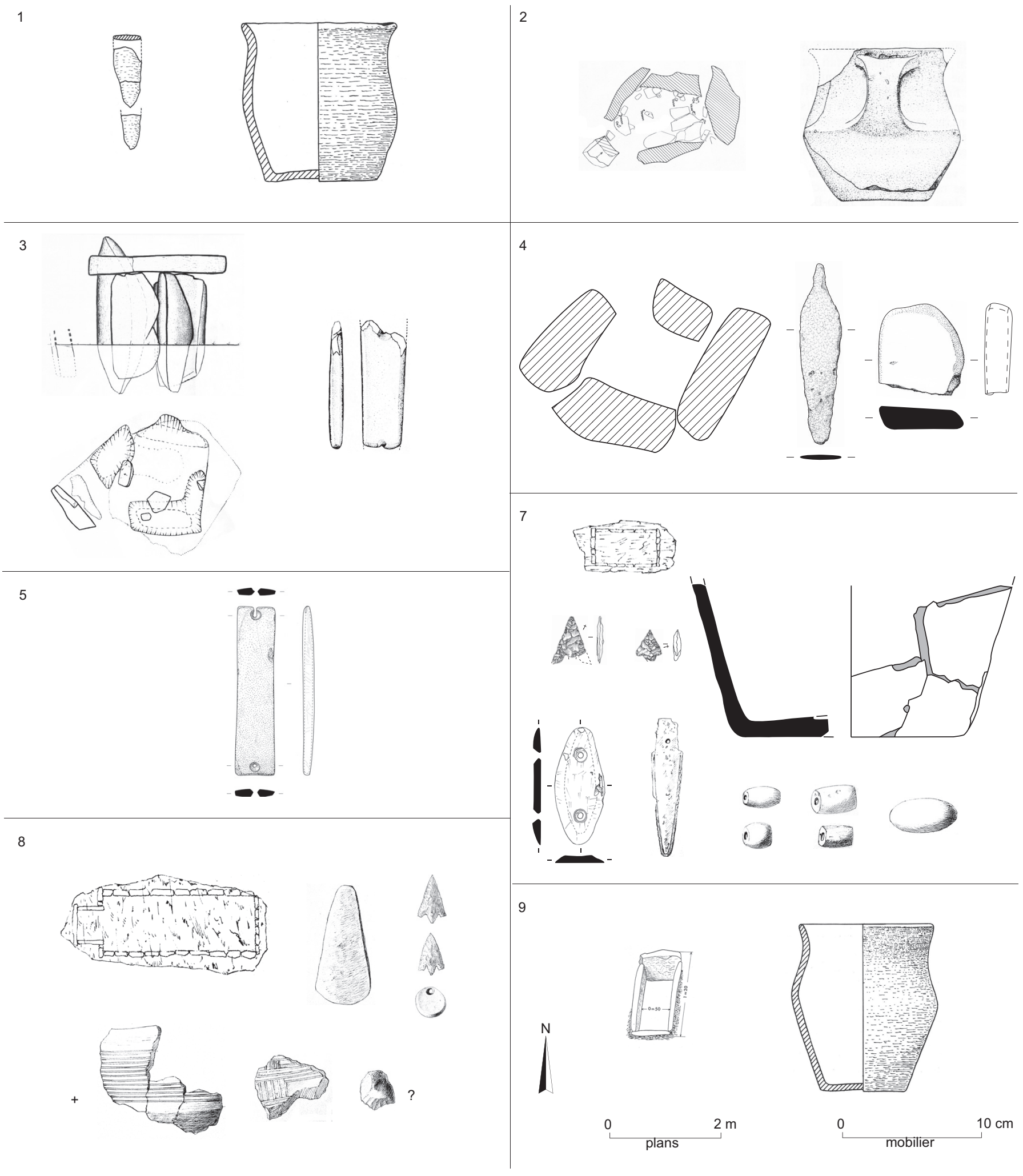

ont toutes deux livré un brassard d'archer à deux trous de forme allongée (fig. 16, $\mathrm{n}^{\circ} 3$ et 5 ). Ces deux brassards sont typologiquement proches de l'exemplaire de Kerouaren (fig. 16, $\mathrm{n}^{\circ} 13$ ), ce qui permet de les attribuer à l'étape 2. Les architectures funéraires gardent des liens avec l'étape ancienne (orientation nord-sud de la tombe de Kerouaren, coffre mixte de Roh Du B) mais elles se diversifient (coffre mégalithique, mixte ou en pierres sèches, recouvert ou non par un tumulus, orientation préférentiellement est-ouest).

Six tombes peuvent être rattachées à la fin du Campaniforme (étape 3) : Coatjou-Glas, Bignat, Lothéa, PlobannalecLesconil 2, Kerimanton, Juno-Bella. Les formes des vases, sans décors, s'éloignent du modèle de cloche renversée. La hauteur de la carène tend à remonter (Kérimanton, Bignat), le 
11

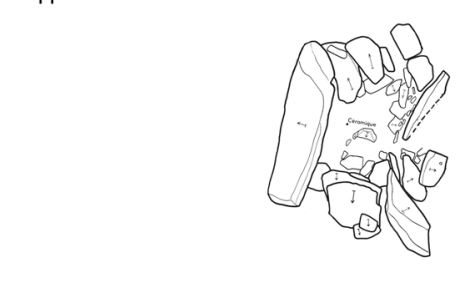

13
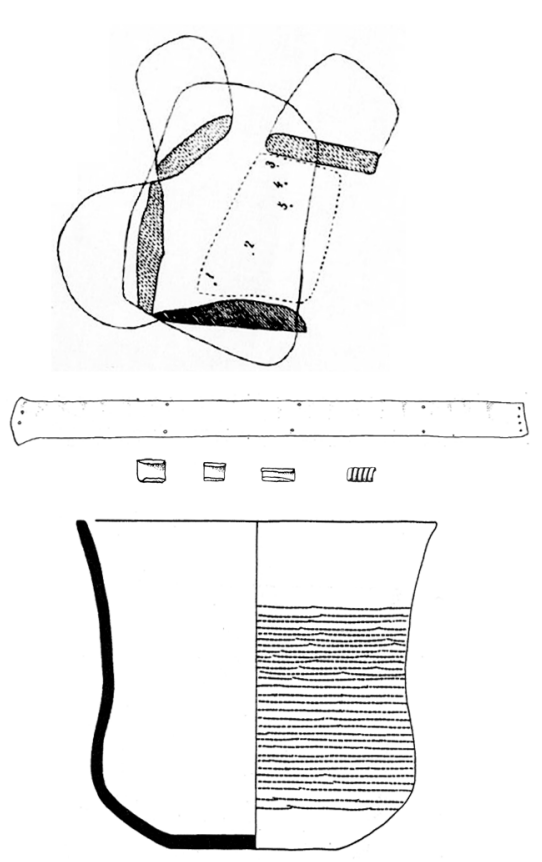

17

$\stackrel{N}{1}$
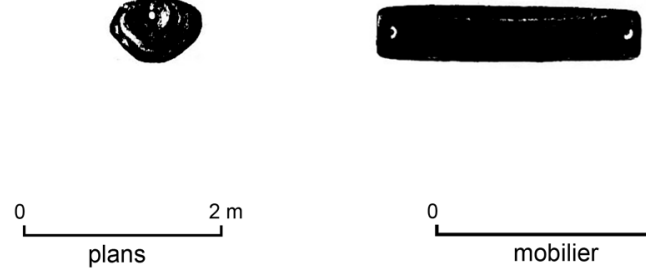

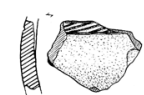

12
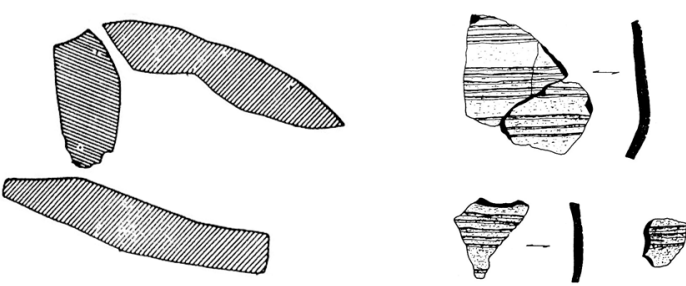

14
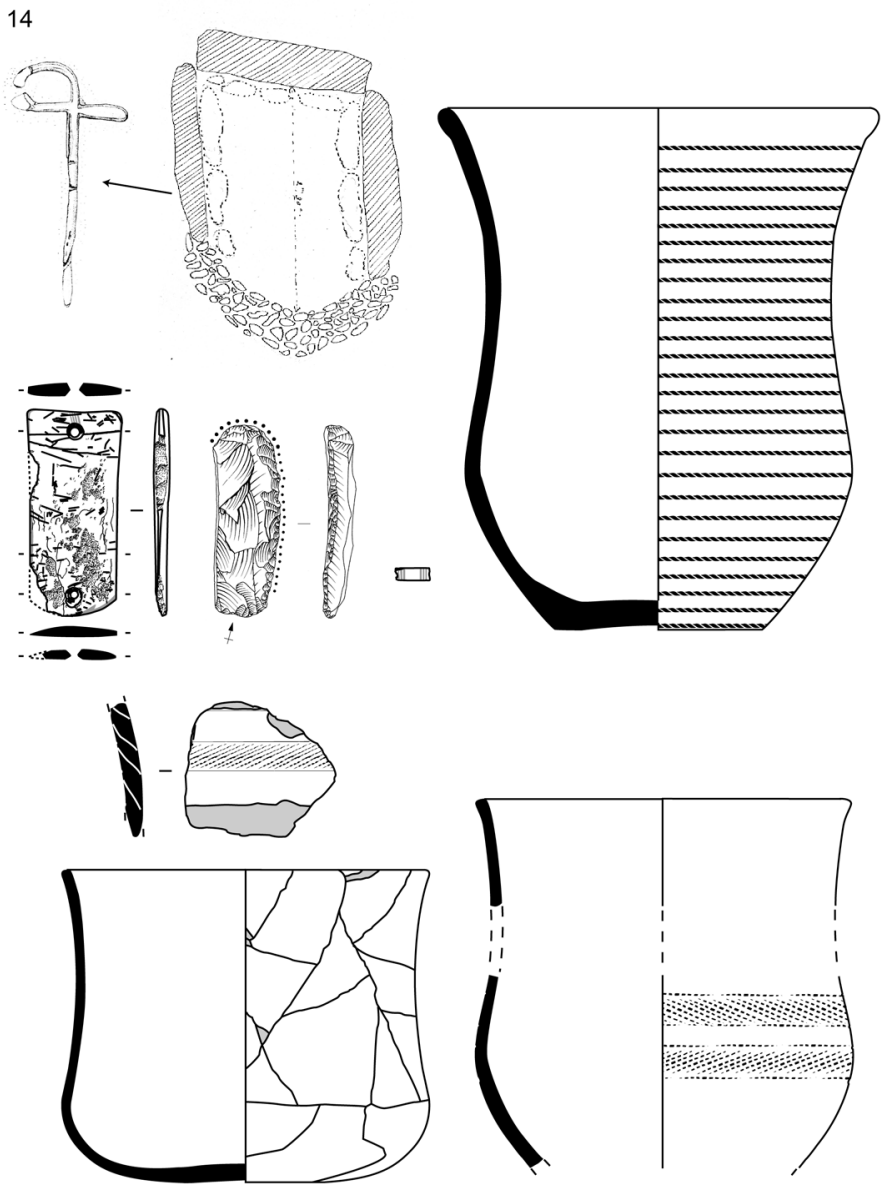

Fig. 16bis - Plan et mobiliers des sépultures individuelles campaniformes de Bretagne. lle-et-Vilaine : $n^{\circ} 11$, La Guette, Paimpont (d'après Briard, 1989); Morbihan : $n^{\circ} 12$, Roh-du B, La Chapelle-Neuve (d'après Briard, 1989) ; $n^{\circ} 13$, Kerouaren, Plouhinec (plan et dessin lithique d'après Gaillard, 1884, dessin métallique d'après Eluère, 1982, dessin céramique d'après Salanova, 2000) ; $n^{\circ} 14$, Kerallant, Saint-Jean-Brévelay (plan F. de Cussé d'après cliché C. Le Pennec, ( ) Archives de la Société polymathique du Morbihan, relevé de gravure d'après L'Helgouach et al., 1970, dessins mobilier: C. Nicolas, Université Paris 1).

Plans and grave goods of individual Bell Beakers graves from Brittany. Ile-et-Vilaine: $n^{\circ} 11$, La Guette, Paimpont (d'après Briard, 1989); Morbihan: $n^{\circ} 12$, Roh-du B, La Chapelle-Neuve (d'après Briard, 1989); $n^{\circ} 13$, Kerouaren, Plouhinec (plan and drawings of the lithics after Gaillard, 1884, drawings of the golden objects after Eluère, 1982, drawing of the Beaker after Salanova, 2000); $n^{\circ} 14, K^{2}$ Kallant, SaintJean-Brévelay (plan F. De Cussé after photograph C. Le Pennec, (C) Société polymathique du Morbihan Archives, drawing of the engraving after L'Helgouach et al., 1970, drawings of the grave goods C. Nicolas, Université Paris 1).

fond plat (Coatjou-Glas) et l'anse (Juno-Bella) font leur apparition (fig. $16, \mathrm{n}^{\text {os }} 1,3,7$ et 9). Ces changements sont également perceptibles au niveau des brassards d'archer : forme en losange arrondi (Coatjou-Glas), quatre perforations contre deux auparavant (Lothéa, Plobannalec-Lesconil 2). L'évolution la plus notable se fait au niveau de la métallurgie. Le trou de rivet et la décoration de filet parallèle au bord font leur apparition sur le poignard de Coatjou-Glas, puis la dimension des lames augmentent considérablement sur les exemplaires type Quimperlé de Lothéa (fig. 14 et $16, n^{\circ} 7$ ). On trouve toujours des parures en « schiste » (Chatellier, 1887) et des pointes de flèches en silex. Dans le cas de Lothéa, les éléments de parure en or, en argent 
Tabl. III - Typochronologie des sépultures individuelles campaniformes de Bretagne (en gris foncé, étape 1 ; en gris clair, étape 2 ; en blanc, étape 3).

Typochronology of the Bell Beakers individual graves from Brittany (dark gray, stage 1; Early Bell Beaker; light grey, stage 2; Bell Beaker; white, stage 3; Late Bell Beaker).

\begin{tabular}{|c|c|c|c|c|c|c|c|c|c|c|c|c|c|c|c|c|c|c|c|c|c|c|}
\hline \multirow[b]{2}{*}{ Tombes } & \multicolumn{15}{|c|}{ Mobilier sérié } & \multicolumn{6}{|c|}{ Architecture } & \multirow[b]{2}{*}{ Autre mobilier } \\
\hline & 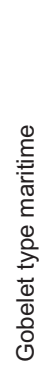 & 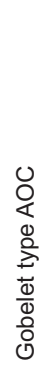 & 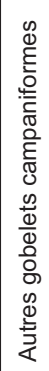 & 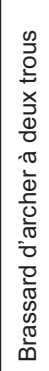 & 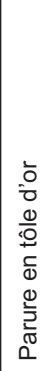 & 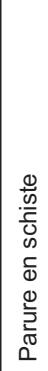 & 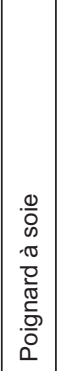 & 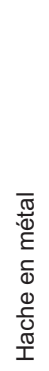 & 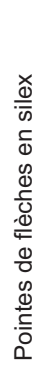 & 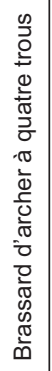 & 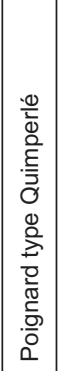 & 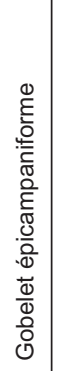 & 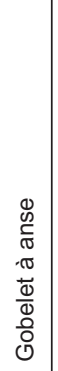 & 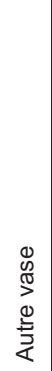 & 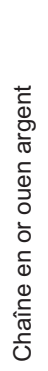 & 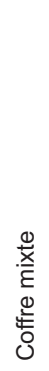 & 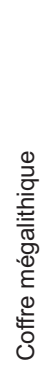 & 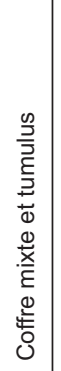 & 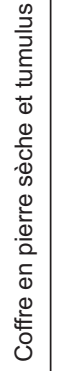 & 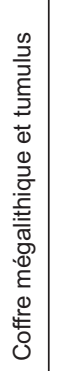 & 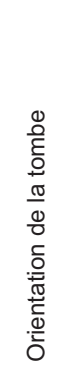 & \\
\hline La Guette, Paimpont & 0 & & & & & & & & & & & & & & & 0 & & & & & N-S & \\
\hline $\begin{array}{l}\text { Kerallant, Saint- } \\
\text { Jean-Brévelay }\end{array}$ & 0 & 0 & 0 & 0 & 0 & & & & & & & & & & & 0 & & & & & N-S & $\begin{array}{l}\text { un couteau-grattoir en } \\
\text { silex du Grand-Pressigny }\end{array}$ \\
\hline $\begin{array}{l}\text { Kerouaren, } \\
\text { Plouhinec }\end{array}$ & & & 0 & 0 & 0 & 0 & & & & & & & & & & & 0 & & & & N-S & une pierre à cupule \\
\hline $\begin{array}{l}\text { Roh du B, La } \\
\text { Chapelle-Neuve }\end{array}$ & & & 0 & & & & & & & & & & & 0 & & & & 0 & & & E-O & \\
\hline $\begin{array}{l}\text { Plobannalec- } \\
\text { Lesconil } 1\end{array}$ & & & & 0 & & & & & & & & & & & & & & & & & $?$ & \\
\hline $\begin{array}{l}\text { Kercadoret, Moëlan- } \\
\text { sur-Mer }\end{array}$ & & & & 0 & & & & & & & & & & & & & 0 & & & & - & \\
\hline $\begin{array}{l}\text { Coffre P, } \\
\text { Plobannalec- } \\
\text { Lesconil }\end{array}$ & & & $?$ & & & & 0 & & & & & & & & & & 0 & & & & E-O & $\begin{array}{l}\text { une pierre ponce et un } \\
\text { polissoir en grès }\end{array}$ \\
\hline $\begin{array}{l}\text { Kermenhir, Poullan- } \\
\text { sur-Mer }\end{array}$ & & & $?$ & & & 0 & & 0 & O & & & & & & & & & & O & & E-O & un grattoir en silex (?) \\
\hline $\begin{array}{l}\text { Coatjou-Glas, } \\
\text { Plonéis }\end{array}$ & & & & 0 & & 0 & 0 & & 0 & & & & & 0 & & & & & 0 & & E-O & \\
\hline Bignat, Kérien & & & & & & & ? & & & & & 0 & & & & & & & ? & & ? & \\
\hline Lothéa, Quimperlé & & & & & & & & 0 & 0 & 0 & 0 & & & & 0 & & & & & 0 & E-O & $\begin{array}{l}\text { une baguette «en cuivre», } \\
\text { une pendeloque en } \\
\text { jadéitite, un galet de } \\
\text { quartzite }\end{array}$ \\
\hline $\begin{array}{l}\text { Plobannalec- } \\
\text { Lesconil } 2\end{array}$ & & & & & & & & & & 0 & & & & & & & & & & & ? & \\
\hline $\begin{array}{l}\text { Kerimanton, } \\
\text { Quéménéven }\end{array}$ & & & & & & & & & & & & 0 & & & & & 0 & & & & $\begin{array}{l}\text { SE- } \\
\text { NO }\end{array}$ & \\
\hline Juno-Bella, Berrien & & & & & & & & & & & & & 0 & & & & 0 & & & & E-O & \\
\hline
\end{tabular}

et en jadéitite restent une exception (fig. 12 et $15, \mathrm{n}^{\circ} 5$ ). Les modes architecturaux de l'étape 3 sont variés et en continuité avec l'étape précédente.

Ces trois étapes de sépultures individuelles attestent l'existence en Bretagne de ce mode d'inhumation et de leur évolution (mobilier, architecture, orientation) tout au long de la période campaniforme. Du point de vue de la chronologie absolue, ces tombes individuelles ne sont pas d'un grand secours. Les fouilles anciennes, les contextes remaniés et l'absence de vestiges organiques laissent peu d'espoir à de futures datations au radiocarbone. C'est donc hors de la Bretagne qu'il faut aller chercher des éléments de datation. De cette manière, Laure Salanova (2011) a récemment proposé de situer la phase ancienne (étape 1) du Campaniforme breton entre 2500 et 2400 avant notre ère, la phase récente (étape 2) entre 2400 et 2150 avant notre ère et la phase finale (étape 3) entre 2150 et 1950 avant notre ère (fig. 18).
Cette dernière étape du Campaniforme breton serait contemporaine des premiers tumulus armoricains de l'Âge du Bronze ancien (Needham, 2000a ; Nicolas et al., à paraître).

Les coffres mégalithiques ou mixtes des étapes 1 et 2 permettent de discerner un horizon clairement campaniforme à l'origine du millier de tombes (Briard, 1984), sous tumulus ou non, de l'Âge du Bronze ancien en Bretagne (fig. 17). La nature mégalithique de certaines architectures funéraires n'est pas exclusive au Campaniforme et de grandes pierres continuent à être employées à l'Âge du Bronze ancien, fréquemment pour la couverture mais également pour les parois (Briard, 1984). L'aspect nettement mégalithique de certains caveaux campaniformes illustre la réinvention à cette période d'une architecture individuelle funéraire, cette réinvention se faisant parfois aux dépens de sépultures collectives plus anciennes (tombe de Kerallant et coffre $\mathrm{P}$ de Plobannalec-Lesconil). 


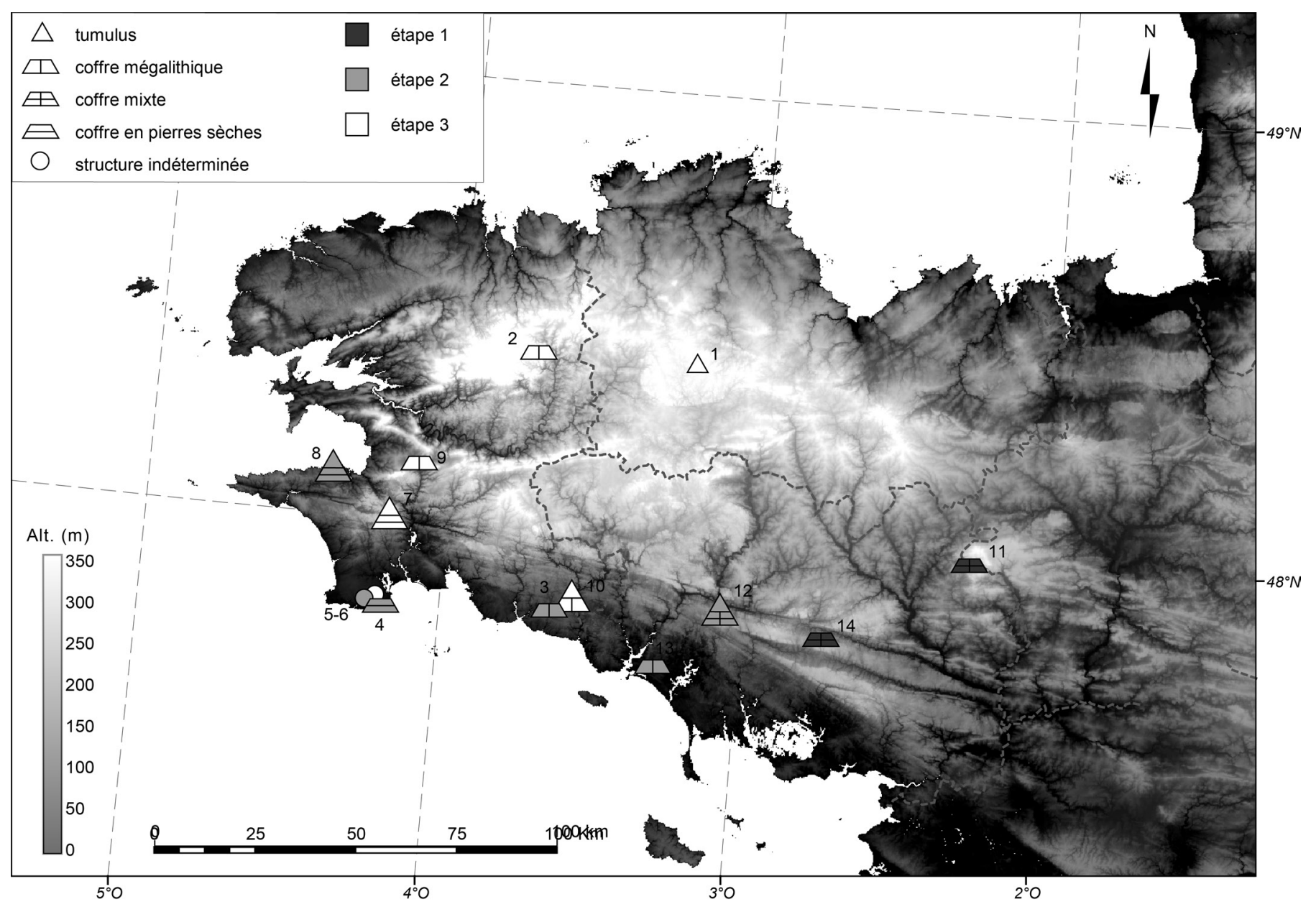

Fig. 17 - Carte des sépultures individuelles campaniformes de Bretagne (Carte : P. Stephan, CNRS). Côtes-d'Armor: $n^{\circ} 1$, Bignat, Kérien ; Finistère : $n^{\circ} 2$, Juno-Bella, Berrien; $n^{\circ} 3$, Kercadoret, Moëlan-sur-Mer ; $n^{\circ} 4$, Coffre $P$, nécropole de Lesconil, PlobannalecLesconil ; nos 5-6, Plobannalec-Lesconil ; $n^{\circ} 7$, Coatjou-Glas, Plonéis ; $n^{\circ}$ 8, Kermenhir, Poullan-sur-Mer ; $n^{\circ} 9$, Kerimanton, Quéménéven; $n^{\circ} 10$, Lothéa, Quimperlé ; Ille-et-Vilaine : $n^{\circ} 11$, La Guette, Paimpont ; Morbihan : $n^{\circ} 12$, Roh Du B, La ChapelleNeuve ; $n^{\circ} 13$, Kerouaren, Plouhinec ; $n^{\circ} 14$, Kerallant, Saint-Jean-Brévelay.

Map of the individual Bell Beaker graves from Brittany (Map: P. Stephan, CNRS). Côtes-d'Armor: $n^{\circ} 1$, Bignat, Kérien ; Finistère: $n^{\circ} 2$, Juno-Bella, Berrien; $n^{\circ}$ 3, Kercadoret, Moëlan-sur-Mer; $n^{\circ}$ 4, Cist P, nécropole de Lesconil, Plobannalec-Lesconil; $n^{o s}$ 5-6, PlobannalecLesconil; $n^{\circ} 7$, Coatjou-Glas, Plonéis; $n^{\circ} 8$, Kermenhir, Poullan-sur-Mer; $n^{\circ}$ 9, Kerimanton, Quéménéven; $n^{\circ}$ 10, Lothéa, Quimperlé; IIle-et-Vilaine: $n^{\circ} 11$, La Guette, Paimpont; Morbihan: $n^{\circ} 12$, Roh Du B, La Chapelle-Neuve; $n^{\circ} 13$ Kerouaren, Plouhinec $n^{\circ}$ 14, Kerallant, Saint-Jean-Brévelay.

Parallèlement, on voit se développer des enveloppes tumulaires de taille toujours croissante, du cairn de $3 \mathrm{~m}$ de diamètre de $\mathrm{La}$ Guette au tertre de $24 \mathrm{~m}$ de diamètre de Kermenhir. L'imposant tumulus de Lothéa avec sa tombe, qualifiée anciennement et à juste titre de « dolmen » (Chatellier, 1851, p. 58), constitue un jalon supplémentaire entre l'architecture mégalithique du Néolithique et l'émergence d'une monumentalité renouvelée : la tombe individuelle sous tumulus circulaire.

\section{LOTHÉA ET LA FAÇADE ATLANTIQUE}

Les rapprochements les plus frappants ont été faits entre les chaînes en or et en argent de Lothéa et des exemplaires provenant du Portugal et d'Espagne (Hernando Gonzalo, 1983 ; Briard, 1984 ; Eluère, 1986). Les chaînes de spirales d'or ou d'argent ont été trouvées en assez grand nombre dans la péninsule Ibérique, mais malheureusement elles proviennent de contextes très mal datés, parfois de dépôts (Soeiro, 1982 ; Delibes de Castro, Del Val Recio, 2005). Leur attribution chronologique varie du Bronze ancien au Bronze final suivant les auteurs. Leur datation au Bronze ancien repose essentiellement sur la trouvaille de Lothéa (Hernando Gonzalo, 1983, p. 106-110). Il n'est pas assuré que toutes ces chaînes soient contemporaines. Néanmoins la similitude est telle entre les chaînes de Lothéa et certains exemplaires ibériques que ceux-ci sont probablement contemporains. On retrouve les mêmes caractères dans l'exemplaire en argent de Antas de Ulla (Pontevedra, Galice, Espagne ; 


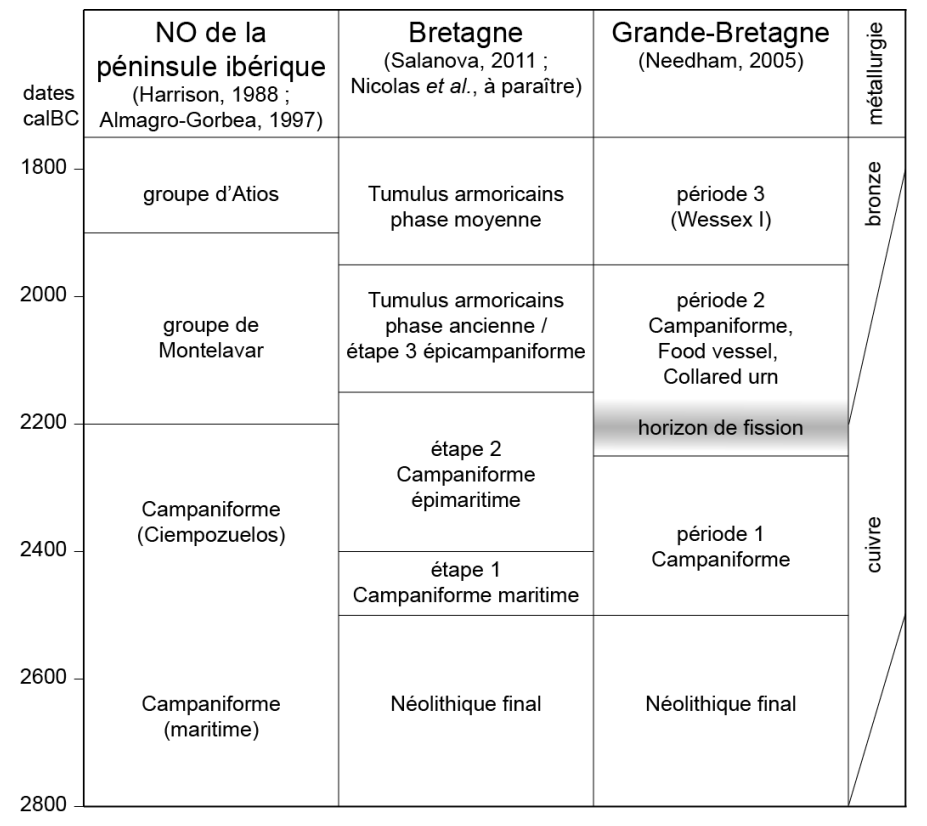

Fig. 18 - La fin du $3^{\mathrm{e}}$ millénaire avant notre ère le long de l'Europe atlantique.

The end of the Third millennium BC along the Atlantic Europe.

Hernando Gonzalo, 1983) : combinaison d'un grand anneau et d'anneaux plus petits, chaînes d'anneaux décroissants, spirales aux extrémités appointées (fig. 19, n 1). On peut citer un couple de spirales en argent découvert dans un recoin de la grotte de La Vaquera (Terreiglesias, Segovia, Espagne) ou la chaîne de spirales en or de Sequeade (Barcelos, Portugal) dont la facture rappelle les spirales de Lothéa, à la seule différence que leur section est lenticulaire (Soeiro, 1982 ; Delibes de Castro, Del Val Recio, 2005). Un élément vient confirmer la distribution atlantique de ces spirales en France. Il s'agit d'" une douzaine de morceaux de fil de métal jaune d'un millimètre et demi environ, grossièrement arrondis, roulés en spirale et formant un collier » découverte à Singleyrac (Dordogne) avec un squelette, une hache plate en bronze, un poignard rhodanien de $40 \mathrm{~cm}$ de long et un tesson orné d'une ligne en chevrons (Labet, 1859, p. 81). On peut signaler la découverte à Beuzec-Conq (Concarneau, Finistère), d'une sépulture dont le mobilier rappelle celui de Lothéa. Cette tombe a livré, sans plus de précision, « une spirale d'or, une grande hache plate en bronze, deux pointes de flèches à ailerons en silex et une pendeloque en pierre polie » (Halna du Fretay, 1889, p. 358). Une des deux flèches a pu être dessinée par P. du Chatellier (1879) ; elle est de type Kerguévarec, un des plus anciens types de flèche armoricaine (Nicolas, 2011).

Les trouvailles d'objets en or sont relativement limitées dans les tombes de l'Âge du Bronze ancien en Bretagne, où l'or est essentiellement utilisé pour réaliser des clous minuscules qui viennent décorer le manche ou des pastilles qui ornent la lame des poignards en bronze (Briard, 1984). En revanche, les éléments de parure en or, rattachables à la culture campaniforme, sont nombreux et variés (Le Rouzic, 1930 ; Eluère, 1982 ; Salanova, 2000). Ceux-ci présentent des analogies frappantes entre Bretagne et péninsule Ibérique : colliers à lamelles découpées ou gargantillas de tiras, appliques discoïdes et bandeaux. D'autres éléments de nature plus ubiquiste (spirale, perle tubulaire, agrafe) sont également connus. Ces parures réalisées en tôle d'or montrent par leur facture et leurs décors une grande unité autour du Golfe de Gascogne (Eluère, 1982, p. 222), suggérant l'échange d'objets ou la circulation d'artisans. Leur distribution reste fondamentalement atlantique, malgré des inventaires complets pour la France et la péninsule Ibérique (Eluère, 1982 ; Hernando Gonzalo, 1983 ; Ambruster, Parreira, 1993 ; Ladra et al., 2003). Trois concentrations de ces parures peuvent être observées : autour de l'estuaire du Tage, en Galice et sur la côte atlantique française, de l'estuaire de la Gironde au golfe du Morbihan. Étonnamment, les chaînes à spirales ont exactement la même distribution et participent selon toute vraisemblance du même réseau de circulation (fig. 20). Ces trois concentrations de parure en or ont des échos dans la répartition d'autres mobiliers. Estuaire du Tage, Galice et Golfe du Morbihan sont trois régions intensément marquées par la culture campaniforme, avec une nette prédominance de gobelets de type maritime à décors de bandes hachurées. La similitude des formes et des techniques décoratives des vases implique d'ailleurs le déplacement de personnes et des transferts techniques (Salanova, 2000 ; Prieto Martinez, Salanova, 2009). Les pointes de Palmela en cuivre, autre objet symbole de la diffusion du Campaniforme, se trouvent en grand nombre dans la péninsule Ibérique. Leur répartition en France suit deux voies : la Méditerranée et les côtes atlantiques, où une concentration s'observe entre l'estuaire de la Gironde et le golfe du Morbihan (Briard, Roussot-Laroque, 2002). Ces relations atlantiques au Campaniforme semblent se dérouler à partir du milieu du $3^{\mathrm{e}}$ millénaire avant notre ère (Bailly, Salanova, 1999; Lanting, 2008 ; Salanova, 2011).

Les poignards de Lothéa ont été comparés avec des grandes lames en cuivre arsénié en Europe continentale, en GrandeBretagne et dans la péninsule ibérique. Cependant seuls les exemplaires ibériques offrent de véritables parallèles par leurs formes et leurs décors (Briard, Mohen, 1974 ; Delibes et al., 1982 ; Gomez de Soto, 1990 ; Brandherm, 2003). Il s'agit notamment de huit poignards regroupés sous le type Quinta da Romeira par Dick Brandherm (2003, p. 136-138). Le type Quinta da Romeira se retrouve de la Galice au sud-est de l'Espagne. Cependant seules cinq lames mesurant plus de $30 \mathrm{~cm}$ proviennent du Portugal ou de Galice. Sur ces cinq lames, on retrouve la même sinuosité des tranchants avec une garde nettement évasée et une soie resserrée identique aux armes de Lothéa (fig. 19, $\mathrm{n}^{\text {os }} 3$ à 6). Les poignards peuvent être décorés de un à deux filets parallèles aux bords. Seul l'emmanchement est différent. Le manche présente, comme à Lothéa, une lunule centrale, mais sa fixation à la lame ne se fait pas par des rivets. De légers rebords au niveau de la soie des poignards de type Quinta da Romeira servent sans doute à fixer le manche. Trois de ces cinq poignards révèlent une composition de cuivre arsénié (Krause, 2003 ; Brandherm, 2003, p. 136-138 ; Tabl. II). Sur la lame de la tombe de Quinta de Agua Branca (Vila Nova de Cerveira, Minho, Portugal) on retrouve le même éclat argenté donné par l'arsenic (Ambruster, Parreira, 1993, p. 37).

Ces grands poignards à soie développée résultent selon toute vraisemblance d'une évolution des poignards campaniformes et d'un savoir-faire métallurgique grandissant, à 

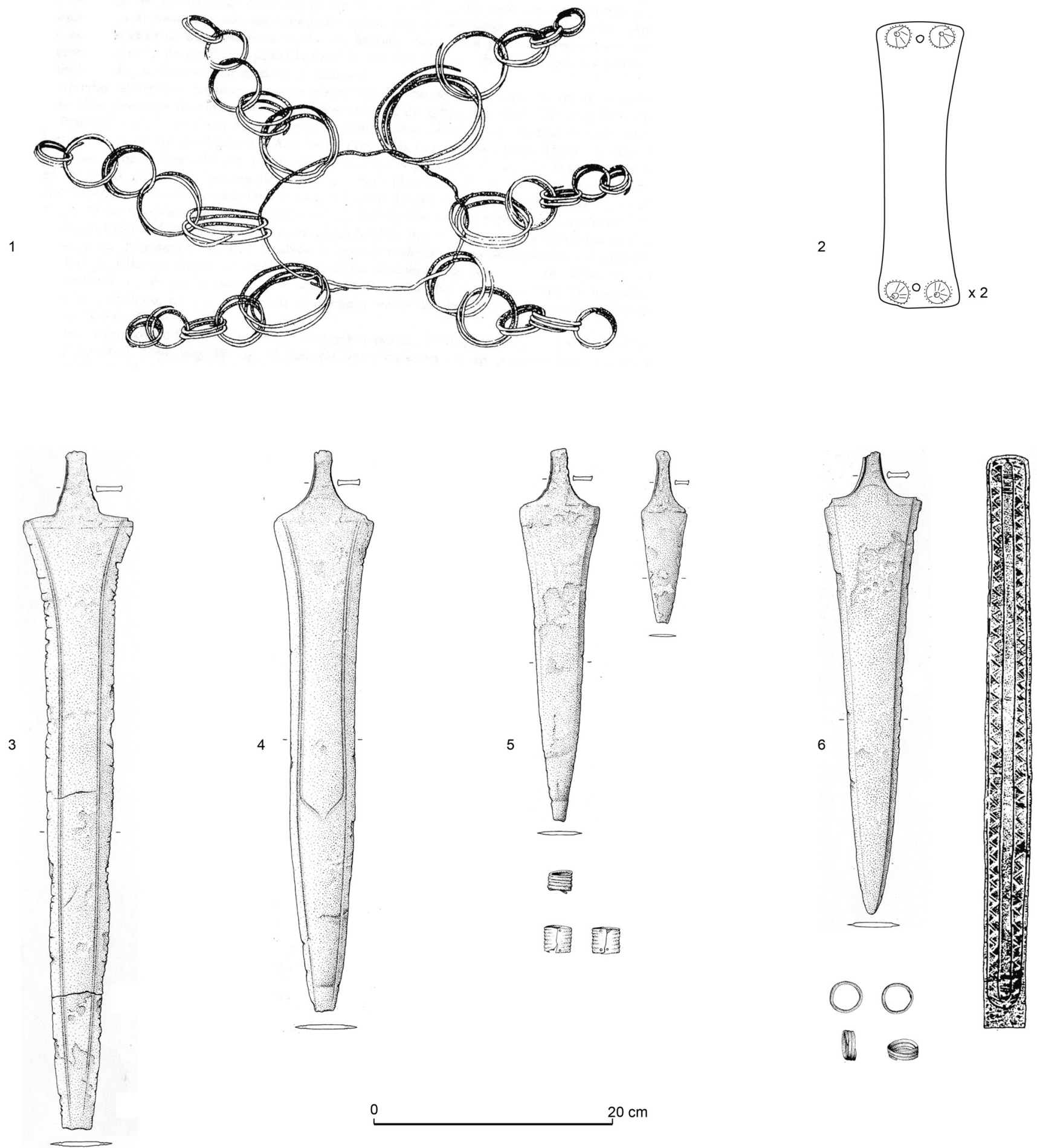

même de réaliser permettant la réalisation des grandes lames dépassant les $50 \mathrm{~cm}$ (Delibes et al., 1982 ; Guilaine, Briois, 1984 ; Gomez de Soto, 1990). D'autres grands poignards sont connus en France et en Galice, mais ils diffèrent des types Quimperlé et Quinta da Romeira par leur soie triangulaire (Briard, 1965 ; Guilaine, Briois, 1984 ; Brandherm, 2003) ou peu marquée (Almagro Gorbea, 1972; Delibes et al., 1982 ; Gomez de Soto, 1990). Leur composition métallique révèle également des cuivres arséniés et participe d'une même ambiance atlantique. La comparaison faite avec la grande lame en cuivre arsénié de Gau-Bickelheim (Rhénanie-Palatinat, Allemagne) (Hundt, 1971) semble peu pertinente, puisque celle-ci présente un emmanchement et des décors pour partie inexistants en Bretagne. Elle illustre au mieux un influx du monde atlantique vers l'est (Gomez de Soto, 1990, p. 222). Un seul poignard de type Quimperlé est connu en Grande- 
Fig. 19 - Sélection d'armes et de parures en métal de Galice et du nord du Portugal. N 1, chaîne en argent de Antas de Ulla, Pontevedra, Galice ; $n^{\circ} 2$, brassard d'archer en tôle d'or de Vila Nova de Cerveira, Minho, Portugal ; $n^{\circ} 3$, poignard en cuivre arsénié de Pinhal dos Melos, Fornos de Algofres, Beira Alta, Portugal ; $n^{\circ} 4$, poignard de Porto Mouro, Boiro, La Corogne, Galice ; $n^{\circ} 5$, poignards, spirale d'argent et deux cylindres en or de la tombe en ciste d'Atios, Porriño, Pontevedra, Galice ; $n^{\circ} 6$, poignard en cuivre arsénié, bandeau, deux anneaux et deux spirales en or de Quinta da Agua Branca, Vila Nova de Cerveira, Minho, Portugal (poignards d'après Brandherm, 2003, brassard d'après Ambruster, Parreira, 1993, parures d'après Hernando Gonzalo, 1983).

Selection of weapons and metal ornaments from Galicia and northern Portugal. $N^{\circ} 1$, silver chain from Antas de Ulla, Pontevedra, Galicia ; $n^{\circ} 2$, gold sheet wristguard from Vila Nova de Cerveira, Minho, Portugal; $n^{\circ} 3$, arsenical copper dagger from Pinhal dos Melos, Fornos de Algofres, Beira Alta, Portugal ; $n^{\circ} 4$, dagger from Porto Mouro, Boiro, A Coruña, Galicia ; $n^{\circ} 5$, daggers, silver spirals and two golden cylinders from the Atios cist, Porriño, Pontevedra, Galicia ; $n^{\circ}$ 6, arsenical copper dagger, golden band, two rings and two spirals from Quinta da Agua Branca, Vila Nova de Cerveira, Minho, Portugal (daggers after Brandherm, 2003, wristguard after Ambruster, Parreira, 1993, ornaments after Hernando Gonzalo, 1983).

Bretagne, parmi de nombreux poignards de type armoricobritannique (Piggot, 1938 ; Gerloff, 1975). Il s'agit vraisemblablement d'un poignard importé, trouvé dans un dépôt avec deux bracelets larges en or et les fragments de deux gobelets à Lockington (Leicestershire ; Needham, 2000b).

Un dernier élément peut nous tourner vers la péninsule Ibérique : le brassard d'archer. Il reste difficile de faire des comparaisons pour ce type d'objet, dont les variantes sont peu nombreuses. Cependant quelques tendances se dégagent à l'échelle de l'Europe. Le brassard de Lothéa correspond au type 3 d'Edward Sangmeister et il en est l'unique représentant en France. Ce type est attesté dans le domaine alpin et dans la péninsule Ibérique (Sangmeister, 1964). Le brassard en tôle d'or de Vila Nova de Cerveira (Viana do Castelo, Portugal), imitant les perforations biconiques des exemplaires en pierre, est un bon exemple de comparaison (fig. 19, $\mathrm{n}^{\circ} 2$ ).

\section{LOTHÉA ET LA FIN DU III ${ }^{e}$ MILLÉNAIRE AVANT NOTRE ÈRE}

Armes et parures de Lothéa offrent des connexions fortes avec la péninsule Ibérique. D'un point de vue culturel ces objets sont à relier au Campaniforme mais apparaissent plutôt évolués. Ils s'inscrivent dans les nombreux changements techniques et culturels qui s'opèrent à la fin du $\mathrm{III}^{\mathrm{e}}$ millénaire avant notre ère, le long de la façade atlantique de l'Europe. Le passage de la métallurgie du cuivre à celle du bronze s'opère de façon discontinue entre 2200 et 1800 avant notre ère (Needham, 2005 ; Strahm, 2007). Dans le même temps, la culture campaniforme, uniforme à ses débuts, se diversifie peu à peu jusqu'à créer une mosaïque culturelle au début de l'Âge du Bronze ancien. C'est ce qu'a démontré Stuart Needham (2005) pour la GrandeBretagne avec ses processus de fusion et de fission. La coexistence en Bretagne de tombes épicampaniformes et de tumulus armoricains s'inscrit dans cette logique. Dans le nord-ouest ibérique, on observe un abandon progressif des éléments de la culture matérielle campaniforme. Les groupes de Montelavar et d'Atios rassemblent des sépultures individuelles au mobilier riche (Harrison, 1974 ; Almagro Gorbea, 1997 ; Brandherm, 2007), parmi lesquelles les tombes d'Atios et de Quinta de Agua Branca (fig. 19, $\mathrm{n}^{\text {os }} 5$ et 6). Ces deux groupes montrent l'abandon du gobelet campaniforme, puis des pointes de
Palmela et des brassards d'archer au profit de nouveaux styles céramiques et de nouveaux poignards (dont le type Quinta da Romeira).

Ces évolutions dans la culture matérielle restent mal situées dans la chronologie absolue. Ce problème est, entre autres, dû à la courbe de calibration des dates radiocarbone, « le millénaire des dates comprises entre 4000 et $3000 \mathrm{BP}$ ayant duré en réalité plus de 1200 ans » (Voruz, 1996, p. 107). Cette imprécision a contribué à proposer une date haute, vers 2300 avant notre ère, pour le début de l'Âge du Bronze ancien (Voruz, 1996). Dans le même temps, trop peu de datations absolues fiables ont été effectuées sur les tombes de Bretagne (Nicolas et al., à paraître). Martín AlmagroGorbea (1997) s'est servi des tumulus armoricains et de la tombe de Lothéa pour caler chronologiquement les groupes de Montelavar et d'Atios, ce qui nous semble un raisonnement un peu spécieux dans la mesure où les tumulus armoricains sont très mal datés. Par comparaison avec les sites du reste de l'Espagne datés au radiocarbone, Richard Harrison (1988) a proposé une période allant de 2200 à 1900 avant notre ère pour le groupe de Montelavar (fig. 18). Seule la Grande-Bretagne offre pour l'heure un corpus de sites et de dates radiocarbones suffisamment important pour élaborer une périodisation de la fin du $3^{\mathrm{e}}$ millénaire avant notre ère. (Needham, 2005 ; Sheridan, 2007 ; fig. 18). Ainsi, Stuart Needham (2005) a situé l'« horizon de fission », qui voit une diversification culturelle et des innovations métallurgiques, entre 2250 et 2150 avant notre ère. En Bretagne, le tumulus de Brun Bras (Saint-Adrien, Côtesd'Armor) fournit un terminus ante quem pour Lothéa. Au regard de la typochronologie des flèches armoricaines (Nicolas, 2011), les armatures de Brun Bras (types Kerguévarec et Kernonen) apparaissent comme plus récentes que celles de Lothéa. Le cercueil en bois de Brun Bras a été daté à $3650 \pm 35$ BP (GrN-7176), soit 2137-1929 calBC à $2 \sigma$ (Briard, 1984). En Galice, le squelette de la tombe de Quinta de Agua Branca (fig. 19, n 6) donne une date plus récente : $3570 \pm 50 \mathrm{BP}$ (Beta-230330), soit 2109-1755 calBC à $2 \sigma$ (Bettencourt, 2010). Deux datations radiocarbone ont été réalisées sur le fourreau du poignard type Quimperlé de Lockington donnent des dates contradictoires s'étendant sur la deuxième moitié du 3e millénaire et au-delà : $3910 \pm 60$ BP (OxA-6173) et $3630 \pm 55 \mathrm{BP}(\mathrm{OxA}-6447)$, donnant respectivement en dates calibrées à $2 \sigma$ 2568-2206 calBC et 2195-1786 calBC (Hughes, 2000). Ces datations permettent au mieux d'attribuer le tumulus de Lothéa à la fin du $3^{\mathrm{e}}$ millénaire avant notre ère. Compte-tenu de la précocité des tumulus armoricains, nous serions enclins à le situer vers le XXII" siècle avant notre ère. 


\section{CONCLUSION}

Le mobilier de Lothéa s'inscrit dans de larges connexions le long de l'Europe atlantique, sur lesquelles s'accordent de nombreux auteurs. Ces connexions sont plus ou moins resserrées et s'expriment ici ou là par des convergences de formes, des échanges d'idées, des circulations d'objets, des transferts de technologie ou des déplacements d'individus. Malgré cette relative homogénéité, des différences sont perceptibles. Les relations sont multiples entre Bretagne et péninsule Ibérique au Campaniforme. Elles semblent s'estomper assez rapidement à l'Âge du Bronze ancien au profit des îles Britanniques et de l'Irlande. La tombe de Lothéa apparaît comme une des dernières expressions de ces échanges. Par la suite, aucun objet ne semble provenir d'Espagne ou du Portugal avant l'Âge du Bronze final (Briard, 1998). En revanche, les relations avec le nord de l'Europe atlantique s'intensifient avec l'arrivée de parures en ambre, en jais ou en faïence, transitant par la Manche. Dans le même temps, poignards en métal et vases de Bretagne se retrouvent en Angleterre (Needham, 2000a ; Tomalin, 1988). Ce moment correspond à la culture du Wessex, qui, à partir des débuts du $\mathrm{II}^{\mathrm{e}}$ millénaire avant notre ère, se distingue entre autres par l'accumulation de biens exotiques.

La tombe de Lothéa est manifestement l'un des plus anciens tumulus armoricains (Briard, 1984). Culturellement une partie du mobilier de Lothéa évoque ce qui se passe en Espagne. Les chaînes en or et en argent de Lothéa proviennent vraisemblablement de la péninsule Ibérique. Les grands poignards trouvés de part et d'autre du golfe de Gascogne sont de toute évidence le fruit d'un même savoir-faire, partagé par des artisans travaillant le cuivre arsénié et capables de produire des armes d'une longueur inégalée en Europe. À partir de là, il est permis de s'interroger sur l'origine et le statut du défunt de Lothéa : s'agit-il d'un personnage venu de la péninsule Ibérique ou d'un chef de Bretagne ? Les objets exotiques de Lothéa sont-ils venus avec le défunt ou par colportage ? Ou bien ont-ils été réalisés sur place par des artisans ayant parcouru les mers?

Les voyages à longue distance d'individus, pressentis depuis longtemps dans la culture matérielle campaniforme, sont de mieux en mieux identifiés. En France, la sépulture d'Arenberg (Wallers, Nord) a livré deux vases de style typiquement britannique (Salanova et al., 2011). En Écosse, plusieurs tombes, dont celle d'Upper Largie Quarry (Kilmartin Glen, Argyll \& Bute), ont été édifiées suivant des règles connues aux Pays-Bas et renfermaient des vases de style hollandais (Cook et al., 2010, p. 198). Les analyses isotopiques du Strontium, substance qui se fixe dans les dents durant l'adolescence, révèlent des migrations insoupçonnées. Ainsi, la plus riche tombe campaniforme connue découverte à Amesbury près de Stonehenge (Wiltshire, Grande-Bretagne) a révélé les restes d'un homme qui a grandi dans une région située entre les Alpes et la Scandinavie (Chenery, Evans, 2011). Le statut de ces grands voyageurs n'est pas encore parfaitement établi. D'un côté, on peut voir dans la richesse des tombes des personnages de haut rang, accumulant les biens les plus rares en voyageant ou en entretenant des réseaux à longue distance (Needham, Woodward, 2008). De l'autre côté, la présence d'outils de métallurgistes dans certaines tombes pourrait suggérer l'existence d'artisans forgeant le métal et colportant l'innovation. Par leur maîtrise $\mathrm{du}$ feu et d'un savoir-faire ésotérique, ceux-ci finiraient par subjuguer les sociétés qu'ils rencontrent (Fitzpatrick, 2009).

De nombreuses questions restent en suspens mais la redécouverte de la relation de fouilles d'A. Boutarel aura permis de rappeler l'importance historique de la tombe de Lothéa. Cette sépulture devait renfermer les restes d'un grand personnage, héritier de la culture campaniforme et fondateur des élites qui régneront par la suite en Bretagne. Les « Princes d'Armorique » (Briard, 1984) continueront durant plusieurs générations à se faire enterrer avec poignards et haches en métal, armatures de prestige et parures exotiques.

\section{REMERCIEMENTS}

Cette étude n'aurait pu voir le jour sans la collaboration de nombreuses personnes et institutions. Nous tenons tout d'abord à remercier Alain Villes (conservateur, musée d'Archéologie nationale, Saint-Germain-en-Laye) et Isabelle Bardiès-Fronty (conservateur en chef, musée national du Moyen-Âge des thermes et de l'hôtel de Cluny, Paris) pour nous avoir permis d'étudier pleinement les objets de Lothéa. Nous remercions le laboratoire d'Archéosciences (UMR6566/CreAAH) pour nous avoir autorisé à utiliser leurs archives dans la présente publication, ainsi que Christophe Le Pennec (Musée d'Histoire et d'Archéologie de Vannes) pour nous avoir transmis le plan de la tombe de Kerallant. Sur le terrain, Yohann Sparfel, Yves Guéguen et Tangi Kervern nous ont prêté main forte. Louis Chauris, Pierre Pétrequin et Nicole Mallet nous ont aidés dans les déterminations pétrographiques. Yohann Sparfel nous a généreusement communiqué de nombreux documents sur les mégalithes et les tumulus de la région de Quimperlé. Enfin, Mike Ilett a aimablement corrigé le résumé en anglais. 


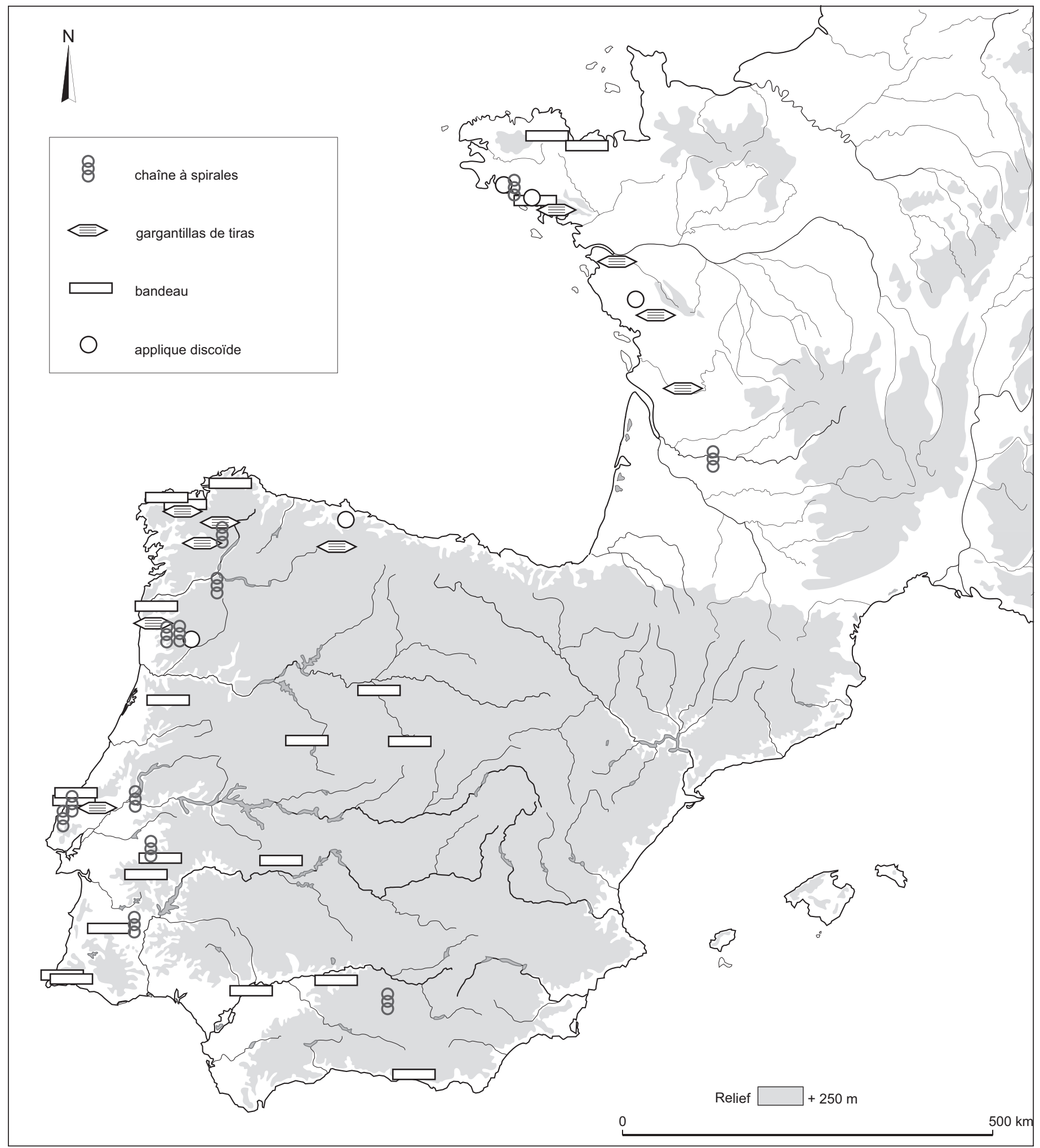

Fig. 20 - Répartition de parures en or campaniforme (d'après inventaires de Eluère, 1982, Hernando Gonzalo, 1983, Ambruster, Parreira, 1993, Ladra et al., 2003).

Distribution of Bell Beaker golden ornaments (after inventories of Eluère, 1982, Hernando Gonzalo, 1983, Ambruster, Parreira, 1993, Ladra et al., 2003). 


\section{BibLIOGRAPHIE}

\begin{tabular}{ll}
\multicolumn{1}{c}{ ABRÉVIATIONS } \\
AEIN & Association pour les Études interrégionales sur le Néolithique \\
APA & Association France Archéologie \\
APC & Association des Presses chauvinoises \\
BAAB & Bulletin archéologique de l'Association bretonne \\
BAR & British archaeological Report \\
BSAF & Bulletin de la Société archéologique du Finistère \\
BSHAPL & Bulletin de la Société d'Histoire et d'Archéologie du Pays de Lorient \\
BSPF & Bulletin de la Société préhistorique française \\
BSECN & Bulletin de la Société d'Émulation des Côtes-du-Nord \\
BSPM & Bulletin de la Société polymathique du Morbihan \\
BAPRAB & Bulletin de l'Association pour la Promotion des Recherches sur l'Âge du Bronze \\
RAASBLZB & Recueil des Actes de l'Académie des Sciences, Belles-lettres et Arts de Bordeaux \\
BSANDLI & Bulletin de la Société archéologique de Nantes et du Département de la Loire-inférieure \\
JBAA & Journal of the british archaeological Association \\
MSH & Maison des Sciences de l'Homme \\
PSO & Préhistoire du Sud-Ouest \\
SRA & Service régionale de l'Archéologie \\
REAP & Revue de l'École d'Anthropologie de Paris \\
RAO & Revue archéologique de l'Ouest \\
SPF & Société préhistorique française
\end{tabular}

Almagro Gorbea M.

1972: « La espada de Guadalajara y sus paralelos peninsulares ", Trabajos de Prehistoria, 29, p. 55-82.

1997: « La Edad del Bronce en la Península Ibérica : periodización y cronología », Saguntum, 30, p. 217-229.

Ambruster B., Parreira R. (Dir.)

1993: Inventário do museu nacional de arqueologia, Colecção de ourivesaria, 1. ${ }^{\circ}$ volume, Do Calcolitico à Idade do Bronze, Lisboa, Instituto Português de Museus, $239 \mathrm{p}$.

AnNic J.

2000: « Notices d'archéologie fini stérienne (années 1999-2000) : Quimperlé, forêt de Carnoët, BSAF, 129, p. 40-42.

Audran J.

1874: « La rive droite de la Laïta (excursion archéologique de Quimperlé au Pouldu) », BSAF, 1, p. 63-71.

Aveneau de la Grancière P.

1898: « Le Bronze dans le centre de la Bretagne-Armorique : fouille du tumulus à enceinte semi-circulaire de Saint-Fiacre en Melrand, canton de Baud », BSPM, p. 81-95.

1899: « Le Bronze en Bretagne-Armorique : de quelques sépultures de l'époque du Bronze en Armorique Occidentale», $B A A B$, XVII, p. 202-217.

BAILloud G.

1964 : Le Néolithique dans le Bassin parisien,
Paris, CNRS Editions (Suppl. à Gallia Préhistoire, 2), $394 \mathrm{p}$.

Bailly M., Salanova L.

1999: " Les dates radiocarbones du Campaniforme en Europe occidentale : analyse critique des principales séries de dates ", in Evin J., Oberlin C., Daugas J.-P., Salles J.-F. (DIR.), $14 \mathrm{C}$ et Archéologie, $3^{e}$ Congrès International, Paris, SPF (Coll. Mémoire de la SPF, 36), p. 219-224.

Balquet A.

2001: Les tumulus armoricains du Bronze ancien, Patrimoine archéologique de Bretagne, Rennes, Institut culturel de Bretagne et Association des travaux du Laboratoire d'anthropologie et de préhistoire de l'université de Rennes 1 (Coll. Patrimoine archéologique de Bretagne), $150 \mathrm{p}$.

\section{Bénard C., dit Le Pontois}

1929: Le Finistère préhistorique, Paris, Éd. Nourry, Publications de l'Institut international d'anthropologie, 3, $337 \mathrm{p}$.

BERCÉ F.

1979: Les premiers travaux de la Commission des monuments historiques : 1837-1848: procès-verbaux et relevés d'architectes, Paris, $\mathrm{A}$. et J. Picard, 452 p.

Bertrand A.

1884: La Gaule avant les Gaulois, d'après les monuments et les textes, Paris,
Ernest Leroux, 204 p.

1891: Nos origines, la Gaule avant les Gaulois, d'après les monuments et les textes, Paris, éd. Ernest Leroux, 349 p.

Bettencourt A. M. S.

2010: « La Edad del Bronce en el Noroeste de la Península Ibérica : un análisis a partir de las prácticas funerarias », Trabajos de Preistoria, 67, 1, p. 139-173.

Bonnardin S.

2009: La parure funéraire au Néolithique ancien dans les Bassins parisien et rhénan : Rubané, Hinkelstein, Villeneuve-Saint-Germain, Paris, SPF, (Coll. Mémoire de la SPF, 49), 322 p.

Bordreuil M., Bordreuil M.C., Jallot L. Remicourt M.

2008: " La "hachette-pendeloque": révision de l'inventaire pour la France Méditerranéenne ", in BROCHIER J. É., Guilcher A., Pagni M. (DIR.), Archéologies de Provence et d'ailleurs. Mélanges offerts à Gaëtan Congès et Gérard Sauzade, Aix-en-Provence, Association Provence Archéologie (Suppl. au Bulletin Archéologique de Provence, 5), p. 205-214.

Boujot C., Cassen S.

1992: « Le développement des premières architectures funéraires monumentales en France occidentale », in LE Roux C.-T. (DIR.), Paysans et bâtisseurs : l'émergence $d u$ Néolithique atlan- 
tique et les origines du mégalithisme, actes $d u 17^{e}$ Colloque interrégional sur le Néolithique, Vannes, 29-31 octobre 1990, Rennes, RAO (Suppl. à la Revue archéologique de l'Ouest, 5), p. 195-211.

Boujot C., Cassen S., Defaix J.

2000: «La pierre décorée du caveau et les gravures régionales nouvellement découvertes ", in CASSEN S. (DIR.), Éléments d'architecture. Exploration d'un tertre funéraire à Lannec er Gadouer (Erdeven, Morbihan). Constructions et reconstructions dans le Néolithique morbihannais. Propositions pour une lecture symbolique, Chauvigny, APC (Coll. Mémoires, XIX), p. 277-297.

BRANDHERM D.

2003: Die Dolche und Stabdolche der Steinkupfer-und der älteren Bronzezeit auf der Iberischen Halbinsel, Stuttgar, Franz Steiner Verlag (Coll. Prähistorische Bronzefunde, VI, 12), $540 \mathrm{p}$.

2007: «Algunas reflexiones sobre el bronce inicial en el noroeste peninsular. La cuestión del llamado horizonte "Montelavar" ", Cuadernos de Prehistoria y Arqueología, 33, p. 69-90.

BRIARD J.

1965: Les dépôts bretons et l'Age du Bronze atlantique, Rennes, Faculté des sciences (Coll. Travaux du Laboratoire d'anthropologie préhistorique), $352 \mathrm{p}$.

1970a: "Un tumulus du Bronze ancien : Kernonen en Plouvorn (Finistère) 》, L'Anthropologie, 74, p. 5-56.

1970b: " Les tumulus de l'Âge du Bronze de Plouvorn-Plouzévédé (Finistère) », $B S P F, 67$, p. 372-385.

1972: « Les tumulus de Kervini en Poullan fouilles de $1971 », B S A F, 98$, p. 21-38.

1978: « tumulus des monts d'Arrée, JunoBella à Berrien », BSAF, 106, p. 17-35.

1983: « La céramique des tumulus de l'âge du bronze du Morbihan », BSPM, 110, p. 93-110.

1984: Les Tumulus d'Armorique, Paris, Picard (Coll. " L'Âge du Bronze en France », 3), $304 \mathrm{p}$.

1998: «Flux et Reflux du Bronze Atlantique vus d'Armorique. Le Bronze Ancien », in Jorge S. O. (ÉD.), Existe uma idade di Bronze Atlântico ?, Lisboa, Instituto Português de Museus (Suppl. à Trabalhos de Arqueologia, 10), p. 114-123.

2000: "Jean L'Helgouach (1933-2000) », $B S P F, 97$, p. 332-333.

BRIARD J. (DIR.)

1989: Mégalithes de haute Bretagne, les monuments de la forêt de Brocéliande et du Ploërmelais : structures, mobilier et environnement, Paris, Éditions de la
MSH (Coll. Documents d'Archéologie française, 23), $135 \mathrm{p}$.

Briard J., Bourhis J., Le Provost F., ONNÉE Y.

1977a: «Un tumulus du Bronze ancien avec maison funéraire à Saint-Jude, Bourbriac, Côtes du Nord », BSPF, 74, p. $622-641$

Briard J., Bourhis J.-J., Van Schoor M.

1998: « La paléométallurgie campaniforme du Portugal. Nouvelles données sur ses compositions métalliques et ses relations atlantiques ", $B S P F, 95$, p. 393-402.

Briard J., Le Goffic M., Onnée Y.

1982: «Les fouilles de Kersandy à Plouhinec (Finistère) : une tombe du Bronze Ancien à "déesse-mère" néolithique ", $B S A F, 110$, p. 17-39.

Briard J., Le Goffic, M., Onnée Y.

1994: Les tumulus de l'Âge du Bronze des Monts d'Arrée, Rennes, Institut culturel de Bretagne (Coll. Patrimoine archéologique de Bretagne), 96 p.

Biard J., L'Helgouach J.

1957: Chalcolithique, Néolithique secondaire, survivance néolithique à l'Age du Bronze Ancien en Armorique, Rennes, Faculté des Sciences (Coll. Travaux du Laboratoire d'Anthropologie préhistorique), $72 \mathrm{p}$

Briard J., Mohen J.-P.

1974: « Le tumulus de la forêt de Carnoët à Quimperlé (Finistère) », Antiquités nationales, 6, p. 46-60

Briard J., Onnée Y., Veillard J.-Y.

1977b:L'âgeduBronzeauMuséedeBretagne, Rennes, Musée de Bretagne, 170 p.

Briard J., Roussot-Laroque J.

2002: «Les débuts de la métallurgie dans la France atlantique ", in Bartelheim M., Pernicka E., Krause R. (éd.), Die Anfänge des Metallurgie in der alten Welt / The Beginnings of Metallurgy in the Old World, Rahden/Wetsf, Verlag Marie Leidorf $\mathrm{GmbH}$ (Coll. Forschungen zur Archäometrie und Altertumswissenschaft), p. 135-160.

Brunet P., André M.-F., Bemilli C. Brunet V., Cottiaux R., Durand J., Gosselin R., Le Jeune Y., Renard C.

2004: « Deux sites de la fin du Néolithique en vallée de la Marne : Lesches "le Pré du Refuge" et Meaux "Route de Varreddes" (Seine-et-Marne), résultats préliminaires ", in Journée d'information du 20 novembre 2004, Paris, Internéo, $n^{\circ} 5$, Saint-Germain-en-Laye, AEIN, p. 101-113.

Butler J.-J., Waterbolk H.

1974: « La fouille de A.-E. Van Giffen à la Motta, un tumulus de l'Âge du Bronze ancien à Lannion (Bretagne) », Palaeohistoria, 16, p. 107-167.

Cambry J.

1799: Voyage dans le Finistère ou état de ce département en 1794 et 1795, Paris, Imprimerie-librairie du Cercle social, 2 vol., 598 p.

Chaigneau C.

2009: « Avec les antiquaires, les monuments mégalithiques passent du statut d'objets littéraires à celui d'objets d'étude scientifique », in Sparfel Y. et Pailler Y. (DIR.), Les mégalithes de l'arrondissement de Brest, Saint-Malo et Rennes, Centre régional d'Archéologie d'Alet et Institut culturel de Bretagne (Coll. Patrimoine archéologique de Bretagne), p. 15-17.

Chatellier A. (DU)

1844: " tumulus de la forêt de Carnoët », Bulletin monumental, 10, p. 229-230.

1851: « Lecture d'une notice adressée au Congrès par M. Duchatellier », provèsverbal de séance, $B A A B, 3$, p. 52-59.

Chatellier P. (du)

Archives: Collection Paul du Chatellier, série 100J, Quimper, Archives départementales du du tumulus \& du menhir du Run, en Tréffiagat (Finistère) », $B S E C N, 16$, p. 1-7.

1881: «Dolmens et chambres sépulcrales de Kervinion, Kervadel et Kerfuns en Plobannalec (Finistère) », Matériaux pour l'Histoire primitive et naturelle de l'Homme, $2^{\mathrm{e}}$ s., 12 , p. 265-275.

1883a: «Exploration de quelques sépultures de l'époque du Bronze dans l'ouest et le sud du département du Finistère », $B S E C N, 21$, p. 1-37.

1883b: « Nouvelles explorations dans les communes de Plozévet et de Plouhinec (Finistère), sépultures de l'époque du Bronze ", Revue archéologique, $3^{\mathrm{e}}$ s., 2, p. 1-19.

1887: « Sépulture de Coatjou-Glas en Plonéis (Finistère) », Matériaux pour l'Histoire primitive et naturelle de l'Homme, $3^{\mathrm{e}} \mathrm{s}$., 4, p. $49-52$

1889: Les époques préhistoriques et gauloises dans le Finistère, Inventaire des monuments de ce département des temps préhistoriques à la fin de l'occupation romaine, Paris, éd. E. Lechevalier, 211 p.

1897: " Exploration sur les montagnes d'Arrhées et leurs ramifications, années 1895-1896 », BSECN, 25, p. 51-112.

1907: Les époques préhistoriques et gauloises dans le Finistère, Inventaire des monuments de ce département des temps préhistoriques à la fin de l'occupation romaine, $2^{\mathrm{e}}$ édition, RennesQuimper, éd. Plihon \& Hommay, 391 p.

Chenery C. A., Evans J. A.

2011: «A Summary of the Strontium 
and Oxygen Isotope Evidence for the Origins of Bell Beaker individuals found near Stonehenge ", in FitzPatrick A. (Éd.), The Amesbury Archer and the Boscombe Bowmen : Bell Beaker burials at Boscombe Down, Amesbury, Wiltshire, Salisbury, Wessex Archaeology (Coll. Wessex Archaeology Report, 27), p. 185-190.

Cogné J., Horrenberger J.-C., Morzadec M.-T.

1973: Notice explicative, feuille de Lorient, Orléans, Bureau de Recherches géologiques et minières (Coll. Carte géologique de la France à 1/50 000, VII-20), $19 \mathrm{p}$.

Cook M., Ellis C., Sheridan A. (avec les contributions de Barber J., Bonsall C., Bush H., Clarke C., Crone A., Engl R., Fouracre L., Heron C., JaY M., McGibbon F., Macsween A., Montgomery J., Pellegrini M., Sands R., SAVIlle A., ScotT D., Šoberl L., VANDORPe P.)

2010 : « Excavations at Upper Largie Quarry, Argyll \& Bute, Scotland : New Light on the Prehistoric Ritual Landscape of the Kilmartin Glen », Proceedings of the prehistoric Society, 76, p. 165-212.

Cussé F. (DE)

1886: «Fouille de deux dolmens en SaintJean-Brévelay», BSPM, p. 70-72.

Delibes G., Avello J. L., Rojo M. A.

1982: "Espadas del Bronce Antiguo y Medio halladas en la provincia de León », Zephyrus, 34-35, p. 153-163.

Delibes de Castro G., Del Val Recio J. M.

2005: «Espiraliformes de plata de la cueva de la Vaquera (Segovia) : un probable conjunto votivo de los inicios de la Edad del Bronce ", Munibe, 57, p. 301-313.

Donnart K.

2011: « Le matériel de mouture de l'habitat campaniforme / Bronze ancien de Beg ar Loued (île Molène, Finistère) : étude préliminaire ", in Buchsenschutz O., JACCOTtey L., JodRy F., Blanchard J.-L. (Dir.), Evolution typologique et technique des meules du Néolithique à l'an mille, actes des III Rencontres archéologiques de l'archéosite gaulois, à Saint-Julien sur Garonne, du 2 au 4 octobre 2009, Bordeaux, éd. de la Fédération Aquitania (Suppl. à Aquitania, 23), p. 435-445.

Eluère C.

1982: Les ors préhistoriques, Paris, Picard (Coll. L'Âge du Bronze en France), $287 \mathrm{p}$.

1986: «L'orfèvrerie pré-romaine au musée de Cluny », La Revue du Louvre et des Musées de France, 36, 2, p. 97-106.
EUZENot (ABBÉ)

1868: "Note sur une fouille faite au dolmen de Lez-Variel, en Guidel, le 4 novembre », $B S P M$, p. 176-178.

1878: « Guidel et ses antiquités », $B A A B, 21$, p. 193-230.

Fitzpatrick A.

2009: "In his hands and in his head: the Amesbury Archer as magician », in Clark P. (Éd.), Bronze Age Connections : Cultural Contact in Prehistoric Europe, Oxford, Oxbow Books, p. 176-188.

Fokkens H., Achterkamp Y., KuiJpers M. 2008: «Bracers or Bracelets ? About the Functionality and Meaning of Bell Beaker Wrist-guards », Proceedings of the prehistoric Society, 74, p. 109-140.

FonTÈs F.

1881: «Fouille à Carnac. Tombe circulaire », BSPM, p. 121-123.

Fréminville C.-P. De la Poix, dit Chevalier (De)

1832: Antiquités de la Bretagne : Le Finistère, Brest, éd. Le Fournier et Deperiers.

1845: Le guide du voyageur dans le département $d u$ Finistère, $2^{\mathrm{e}}$ édition, Brest, Imprimerie de Ch. Le Blois.

Gaillard F.

1884: Une série d'explorations à Plouhinec: le tumulus du Gruigen, les dolmens de Kerouaren, de Beg-en-Hâvre et du Mané-Bras, Vannes, Imp. Galles, 13 p.

1906: «L'atelier de silex taillés et de pierre polie du rocher de Beg-er-Goalennec en Quiberon (Morbihan) », L'Homme préhistorique, 4, p. 289-301.

Gallay G.

1981: Die kupfer- und altbronzezeitlichen: Dolche und Stabdolche in Franckreich, München, Beck (Coll. Prähistorische Bronzefunde, VI, 5), 164 p.

Galliou P.

2009: «Le Passé recomposé. Les réutilisations de monuments du Néolithique et de l'âge du Bronze en Armorique à l'âge du Fer et à l'époque romaine ", Les Cahiers de l'Iroise, 208, p. 97-132.

Galliou P. (avec la participation de Philippe E.)

2010: Le Finistère, Paris, Académie des Inscriptions et Belles-Lettres (Coll. Carte archéologique de la Gaule, 29), $495 \mathrm{p}$.

GANDOIS H.

2008: L'apparition du bronze à l'étain dans le Nord-ouest de la France, mémoire de master 1, université de Paris 1, 140 p.

2011: " L'adoption du bronze à l'étain en France », BAPRAB, 8, p. 73-74.
GerLOFF S.

1975: The Early Bronze Age daggers in Great Britain and a reconsideration of the Wessex culture, München, C.H. Beck'sche Verl (Coll. Prähistorische Bronzefunde, VI, 2), 298 p.

Giot P.-R .

1953: "Quelques tombes finistériennes de l'Âge du Bronze découvertes fortuitemen », BSAF, 79, p. 31-42.

1960: «Une statue-menhir en Bretagne (ou le mystère archéologique de la femme coupée en morceaux ", BSPF, 57, p. $317-330$

1990: "Chronique de préhistoire et de protohistoire finistérienne et des archéosciences pour 1990 », BSAF 119, p. 11-39.

1997: « Paul du Chatellier, sa famille, sa vie, son œuvre, sa collection ", Antiquités nationales, 29, p. 35-44.

Giot P.-R., Briard J., Pape L.

1979: Protohistoire de la Bretagne, Rennes, éd. Ouest-France, 443 p.

Giot P.-R., Cogné J.

1955: « Étude pétrographique des haches polies de Bretagne, IV.- Les haches de combat en métahornblendite ", $B S P F$, 52, p. 401-409.

GoMez dE Sото J.

1990: «Intégration atlantique et exotisme au Bronze ancien : le cas du glaive de Cissac en Médoc (Gironde) ", in L'Helgouach J. (DIR.), La Bretagne et l'Europe préhistoriques : mémoire en hommage à Pierre-Roland Giot, Rennes, Laboratoire d'Archéométrie, Université de Rennes 1 (Suppl. à la Revue archéologique de l'Ouest, 2), p. 221-225.

GouÉZIN P.

2007: Les mégalithes du Morbihan littoral (au sud des Landes de Lanvaux, de Guidel à Quiberon), Rennes-SaintMalo, Institut culturel de BretagneCentre régional d'Archéologie d'Alet (Coll. Les Dossiers du Centre régional d'archéologie d'Alet), $135 \mathrm{p}$.

Guigues J.

1970: «Les minéraux alluvionnaires de Basse-Bretagne ", Penn ar Bed, 60, p. $239-248$.

Guilaine J., Briois F.

1984: « L'épée de Lafage (Saint-Amadou, Ariège) », $B S P F, 81$, p. 122-125.

Halna du Fretay M. C. M.

1889: «Les âges préhistoriques et le début de l'ère chrétienne : Incinérations et inhumations dans le Finistère et l'Ouest de la Bretagne », BSAF, 16, p. 319-368.

Harrison R. J.

1974: «A Closed Find from Cañada Rosal, 
Prov. Sevilla, and Two Bell Beakers " Madrider Mitteilungen, 15, p. 77-94.

1988: « Bell Beakers in Spain and Portugal: working with radiocarbon dates in the 3rd millennium BC », Antiquity, 62, p. 464-472.

Henry Y.

2008: Les dépôts de collections archéologiques du Musée de Cluny au Musée de Saint-Germain-en-Laye, mémoire de master I, École du Louvre, Paris, 62 p.

Hernando Gonzalo A.

1983: « La orfebreria durante el Calcolitico y el Bronce antiguo en la peninsula iberica ", Trabajos de Prehistoria, 40, p. $85-138$

Hughes G.

2000: The Lockington Gold Hoard : an Early Bronze Age Barrow Cemetery at Lockington, Leicestershire, Oxford, Oxbow Books, 116 p.

Hundt H.-J.

1971 : «Der Dolchhort von Gau-Bickelheim in Rheinhessen ", Jahrbuch des RömischGermanischen Zentralmuseums Mainz, 18 , p. $1-43$.

Jeudy F., Maitre A., Praud I., Pétrequin A.M., Pétrequin P.

1997: « Les lames de pierre polie de Chalain 3 », in Pétrequin P. (ÉD.), Les sites littoraux néolithiques de Clairvauxles-Lacs et de Chalain (Jura), III, Chalain station 3. 3200-2900 av. J.-C, Paris, éd. de la MSH, vol. 2, p. 455-465.

Krause R.

2003: Studien zur kupfer- und frühbronzezeitlichen Metallurgie zwischen Karpatenbecken und Ostsee, Rahden, Leidorf (Coll. Vorgeschichtliche Forschungen), 338 p., 1 Cd-Rom.

LABET J.-A.

1859: « Découverte d'une sépulture gauloise aux environs de Bergerac ", RAASBLZB, 21, p. 81-83.

Ladra L., Silva A. C. F., Sousa M. J.

2003: " Notas sobre uma nova lâmina áurea de tiras do Norte do Portugal », Portugalia, 24, p. 53-64.

LANTING J. N.

2008: « De NO-Nederlandse/NW-Duitse Klokbekergroep : Culturele achtergrond, typologie van het aardewerk, datering, verspreiding en graftritueel », Palaeohistoria, 50, p. 11-326.

Lanting J. N., VAN der WaAls J. D.

1976: " Beaker culture relations in the Lower Rhine basin ", in LANTING J. N., VAN DER WAALS J. D. (DIR.), Glockenbecher Symposion, Oberried 1974, Haarlem, Fibula-van Dishoeck, p. 1-80.
L'Helgovach J.

1965: Les sépultures mégalithiques en Armorique (dolmens à couloir et allées couvertes), Rennes, Faculté des Sciences (Coll. Travaux du Laboratoire d'Anthropologie préhistorique), $330 \mathrm{p}$.

1970: « Le monument mégalithique du Goërem à Gâvres (Morbihan) », Gallia Préhistoire, 13, p. 217-261.

1995: « Le groupe armoricain à vases campaniformes ", in Giot P.-R., BRIARD J., PAPE L., La Protohistoire de la Bretagne, Rennes, éd. Ouest-France, p. 39-51.

L'Helgouach J., Bellancourt G., Gallais C., Lecornec J.

1970: « Sculptures et gravures nouvellement découvertes sur des mégalithes de l'Armorique ", $B S P F, 67$, p. 513-521.

LE Bihan J.-P.

1993: Un habitat de transition NéolithiqueAge duBronze, Quimper, Penancreac'h, D.F.S., SRA Bretagne, Rennes, 107 p.

Le Bihan J.-P, Robic J.-T., Tinevez J.-Y.

1994: « Notice d'archéologie finistérienne (année 1993) : Quimper, Penancreac'h », BSAF, 122, p. 111-115.

Le Bihan J.-P., Villard J.-F.

2010: Archéologie d'une île à la pointe de l'Europe: Ouessant. Tome 2, l'habitat de Mez Notariou, des origines à l'âge $d u$ Bronze, Saint-Thonan, Centre de Recherches archéologiques du Finistère-Cloitre imprimeur, $588 \mathrm{p}$.

Le Carguet H.

1911: " Les coffrets de pierre et les squelettes de Feunteunigou en Plouhinec ", $B S A F, 38$, p. 334-349.

Le Goffic M.

1996: « Notices d'archéologie finistérienne (année 1995) : Guengat, Kervouster », $B S A F, 125$, p. 45-46.

1999: « Notices d'archéologie finistérienne (année 1998) : Guengat, Kervouster », 128, p. 45-48.

Le Goffic M., Peuziat J.

1991: "Fouilles de sépultures en coffre de l'âge du bronze en Primelin, Plouhinec et Sizun-Saint-Cadou ", $B S A F, 120$, p. 33-54.

Le Men R.-F.

1868: «Fouilles d'un tumulus dans la forêt de Carnoët, commune de Quimperlé (Finistère) ", Revue archéologique, 17, p. 364-368.

1877 : «Statistique monumentale du Finistère (époque celtique) », $B S A F, 4$, p. 85-135.

Le Pontois L.

1890: " Le tumulus de Cruguel en Guidel », Revue archéologique, 16, p. 304-338.

Le Provost F., Giot P.-R., Onnée Y.

1972: «Prospections sur les collines de Saint-Nicolas-du-Pélem (Côtes
d'Armor) du Chalcolithique à la protohistoire ", Annales de Bretagne, 79 , p. 36-48.

Le Roux C.-T.

1974: Rapport scientifique sur la fouille de sauvetage du dolmen de Kercadoret en Moëlan-sur-Mer (Finistère), inédit, Rennes, SRA (Rapport, 1050), 4 p.

1975: " Informations archéologiques Bretagne ", Gallia Préhistoire, 18, p. 511-539.

1981: « Informations archéologiques Bretagne », Gallia Préhistoire, 24, p. $395-423$

1999: L'outillage de pierre polie en métadolérite du type $A$ : les ateliers de Plussulien (Côtes-d'Armor), production et diffusion au Néolithique dans la France de l'ouest et au-delà, Rennes, UMR 6566 « Civilisations atlantiques et Archéosciences », Université de Rennes 1 (Coll. Travaux du Laboratoire " Anthropologie, Préhistoire et Quaternaire Armoricain », 43), 244 p.

Le Rouzic Z.

1898: " Carnac, fouilles faites dans la région, en $1897 \&$ Janvier 1898 », BSPM, p. 64-80.

1930: "Bijoux en or découverts dans les dolmens du Morbihan », Revue des Musées, 5, p. 169-175.

1965 (†): Inventaire des monuments mégalithiques de la région de Carnac. L'arrondissement de Lorient, BSPM 92, p. 3-88.

Lisle du Dréneuc P. (De)

1892: « Notice sur les fouilles du tumulus de la Motte, Sainte-Marie (LoireInférieure) », BSANDLI, 31, p. 199-203.

LUKIS J. W.

1846: « Proceedings of the Association, May $12 », J B A A, 3$, p. 126.

Magny L. (DE)

1856: Nobiliaire universel : recueil général des généalogies historiques et véridiques des maisons nobles de l'Europe, Paris, Institut héraldique, 303 p.

MÉrimée P.

1837: Notes d'un voyage dans l'ouest de la France, Bruxelles, Société belge de Librairie, 438 p.

Micault V.

1881: " Essai sur la détermination de l'âge de quelques tumuli de Bretagne ", $B S E C N, 19$, p. 121-163.

1883: «Épées et poignards de bronze des Côtes-du-Nord, Finistère et Ille-etVilaine ", BSECN, 21, p. 71-123.

Mohen J.-P., Berger M.-T., Bouron M. Buisson D., Louboutin C., Eluère C. Duval A., Beck F., Chew H., Vallet F., Cleyet-Merle J.-J., Brière J.

1994: Musée des antiquités nationales, 
Saint-Germain-en-Laye, Guide, éd. revue et corrigée, Paris, Réunion des Musées nationaux, 262 p.

Mortillet A. (DE)

1902: « L'or en France aux temps préhistoriques et protohistoriques ", REAP, 12 , p. 47-72.

1903 : «L'argent aux temps préhistoriques en Europe », REAP, 13, p. 1-24.

\section{NeEdham $S$.}

2000a: Power pulses across a cultural divide : cosmologically driven acquisition between Armorica and Wessex, Proceedings of the prehistoric Society, 66, p. 151-207.

2000b: « The Gold and Copper Metalwork », in Hughes G., The Lockington Gold Hoard : an Early Bronze Age Barrow Cemetery at Lockington, Leicestershire, Oxford, Oxbow Books, p. 23-47.

2005: " Transforming Beaker Culture in North-West Europe; Processes of Fusion and Fission ", Proceedings of the prehistoric Society, 71, p. 171-217.

Needham S., Woodward A.

2008: "The Clandon Barrow Finery : a Synopsis of Success in an Early Bronze Age World ", Proceedings of the prehistoric Society, 74, p. 1-52.

Nicolas C.

2011: «Artisanats spécialisés et inégalités sociales à l'aube de la métallurgie : les pointes de flèches de type armoricain dans le nord du Finistère ", $B S P F, 108$, p. 93-125.

2012: «Des pierres précieuses? Les pointes de flèches du Campaniforme et de l'Âge du Bronze ancien en Bretagne (2500-1700 avant notre ère) », in Marchand G., Querré G. (Dir.), Roches et société de la Préhistoire entre massifs cristallins et bassins sédimentaires, Rennes, Presses universitaires de Rennes (Coll. Archéologie et Culture), p. 205-219.

Nicolas C., Stevenin C., Stephan P.

À paraître: "L'artisanat à l'âge du Bronze ancien en Bretagne ", in BouludGazo S., Nicolas T. (DIr.), Artisanats et productions à l'âge du Bronze. Approches techniques et sociales, contextes de découvertes, Actes de la Journée de la Société préhistorique française, 8 octobre 2011, Nantes, Paris, SPF.

Pailler Y., Gandois H., Ihuel E., Nicolas C., Sparfel Y.

2010: « Le bâtiment en pierres sèches de Beg ar Loued, Ile Molène (Finistère) : évolution d'une construction en pierres sèches du Campaniforme au Bronze ancient ", in Billard C., Legris M. (DIR.), Premiers Néolithiques de l'Ouest : Cultures, réseaux, échanges des premières sociétés néolithiques à leur expansion, Rennes, Presses universitaires de Rennes (Coll. Archéologie et Culture), p. 415-440.

Papillon F.

1997: Contribution à l'étude de la paléométallurgie du cuivre et du cuivre-arsenic à partir de l'analyse des éléments légers et de fusions expérimentales, thèse de doctorat, université de Paris $\mathrm{XI}, 200 \mathrm{p}$.

Paulet D.

2005: «Talutages en Forêt de Carnoët : premières investigations ", BSHAPL, 3, p. 109-114.

Pétrequin P., Errera M., Rossy M, aVec la Collaboration de D'Amico C., GHEDINI M.

2012: «Viso ou Beigua : approche pétrographique du référentiel des "jades alpins" ", in Pétrequin P., CASSEN S., Errera M., Klassen L., Sheridan A., Pétrequin A.-M. (DIR.), Jade. Grandes haches alpines du Néolithique européen. $V^{e}$ au IVe millénaires av. $J .-C$, Besançon, Presses universitaires de Franche-Comté (Coll. Les Cahiers de la MSHE Ledoux), p. 292-419.

Piggot S.

1938: " The Early Bronze Age in Wessex », Proceedings of the prehistoric Society, 4 , p. 52-106.

Prieto Martinez M. P., Salanova L.

2009: «Coquilles et Campaniforme en Galice et en Bretagne : mécanismes de circulation et stratégies identitaires ", $B S P F, 106$, p. 73-94.

Quilliec B.

2007: L'épée atlantique : échanges et prestiges au Bronze final, Paris, SPF (Coll. Mémoire de la SPF, 42), 171 p.

ReInaCh S.

1898: Catalogue sommaire du Musée des Antiquités Nationales au Château de Saint-Germain-en-Laye, $3^{\text {e }}$ édition, Paris, Librairies-Imprimeries réunies, $258 \mathrm{p}$.

1917: Catalogue illustré du Musée des Antiquités nationales au Château de Saint-Germain-en-Laye, Paris, Ernest Leroux, t. 1, 264 p.

REVUe ARCHÉOLOGIQUe

1844: "Dons faits au Musée de Cluny », Revue archéologique, 1, p. 132-134.

Riquet R., Guilaine J., Coffyn A.

1963: « Les campaniformes français (état actuel des recherches et perspectives) $»$, Gallia Préhistoire, 6, p. 63-128.
Rogers E. H.

1947: " The excavation of a barrow on Brownstone Farm, Kingswear ", Proceedings of the Devon archaeological Exploration Society, 3, p. 164-166.

Roussot-Laroque J.

2008: «Un inconnu célèbre : le poignard décoré du Bronze ancien de Sauliacsur-Célé (Lot) », PSO, 14, p. 149-160.

Salanova L.

1996: Des Cloches et des coquillages... fabrication et ornementation des vases campaniformes en France, thèse de doctorat, université de Paris 1, 3 vol., $1009 \mathrm{p}$.

2000: La question du Campaniforme en France et dans les îles anglo-normandes : Productions, chronologie et rôles d'un standard céramique, Paris, éd. Comité des Travaux historiques et scientifiques, $391 \mathrm{p}$.

2003: «Les sépultures mégalithiques et le phénomène campaniforme ", in Gonçalves V. S. (ÉD.), Muita gente, poucas antas? Origens, espaços e contextos do megalitismo, actas do 2 Colóquoi internacional sobre megalitismo, Reguengos de Monsaraz, 3 a 7 de Maio de 2000, Lisboa, Instituto portuguès de arqueologia, p. 385-393.

2011: " Chronologie et facteurs d'évolution des sépultures individuelles campaniformes dans le nord de la France ", in SAlanova L., TchérémissinofF Y. (DIR.), Les sépultures individuelles campaniformes en France, Paris, CNRS Éditions (Suppl. à Gallia Préhistoire, 41), p. 125-142.

Salanova L., Renard C., Mille B.

2011: «Réexamen du mobilier de la sépulture campaniforme d'Arenberg, Wallers (Nord) ", in SALanova L., TCHÉRÉMISSINOFF Y. (DIR.), Les sépultures individuelles campaniformes en France, Paris, CNRS Éditions (Suppl. à Gallia Préhistoire, 41), p. 79-95.

SANGmeister E.

1964: « Die schmalen “Armschutzplatten” », Studien aus Alteuropa, 1, p. 93-122.

SChwenzer S.

2009: «Vollgriffdolche der frühen Bronzezeit in der Schweiz : Statussymbole und Zeugnisse gusstechnischen Könnens », Helvetia archaeologica, 159/160, p. 66-96.

Serret A.

1885: Catalogue du musée archéologique et du musée des anciens costumes bretons de la Ville de Quimper, Quimper, Typ. Caen, $178 \mathrm{p}$.

1901: Catalogue du musée archéologique et du musée des anciens costumes bretons de la Ville de Quimper, $2^{\mathrm{e}}$ édition, Quimper, Imprimerie Cotonnec, 138 p. 
Sheridan A.

2007: "Scottish Beakers dates: the good, the bad and the ugly ", in LARSSON M., Parker Pearson M. (Dir.), From Stonehenge to the Baltic. Living with cultural diversity in the third millennium $B C$, Oxford, BAR (International Series, 1692), p. 91-123.

\section{Sheridan A., Pailler Y.}

2012: «Les haches alpines et leurs imitations en Grande-Bretagne, dans l'île de Man, en Irlande et dans les Iles anglo-normandes », in Pétrequin P., CAsSen S., Errera M., Klassen L., Sheridan A., Pétrequin A.-M. (DIR.), Jade. Grandes haches alpines $d u$ Néolithique européen. Ve au IVe millénaires av. $J .-C$., Besançon, Presses universitaires de Franche-Comté (Coll. Les Cahiers de la MSHE Ledoux), p. 1046-1087.

Société d'Emulation des Côtes-du-Nord

1884: Trésors archéologiques de l'Armorique occidentale, Rennes-Saint-Brieuc, Leroy-F. Guyon, 8 p., 29 pl.

\section{SoEIro M. T.}

1982: « Esconderijo de Sequeade (Barcelos) », Arqueologia, 5, p. 62-67.

Sommerard E. (DU)

1847: Musée des Thermes et de l'Hôtel de Cluny : Catalogue et description des objets d'art de l'Antiquité, du Moyen-âge et de la Renaissance, exposés au musée, Paris, Hôtel de Cluny, $240 \mathrm{p}$.

1883: Musée des Thermes et de l'Hôtel de Cluny : Catalogue et description des objets d'art de l'Antiquité, du Moyen-âge et de la Renaissance, exposés au musée, Paris, Hôtel de Cluny, $692 \mathrm{p}$.

Sparfel Y., Pailler Y. (Dir).

2009: Les mégalithes de l'arrondissement de Brest, inventaire et essai de synthèse, Saint-Malo-Rennes, éd. CeRAA-Institut culturel de Bretagne (Coll. Patrimoine archéologique de Bretagne), $290 \mathrm{p}$.

Sparfel Y., Pailler Y.

2011: Pré-inventaire des monuments du Néolithique et de l'âge du Bronze dans l'arrondissement de Quimper, autorisation préfectorale $n^{\circ}$ 2010-209, rapport de prospection thématique, SRA Bretagne, Rennes, 3 vol.

Strahm C.

2007: " L'introduction de la métallurgie en Europe ", in GuIlaine J. (DIR.), Le Chalcolithique et la construction des inégalités. Tome 1: Le continent européen, Paris, Errance, p. 49-71

Taylor I., Nodier C., De Cailleux A.

1846: Voyages pittoresques et romantiques dans l'ancienne France: Bretagne, Paris, éd. Didot, 2 vol., 385 p.

Tinevez J.-Y.

1988: « La sépulture à entrée latérale de Beaumont en Saint-Laurent-surOust », RAO, 5, p. 55-78.

2007: «Le mobilier du nouveau cimetière », in LE Roux C.-T. (DIR.), Monuments mégalithiques à Locmariaquer (Morbihan). Le long tumulus d'Er Grah dans son environnement, Paris, CNRS éditions, (Suppl. à Gallia Préhistoire, 38), p. 153-161

Tomalin D. J.

1988: «Armorican Vases à Anses and their occurrence in southern Britain ", Proceedings of the prehistoric Society, 54, p. 203-221.

Treinen F.

1970: « Les poteries campaniformes en France », Gallia Préhistoire, 13, 1, p. 53-107 et 2, p. 263-332.

Voruz J.-L.

1996: " La chronologie absolue du Bronze Ancien "), in Mordant C., GaIfFE O. (DIR.), Cultures et Sociétés du Bronze Ancien en Europe, $117^{e}$ Congrès national des Sociétés historiques et scientifiques, Clermont-Ferrand, 1992, Paris, éd. CTHS, p. 97-164. 